\title{
Investigation of Rope Formation in Gas-Solid Flows using Flow Visualization and CFD Simulations
}

\author{
Venkata Subba Sai Satish Guda
}

Follow this and additional works at: https://researchrepository.wvu.edu/etd

\section{Recommended Citation}

Guda, Venkata Subba Sai Satish, "Investigation of Rope Formation in Gas-Solid Flows using Flow Visualization and CFD Simulations" (2017). Graduate Theses, Dissertations, and Problem Reports. 5720. https://researchrepository.wvu.edu/etd/5720

This Dissertation is protected by copyright and/or related rights. It has been brought to you by the The Research Repository @ WVU with permission from the rights-holder(s). You are free to use this Dissertation in any way that is permitted by the copyright and related rights legislation that applies to your use. For other uses you must obtain permission from the rights-holder(s) directly, unless additional rights are indicated by a Creative Commons license in the record and/ or on the work itself. This Dissertation has been accepted for inclusion in WVU Graduate Theses, Dissertations, and Problem Reports collection by an authorized administrator of The Research Repository @ WVU.

For more information, please contact researchrepository@mail.wvu.edu. 


\section{Investigation of Rope Formation in Gas-Solid Flows using Flow Visualization and CFD Simulations}

\section{Venkata Subba Sai Satish Guda}

Dissertation submitted to the

Benjamin M. Statler College of Engineering and Mineral Resources

at

\section{West Virginia University}

in partial fulfillment of the requirements for the degree of

Doctor of Philosophy

in

Department of Mechanical and Aerospace Engineering

Ismail Celik, Chair, $\mathrm{PhD}$

John Kuhlman, PhD

Wade Huebsch, $\mathrm{PhD}$

Vyacheslav Akkerman, $\mathrm{PhD}$

Madhava Syamlal, PhD

Department of Mechanical and Aerospace Engineering

Morgantown, West Virginia

August 2017

Keywords: Roping phenomenon, Clustering phenomenon, Vorticity 


\begin{abstract}
Investigation of Rope Formation in Gas-Solid Flows using Flow Visualization

and CFD Simulations
\end{abstract}

\title{
Venkata Subba Sai Satish Guda
}

Coal is still one of the widely-used resources for power generation all over the world. Most of the relevant industries use pulverized coal as fuel which is delivered to the furnace by pneumatic conveying. Extensive use of coal has resulted in severe environmental problems due to emissions such as Carbon dioxide, Nitrogen and Sulphur compounds among others. It is postulated that if combustion efficiency is improved, this will lead to significant reduction in pollutant emissions. Combustion efficiency of pulverized coal power plants is influenced strongly by particle size distribution. Most industries use Cyclone Separators (or Classifiers) to separate the larger particles from the smaller ones as part of pre-combustion processes. The sizing and scaling of these classifiers are mostly based on empirical formulations. Detailed 3D numerical studies of these classifiers have not been successful in prediction of experimental observations, hence as such cannot be used as reliable tools for scale up studies. The main reason for this anomaly is believed to be failure of the models in capturing the dynamics of particle behavior in bends and ducts where particles form rope like structures with dense particle clusters. It is then imperative that more study is needed into the understanding of rope or cluster formation in gas-solid flows.

The main objective of the current study is to investigate the underlying mechanisms of rope formation phenomena. Gas-solid flow experiments have been performed in a vertical- horizontal $90^{\circ}$ glass bend with high speed imaging of the rope formation. Also, several Computational Fluid Dynamics (CFD) simulations have been performed using the commercial CFD package Ansys FLUENT to capture the roping phenomenon, and results have well supported the experimental observations. Several factors affecting rope formation have also been studied. Roping is basically a type of particle clustering in the sense high particle concentration regions are formed in both these phenomenon. Simulations have been performed on Fluid bed risers to capture clustering phenomenon and also to study the role of vorticity in cluster and rope formation with an objective of developing a fundamental definition for roping. MFIX, a multiphase flow code developed by NETL has also been used to capture the roping phenomenon. These results showed that high particle concentration was found to be in low vorticity regions surrounded by clockwise and counter-clockwise vortices. It was observed that there is indeed a vortex roller effect behind the formation of ropes. These results can be used to provide direction in development of computational models to better handle the gas-solid flow dynamics in classifiers 


\section{ACKNOWLEDGEMENT}

First and foremost I would like to thank Dr. Ismail Celik for all the wonderful guidance, support and help without which this work wouldn't have been possible. He has been a wonderful mentor under whom I was able to work on several interesting topics and explore the world of CFD. He has always given me the drive to set goals and achieve them. He has always been an understanding and caring advisor in times of need and support.

Secondly my heartfelt thanks to Dr. Wade Huebsch who was my advisor in my Master's program. It was under him that I learnt a lot about advanced aspects of fluid flow and also as part of his research group a lot about commercial CFD softwares. It was only with his help that I could start working on my $\mathrm{PhD}$ program.

I would like to thank Dr. Kuhlman for the wonderful guidance and knowledge transfer right from my second semester when I took the course "Fundamentals of Turbulent Flow" under him. It was with his help and support in times of need that I could successfully finish my PhD program.

I would like to thank Dr. Vyacheslav Akkerman and Dr. Madhava Syamlal, experts in Fluid and Multiphase Flows, for agreeing to be my committee members and providing their wonderful suggestions and guidance for this study.

I would like to thank Dr. Steve Rowan and Dr. Tao Yang who have provided me with great help and guidance in this study. I can never forget the hours spent with Dr. Steve in setting up and running various experiments and the wonderful conversations we had.

I would like to thank Mr. Frank Shaffer for helping me a lot with the Flow visualization part of this study which has been the backbone for validation in our study. 
I would like to thank all my professors at West Virginia University for the wonderful knowledge they have transferred to me. They have been great teachers.

I would like to thank the entire MAE Department staff for all their help in making my program go smoothly.

I would like to thank all my group members Sergio, Jose, Hayri, Albio, Hunter, Mehmet, Sharad, Gennaro, Chris Griffin and Nihan who have always helped me and created a family like work environment which made me comfortable at work. Along with a degree, I got friends for life.

My sincere thanks to Dr. Raju Pakalapati for helping me to enter this wonderful research group and all the support at the beginning stages.

$\mathrm{PhD}$ program is never easy. The problems we face, the hours of struggle to solve problems and work pressure are all the side aspects of this. The only solution to all these is the Smile on our face. I would like to thank all my friends at Morgantown who have always been there for me and made sure that life keeps going smoothly and happily and helped me accomplish my goal.

I can never express in words my gratitude and love for my parents and family. They have always been a rock solid support for me. They have guided, supported and motivated me a lot in pursuing my goals. Without their love and prayers, this wouldn't have been possible.

My heartfelt thanks and salutations to my grandfather Dr. K. Sivananda Murthy garu for directing me, guiding me and making me achieve my $\mathrm{PhD}$ goal. He has always provided solutions to all the problems in such a manner that the problem never existed or reappeared later. Without him, this goal of $\mathrm{PhD}$ would have always remained a dream. Of course there is no question of him not being there for me. This degree is dedicated to him. 


\section{Contents}

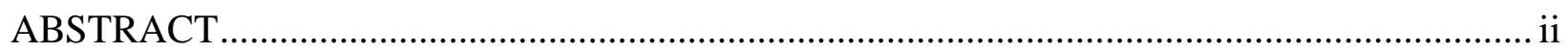

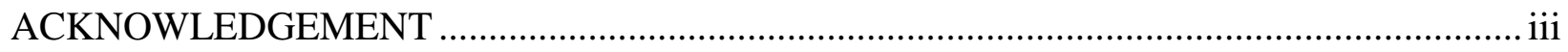

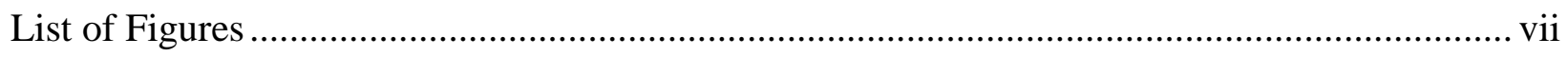

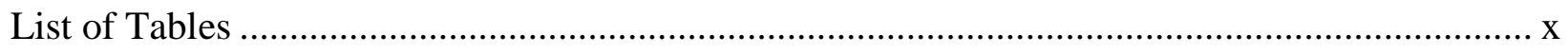

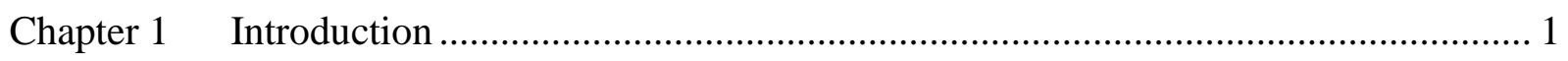

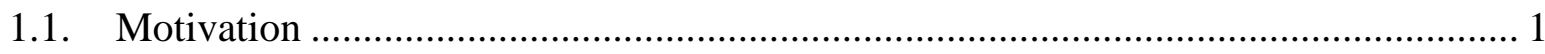

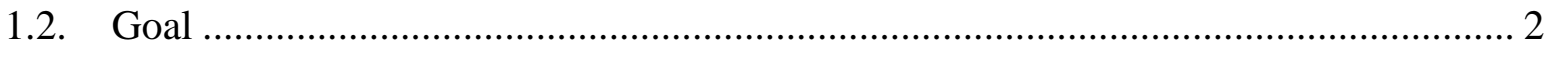

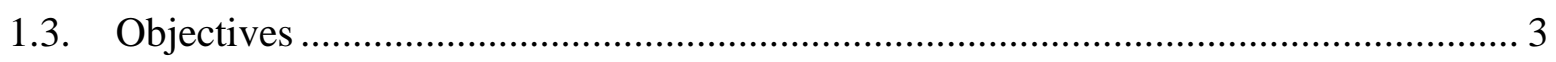

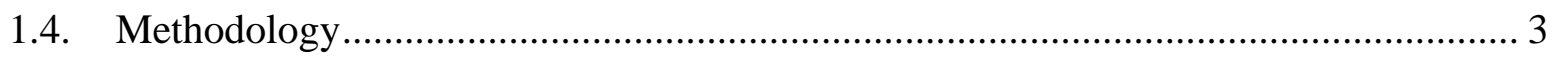

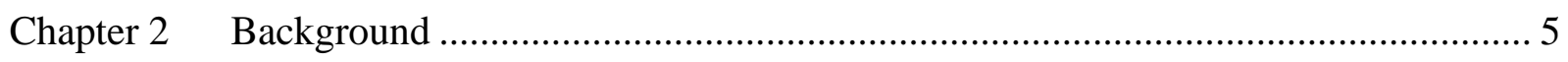

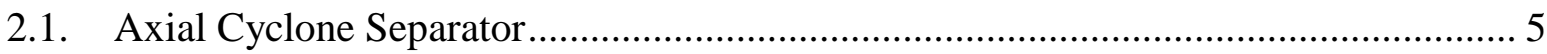

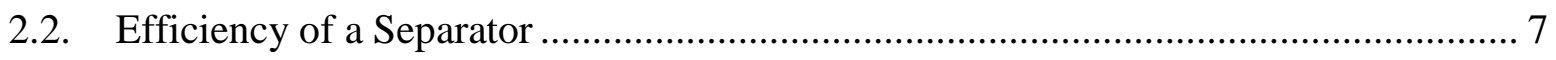

2.3. Numerical Simulations of Axial Cyclone Separator ……........................................ 8

2.4. Problems in Prediction of Cyclone Efficiency …………….................................... 11

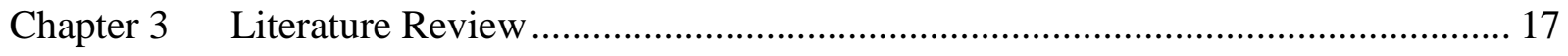

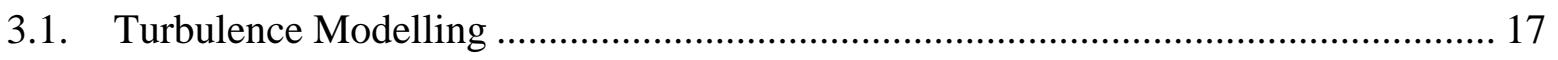

3.2. Numerical Modelling of Gas-Solid Flows................................................................. 21

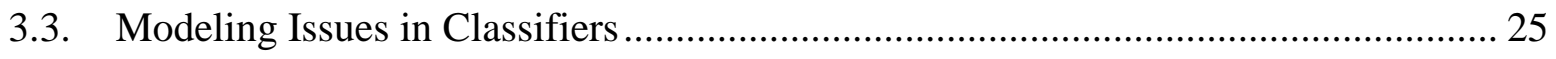

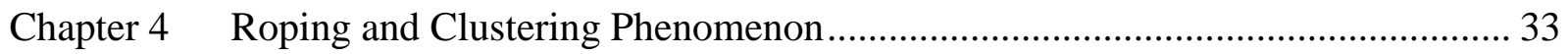




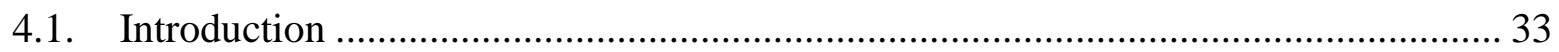

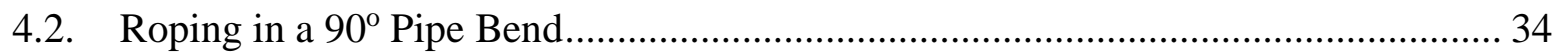

4.2.1. Experimental Study of Gas-Solid Flow in Pipe Bend ..................................... 35

4.2.2. Numerical Study of Gas-Solid Flow in Pipe Bend ...................................... 47

4.2.3. Comparison of Experimental and Numerical Results .................................. 55

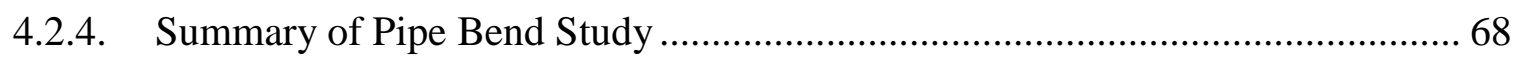

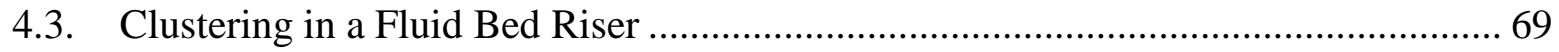

4.3.1. 2D Simulations using Ansys FLUENT ….............................................. 70

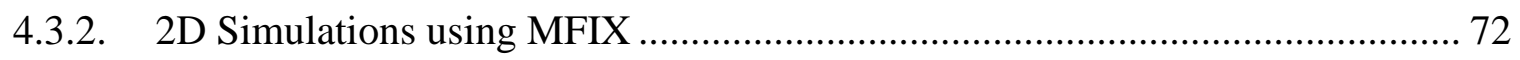

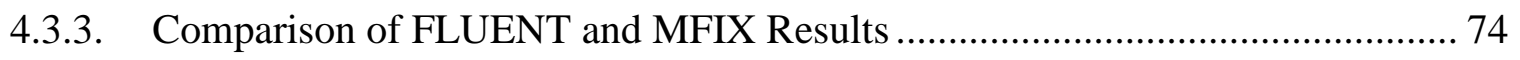

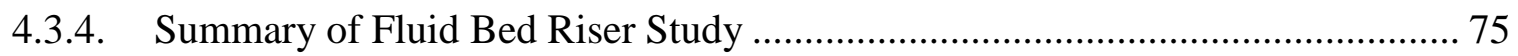

Chapter 5 Vorticity and Turbulence Kinetic Energy Effect ......................................... 76

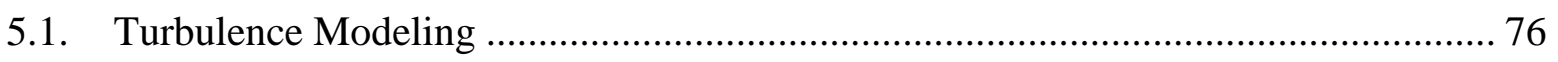

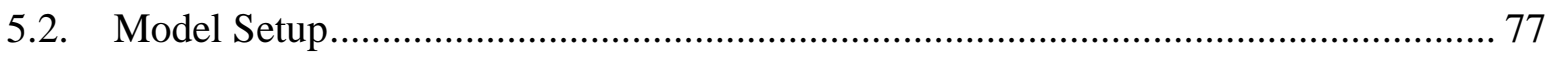

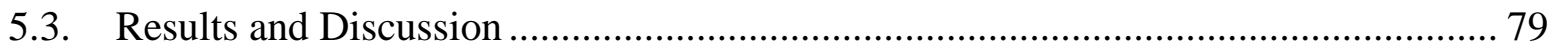

5.4. Summary of the Vorticity and T.K.E Study ...................................................... 92

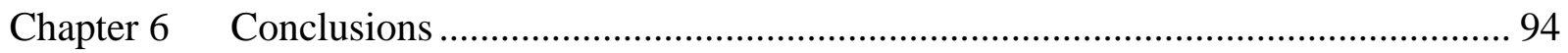

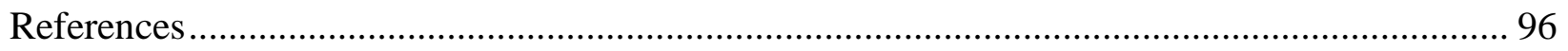

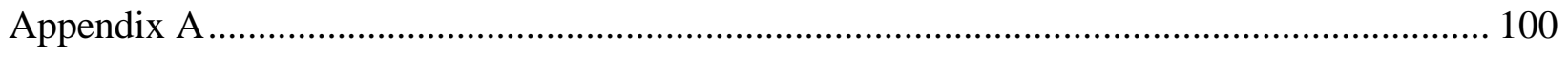




\section{List of Figures}

Figure 2.1 Axial cyclone separator .............................................................................. 5

Figure 2.2 Axial cyclone separator with particle tracks colored by particle diameter ................. 6 Figure 2.3 Results of axial cyclone separator with 60 degree vanes and particles modeled by RANS simulation compared with experiments a) trapped conditional probability, b) escaped conditional probability, c) axial gas velocity profiles, d) tangential gas velocity profiles.......... 10 Figure 2.4 Grid sensitivity study results a) trapped conditional probability, b) escaped conditional probability, axial gas velocity profiles - c) unrefined grid d) refined grid respectively; tangential gas velocity profiles - e) unrefined grid f) refined grid respectively 12 Figure 2.5 RANS vs LES results: axial gas velocity profiles - a) RANS c) LES respectively; tangential velocity profiles - b) RANS d) LES respectively; e) trapped conditional probability, f) escaped conditional probability

Figure 2.6 High particle concentration regions or ropes at the exit plane of the vane set in an

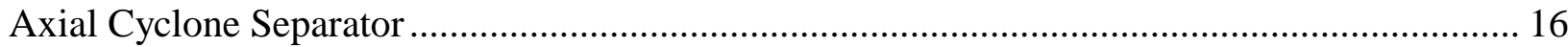

Figure 3.1 Visible rope in a horizontal pipe after a $90^{\circ}$ bend exit ......................................... 25

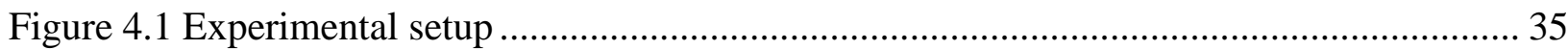

Figure 4.2 Snapshot of HDPE particles for a loading of $100 \%$................................................ 37

Figure 4.3 Snapshot of rope with flaxseed at loading of $42 \%$................................................... 37

Figure 4.4 Cumulative particle size and particle size density distributions for ground flaxseed particles (Courtesy of Mr. Jonathan Tucker, U.S. DOE NETL) ............................................. 38

Figure 4.5 Schematic of camera perspectives for high speed imaging ..................................... 40

Figure 4.6 Camera 1's view aligned with elbow exit ......................................................... 40

Figure 4.7 Camera 2's view of elbow exit from below .................................................... 41

Figure 4.8 Camera 3's view of elbow exit from the side .................................................. 41

Figure 4.9 Video frame from original high speed video showing particle flow field exiting the

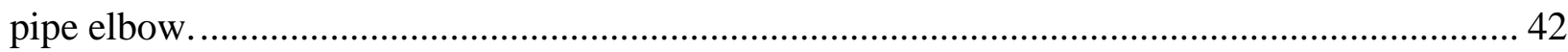

Figure 4.10 After a bandpass FFT was applied with a cutoff set at 30 pixels ............................ 43

Figure 4.11 After subtracting the background image ............................................................. 43

Figure 4.12 After applying an Otsu threshold filter............................................................. 43

Figure 4.13 Location of maximum brightness/concentration ............................................... 44

Figure 4.14 Locations downstream of elbow exit where measurements have been done ........... 44

Figure 4.15 Significant portion of flow field from side view .............................................. 46

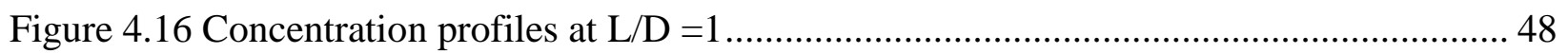

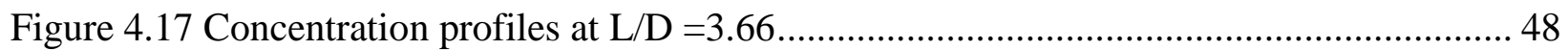

Figure 4.18 3-D coarse grid for simulations with cross-sectional view on the side .................. 49

Figure 4.19 Comparison of concentrations for different methods of loading at $\mathrm{L} / \mathrm{D}=0.25 \ldots \ldots . .50$ Figure 4.20 Comparison of concentrations for different inlet turbulent length scales at loading of $42 \%$ 
Figure 4.21 Comparison of concentrations for different inlet turbulent intensities at loading of $42 \%$

Figure 4.22 Comparison of concentrations for different particle size distributions and wall coefficients of restitution at loading of $35 \%$.................................................................. 53

Figure 4.23 Particle visualization from side view for all cases in Table 4.3 ........................... 57

Figure 4.24 Flow visualization from side view for $42 \%$ loading ............................................. 58

Figure 4.25 Concentration profiles for all cases at different locations for a loading of $42 \%$..... 59

Figure 4.26 Concentration profiles for all cases at different locations for a loading of $35 \%$...... 60

Figure 4.27 Particle concentration contour at different cross sections from LES simulations for $42 \%$ loading condition.

Figure 4.28 Contour plots of axial gas velocity from different views and different locations for LES simulation with $42 \%$ loading. 62

Figure 4.29 Contour plots of axial gas velocity from different views and different locations (RR)

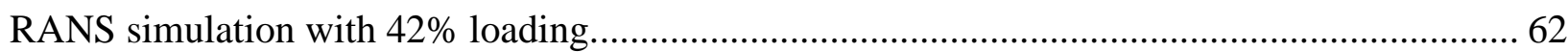

Figure 4.30 Definition of parameter $\lambda$ in the study of restitution coefficients..... 63 Figure 4.31 Particle concentration profiles from experiments and the RANS simulations for $42 \%$ loading condition with various values of $\lambda$. 64

Figure 4.32 Particle visualization figures from the side view for RANS simulations for $42 \%$

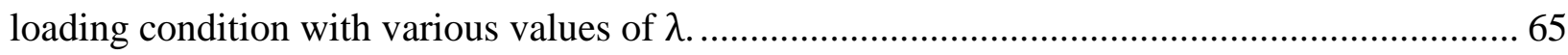

Figure 4.33 Concentrations at different locations for loading of $42 \%$ with $\lambda=1.3 \ldots \ldots \ldots \ldots \ldots \ldots . . . . . .66$

Figure 4.34 Particle visualization from side view for loading of $42 \%$ with $\lambda=1.3 \ldots \ldots \ldots \ldots \ldots \ldots .67$

Figure 4.35 Particle concentration contour at different cross sections from RANS (top) and LES

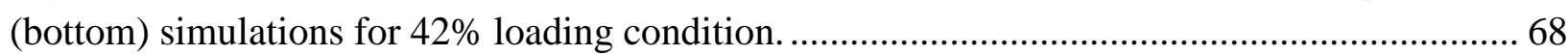

Figure 4.36 Contour plots of solids fraction for several grids with uniform inlet case with

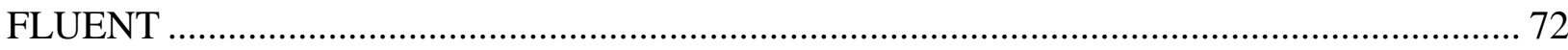

Figure 4.37 Contour plots of solids fraction for several grids with uniform inlet case with MFIX

Figure 4.38 MFIX and FLUENT showing particle clusters .................................................... 75

Figure 5.1 VLES Grid ................................................................................................... 78

Figure 5.2 Cross-sections at height a) $40 \mathrm{~cm} \mathrm{b)} 50 \mathrm{~cm}$ showing normalized particle concentration Cnorm $=$ C-0.2/0.05 overlapped by normalized Y-vorticity $(\mathrm{w} / \mathrm{s})$ in direction of flow ............ 80

Figure 5.3 Detailed analysis of a cluster location .............................................................. 82

Figure 5.4 Vertical cross-section with actual concentration with overlapped Z-vorticity........... 82

Figure 5.5 Roller effect of vortices in cluster or rope formation ........................................... 83

Figure 5.6 Comparison of clusters in cases 1, 2 and 3 respectively shown here are instantaneous

Figure 5.7 Plots of area-weighted average vorticity magnitude along time at various cross-

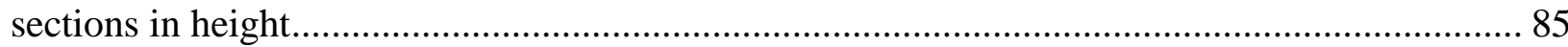

Figure 5.8 Contours of concentration overlapped by vorticity magnitude (top row) and Y-

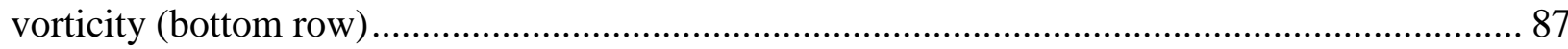

Figure 5.9 2D Fluid bed simulation with MFIX: Concentration vs Vorticity .......................... 88 
Figure 5.10 2D Fluid bed simulation with MFIX: Concentration vs T.K.E ............................. 89

Figure 5.11 Contours of variables at location downstream of pipe bend ............................... 91

Figure 5.12 Map summarizing formation of ropes ......................................................... 93 


\section{List of Tables}

Table 2.1 List of all simulations of cyclone with 60 degree vane set .................................... 9

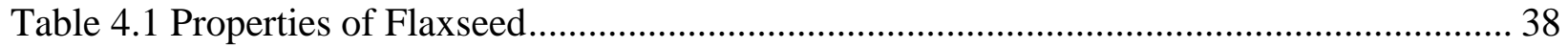

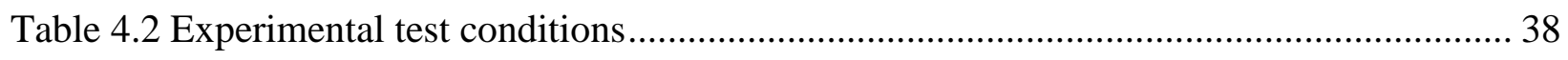

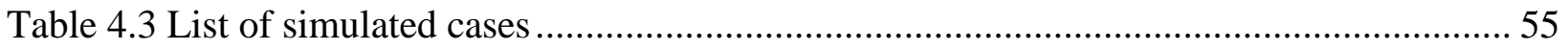

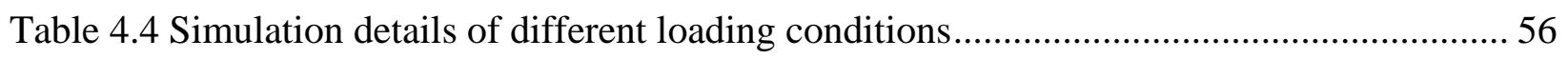

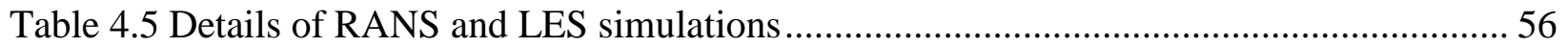

Table 5.1 Operating Conditions for all simulations in this study .......................................... 79 


\section{Chapter 1 Introduction}

\subsection{Motivation}

Coal is one of the widely used sources for energy production. It is a cheap and non-renewable resource. Coal has a wide variety of applications, the primary being energy production with its use as fuel. Thermal power plants have been primary means of power generation in many countries. They use pulverized coal to fire the furnaces. The traditional boiler system has been used in these plants for decades. The heat released as a result of combustion is used to convert water to steam which drives a turbine connected to generator thereby producing electricity. Not only coal, even its byproducts of combustion have several uses [1], [2]. Coal is also used popularly used in manufacturing of steel, hydrocarbons, liquid fuels and calcium carbide [3].

The wide usage of coal in several applications has caused a detrimental impact on the environment. Coal combustion has resulted in emissions of several pollutants such as $\mathrm{CO}_{2}, \mathrm{NO}_{\mathrm{x}}$ and $\mathrm{SO}_{\mathrm{x}}$ [4] which resulted in global warming, change in weather patterns, acid rain and major human health concerns [5]. For instance in thermal power plants, these problems of pollutants majorly arise due to incomplete and inefficient combustion of coal particles. Large coal particles do not burn completely and result in formation of gaseous pollutants like $\mathrm{CO}$ which are harmful to the health and also environment. These larger particles also result in combustion inefficiency resulting in only partial usage of the potential of a valuable fuel. Hence in order to prevent these problems, industries use Cyclone Separators or Classifiers to separate the large particles from the fine ones as part of pre-combustion processes. The coarser large particles are sent back to the mill for further crushing. These have abrupt turns in direction of flow by employing vane sets or bends in order to classify particles by inertial effects. 
Before installation of these classifiers at a full scale level, prototypes or lab scale models are designed and tested. Also several experiments and numerical studies have been performed in order to develop classifiers with high efficiency of separation. A common problem of most of these studies was inconsistencies between the experimental and numerical results. The modelling results differed greatly from experimental results. These discrepancies have cost industry time and money as the existing models cannot be used reliably for scale up studies. It was observed in simulations in this study that most of the particles failed to leave the computational domain. The major reason for the anomaly is the dynamics of gas-solid flow behavior in bends and ducts. Regions of high particle concentrations known as 'ropes' are formed. This phenomenon is known as 'roping'. This phenomenon is similar to particle clustering in the sense that high particle concentration regions are formed in both these phenomenon. This phenomenon is found to be the primary reason for the inconsistencies between experimental and simulation results of the studies of cyclone separators or classifiers. In order to develop suitable models to effectively and accurately model the cyclone separators or classifiers, understanding the physics of roping phenomenon is very pivotal.

\subsection{Goal}

The main purpose of this work is to understand the physics behind the formation of ropes or roping phenomenon in gas solid flows. Based on this, a fundamental definition of a rope will be formulated. The proposed definition and the inferences from this study can be used in the development of CFD models that will take into account this phenomenon and accurately predict the functioning of a cyclone separator or classifier. 


\subsection{Objectives}

The physics behind roping phenomenon is being investigated. So accomplishing certain objectives in a certain order is necessary to rightly understand the nature of the dynamics of these gas-solid flows. The main objectives for this study are:

- Developing an accurate gas-solids flow model using Ansys FLUENT to predict roping phenomenon with model validation based on experimental data.

- High particle concentration regions are a common aspect of both roping and clustering. Hence modelling fluid bed risers to see if similar phenomenon occur which would help to understand flow physics involved in roping.

- Capturing roping phenomenon with the code MFIX, which is state of the art method for numerically modelling two-phase flows with high solids concentrations.

- Study effects of factors like vorticity and kinetic energy on roping. These might likely be of help in better understanding the physics involved in this phenomenon and might be instrumental in the development of computational models to accurately capture the functioning of separators.

\subsection{Methodology}

The main methodology involved using the commercial CFD package Ansys FLUENT to capture the roping phenomenon for gas-solid flows in a simple geometry such as a 90 degree pipe bend. For this, the Eulerian-Lagrangian Discrete Phase Model (DPM) has been used. The gas phase was handled by the Eulerian approach and the particles by the Lagrangian framework. The interaction between the two phases is by means of source terms that couple the governing equations. 
The first step involved validating the CFD model by reproducing past numerical studies. The next step has been to successfully capture roping in experiments and then replicate this in simulations. This also involved studying various new factors that might affect rope formation. The next step has been to study the role of gas-dynamics in the formation high particle concentration clusters in fluid bed risers. The role of several factors influencing this behavior in fluid bed risers has been investigated. This phenomenon in fluid bed risers has also been investigated using MFIX, a numerical code developed by NETL, which is widely used to handle flows with high particle concentration. These results have been compared to Ansys results. Based on these results, the properties affecting roping have been studied, the physics behind rope formation were investigated and possible suggestions were detailed out to be implemented in fixing models to effectively capture the functioning of cyclone classifiers numerically. 


\section{Chapter 2 Background}

\subsection{Axial Cyclone Separator}

Cyclone separators are used to separate particles from conveying air by rotational, centrifugal or gravitational effects [6]. Some of the separators (also known as classifiers) are used to separate coarse particles from the finer ones. These are commonly used in saw mills, oil refineries, cement industries, food processing industries and simple home applications like vacuum cleaners. These are also used in thermal power plants to separate coarse coal particles from the fine ones in order to improve combustion efficiency and prevent pollutant emissions due to incomplete combustion. An axial cyclone separator is shown in the Figure 2.1below.

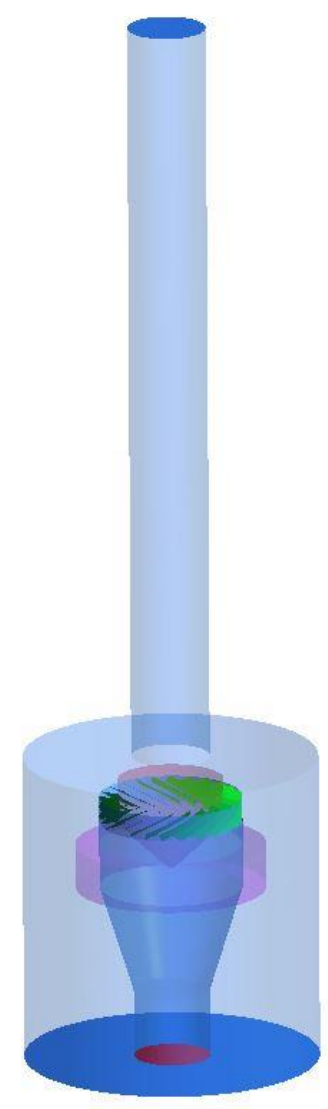

Figure 2.1 Axial cyclone separator 
The particles are carried by the air from the inlet (dark red) through the inner piping sections. They then pass through the vane section, which has vanes inclined at a specific angle. Due to the centrifugal, inertial and gravitational effects as a result of abrupt change in direction of flow, the coarser particles impact the walls and are collected at the bottom whereas the smaller particles follow the path of air and escape through the outlet of the long pipe at the top. In this way, the coarser particles are separated from the finer ones. The larger particles collected at the bottom and the finer particles that escape from the top are collected separately. Figure 2.2 below shows a simulation of particle flow through the cyclone with smaller particles escaping and large particles getting collected at the bottom.

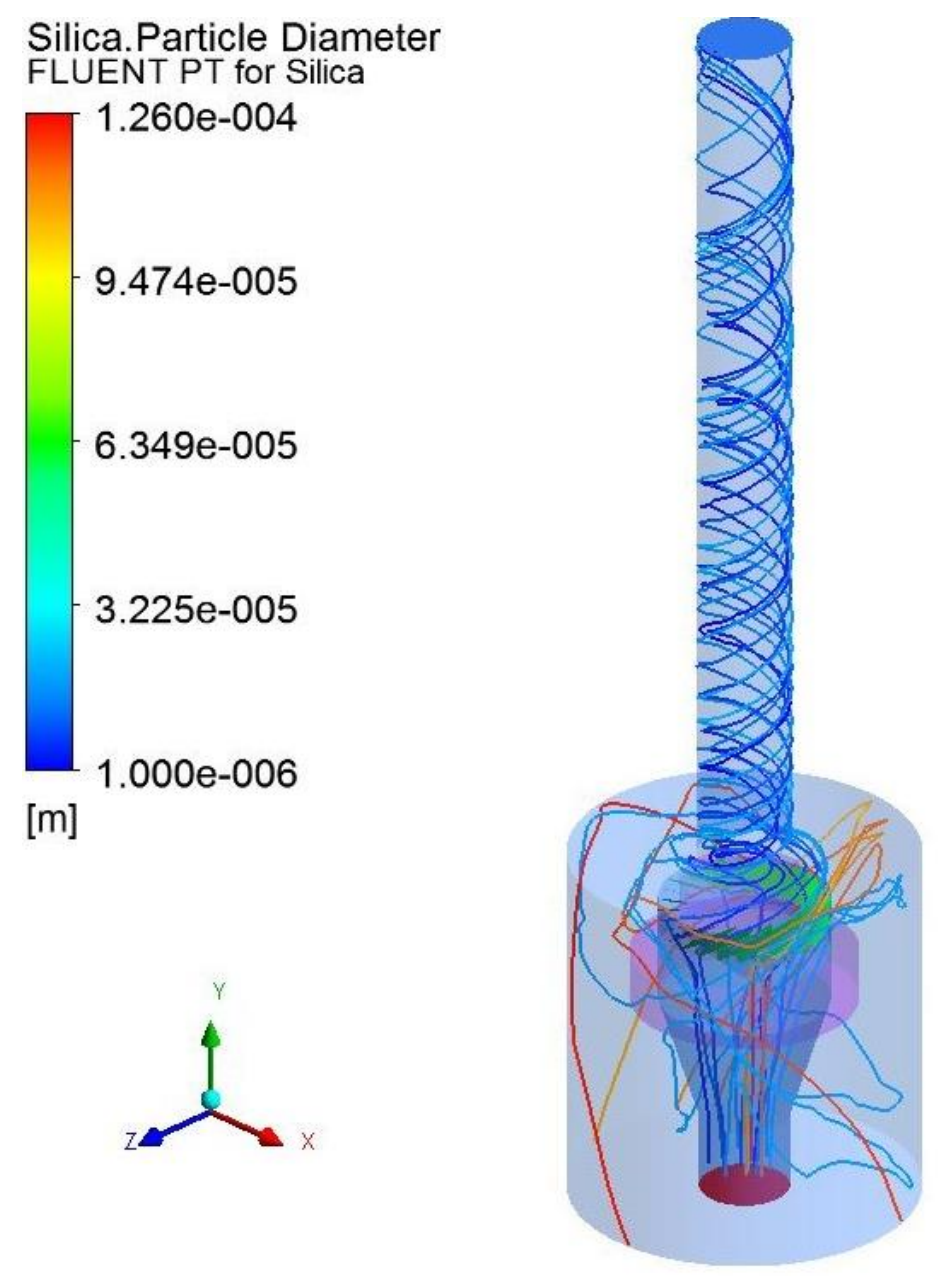

Figure 2.2 Axial cyclone separator with particle tracks colored by particle diameter 


\subsection{Efficiency of a Separator}

The efficiency of the cyclone separator or classifier is the ability to separate the coarse particles from the finer ones. Basic conditional probability concepts have been used in this initial study to compare experimental and numerical results of the separator.

Mass fraction of trapped particles is given by:

$$
\text { MF Trapped }=\frac{\text { Total mass trapped }}{\text { Total inlet mass }}
$$

Mass fraction of escaped particles is given by:

$$
\text { MF Escaped }=\frac{\text { Total mass escaped }}{\text { Total inlet mass }}
$$

Normalized mass fraction trapped of a size bin range (i) is given by:

$$
(\text { NMF Trapped })_{i}=\frac{\text { Mass trapped in range ' } i^{\prime}}{\text { Total mass trapped }}
$$

Normalized mass fraction escaped of a size bin range (i) is given by:

$$
(\text { NMF Escaped })_{i}=\frac{\text { Mass escaped in range ' } i}{\text { Total } \text { mass escaped }}
$$

Probability of a particle trapped in a size bin range (i) is given by:

$$
P_{\text {trapped }(i)}=\frac{(\text { NMF Trapped })_{i}}{\text { Size of bin range }{ }^{\prime} i^{\prime}}
$$

Probability of a particle escaped in a size bin range (i) is given by:

$$
P_{\text {escaped }(i)}=\frac{(N M F \text { Escaped })_{i}}{\text { Size of bin range }{ }^{\prime} i^{\prime}}
$$

where NMF is Normalized Mass Fraction. 


\subsection{Numerical Simulations of Axial Cyclone Separator}

Axial Cyclone Separator is a widely used industrial application whose scale up studies have been affected by abrupt turns in gas-solid flow direction. A numerical study of the functioning of an axial cyclone has been performed using the commercial CFD software Ansys Fluent. The materials used in these simulations are mainly silica and coal. The numerical results have been compared with the experimental results of LP AMINA researchers [46] for the same materials. The simulations have been performed for axial cyclones fitted with 60 degree and 30 degree vane sets respectively. The designs for the two vane sets are mostly the same with minor changes in the positions of the vane sets and associated bluff body. Transient simulations have been performed with steady particle tracking. The Discrete Phase Model has been used for handling the particles in a Lagrangian framework while gas is treated as a continuum. The injection of particles at the inlet has been done by a random method with no specific preference to size. Table 2.1 below shows the list of all simulations that have been performed. 
Table 2.1 List of all simulations of cyclone with 60 degree vane set

\begin{tabular}{|c|c|c|c|c|c|c|c|}
\hline S.No & $\begin{array}{c}\text { Diameter } \\
\text { range } \\
\text { (microns) }\end{array}$ & $\begin{array}{c}\text { Loading } \\
\text { (A/F) }\end{array}$ & $\begin{array}{l}\text { Shape } \\
\text { factor }\end{array}$ & $\begin{array}{c}\text { Coefficient } \\
\text { of } \\
\text { restitution }\end{array}$ & $\begin{array}{l}\text { Number of } \\
\text { trajectories }\end{array}$ & Turb. model & Material \\
\hline 1.1 & $1-210$ & $15: 1$ & 1 & 0.9 & 50000 & RNG k-e & Silica \\
\hline 1.2 & $1-210$ & $15: 1$ & 0.7 & 0.9 & 50000 & RNG k-e & Silica \\
\hline 1.3 & $1-210$ & $15: 1$ & 1 & 0.7 & 50000 & RNG k-e & Silica \\
\hline 1.4 & $1-210$ & $15: 1$ & 0.7 & 0.7 & 50000 & RNG k-e & Silica \\
\hline 1.5 & $\begin{array}{c}100-150 \\
\text { (mono) }\end{array}$ & $15: 1$ & 1 & 0.9 & 50000 & RNG k-e & Silica \\
\hline 1.6 & $\begin{array}{c}100-150 \\
\text { (mono) }\end{array}$ & $6: 1$ & 1 & 0.9 & 50000 & RNG k-e & Silica \\
\hline 1.7 & $1-210$ & $15: 1$ & 1 & 0.9 & 10000 & RNG k-e & Silica \\
\hline 1.8 & $1-210$ & $15: 1$ & 1 & 0.9 & 70000 & RNG k-e & Silica \\
\hline 1.9 & $1-210$ & $15: 1$ & 0.7 & 0.7 & 50000 & Mod RNG k-e & Silica \\
\hline 1.10 & $\begin{array}{c}1-210 \\
\text { (reconstructed) }\end{array}$ & $15: 1$ & 1 & 0.9 & 50000 & RNG k-e & Silica \\
\hline 1.11 & $1-210$ & $15: 1$ & 0.7 & 0.7 & 50000 & $\mathrm{k}-\mathrm{w}$ & Silica \\
\hline 1.12 & $\begin{array}{c}\text { 1-210 } \\
\text { (grid } \\
\text { sensitivity) }\end{array}$ & $15: 1$ & 1 & 0.9 & 50000 & $\mathrm{k}-\mathrm{w}$ & Silica \\
\hline 1.13 & $1-710$ & $15: 1$ & 1 & 0.9 & 50000 & RNG k-e & Coal \\
\hline
\end{tabular}

The results of the study case 1.4 are shown in Figure 2.3. The trapped and escaped conditional probabilities for different bin ranges and also the axial and tangential velocities at various locations are shown and compared with respective experimental data. This result summarizes the results of most of the above studies as there is hardly any variation in the trapped and escaped particle size 
distributions from case to case with change in parameters. The legends in the plots (c) and (d) below refer to lines drawn in the domain at different angles in radial direction on which velocity profiles have been obtained.

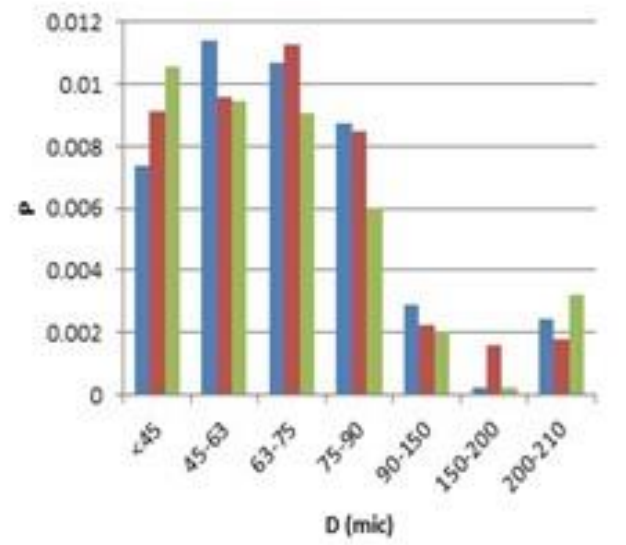

(a)

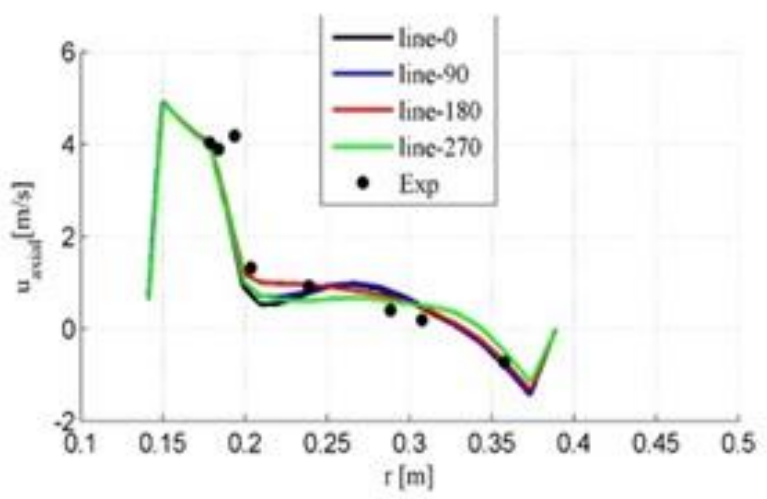

(c)

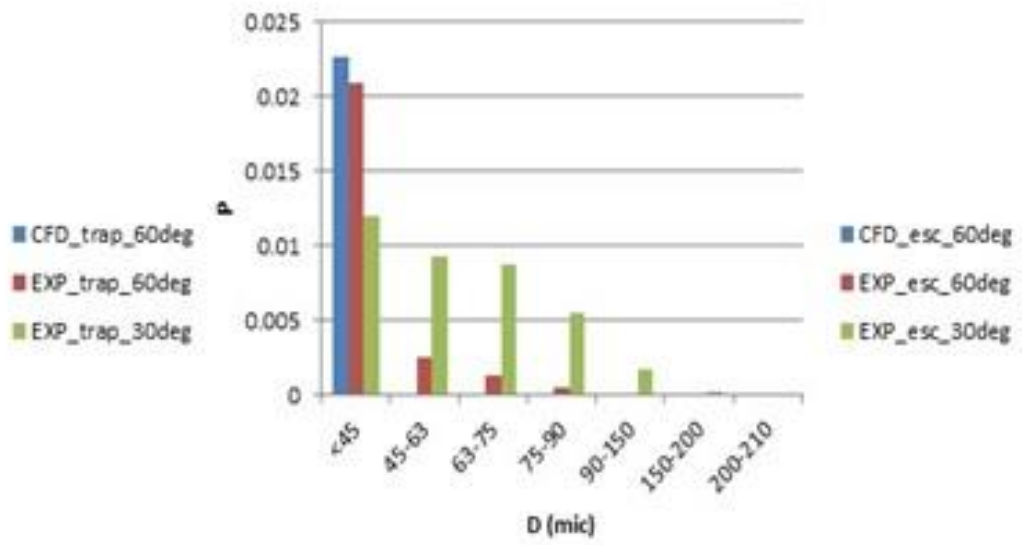

(b)

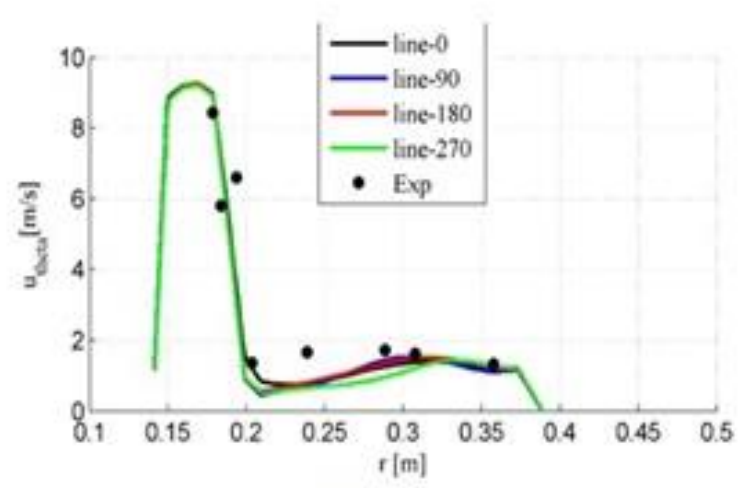

(d)

Figure 2.3 Results of axial cyclone separator with 60 degree vanes and particles modeled by RANS simulation compared with experiments a) trapped conditional probability, b) escaped conditional probability, c) axial gas velocity profiles, d) tangential gas velocity profiles.

It can be clearly seen from the trapped probability plot that there is significant difference in the probability values between experiments and CFD results. The difference is even more evident for the escaped particle probabilities. The CFD results under-predict in the smallest size bin range and over predict as the bin size increases. From the escaped probability plot, it can be seen that particles 
in the smallest bin range have much higher probability value than the experiments. A significant observation is that there are larger particles from other bin size ranges escaping in experiments. However the simulations don't show this behavior indicating an unrealistic high value of trapping efficiency. In reality, this did not occur in experiments. Such inconsistencies lead to doubts on the reliability of these models to lead scale-up studies. However the axial gas velocity profiles from simulations match well with experiments but the tangential gas velocity profiles are not predicted well.

\subsection{Problems in Prediction of Cyclone Efficiency}

From the above figure, clearly one can observe that there are significant differences between the experimental and computational results. There is some indication that the simulations are not able to predict the gas-solid flow behavior accurately. In order to find the reason behind this anomaly, several factors that might possibly affect the gas-solid flow behavior in simulations have been studied. Several properties like air to solids loading (A/F), diameter range, nature of diameter range (mono, reconstructed feed from trapped and escaped), different turbulence models, particle shape factors, number of trajectories and coefficients of restitution have been varied and studied. All of the results showed a significant difference between experimental and numerical findings. Glaring differences have been observed in the nature of escaped particles between simulations and experiments. Similar behavior was observed in simulations with 30 degree vane set too. Some possible causes are:

\section{a) Grid Coarseness}

The possibility of the grid being not fine enough and resulting in the possibility of inaccuracy in numerical results was considered. The grid was further refined and a grid sensitivity was performed. However there was very little change in results in the probabilities or the gas profiles. 
Figure 2.4 shows the grid sensitivity study results. Therefore the grid coarseness is not the reason behind the problem of concern.

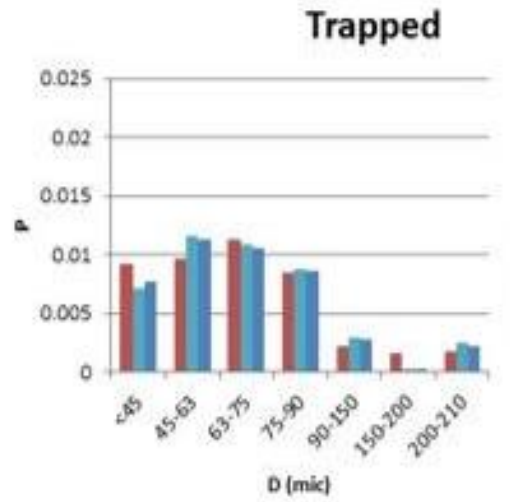

(a)

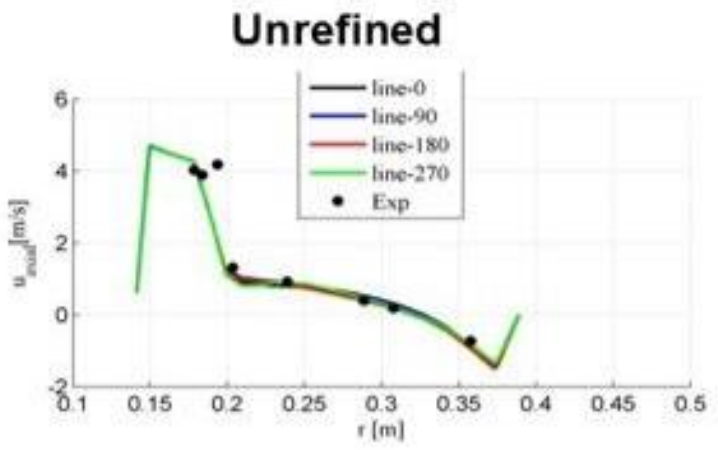

(c)

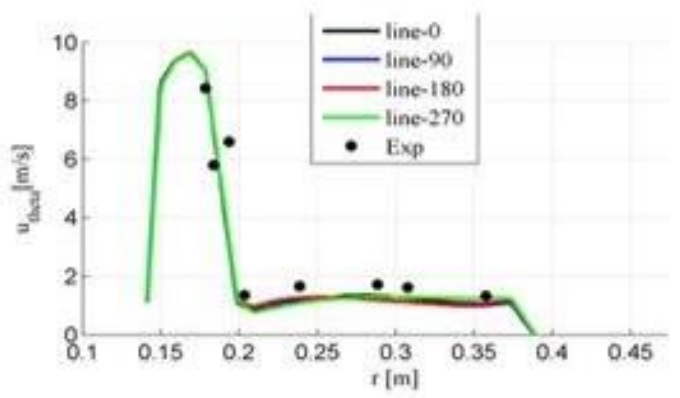

(e)

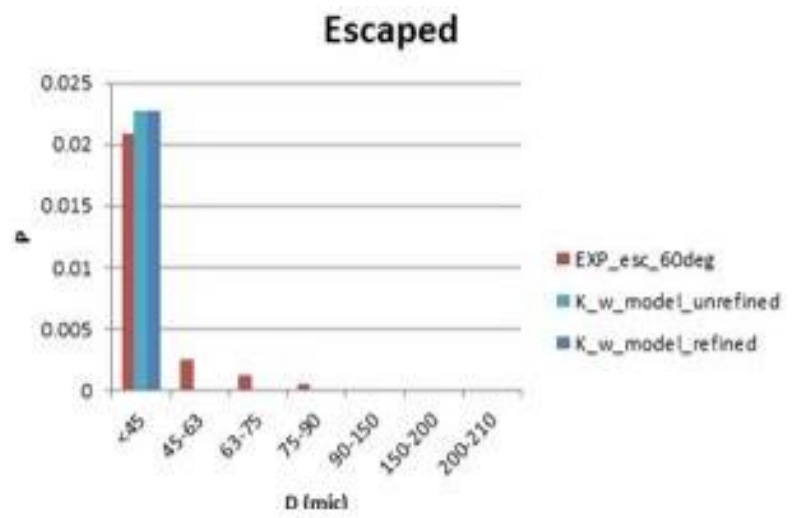

(b)

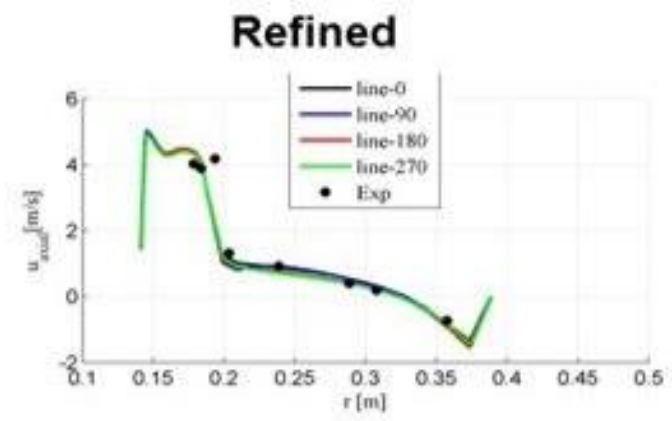

(d)

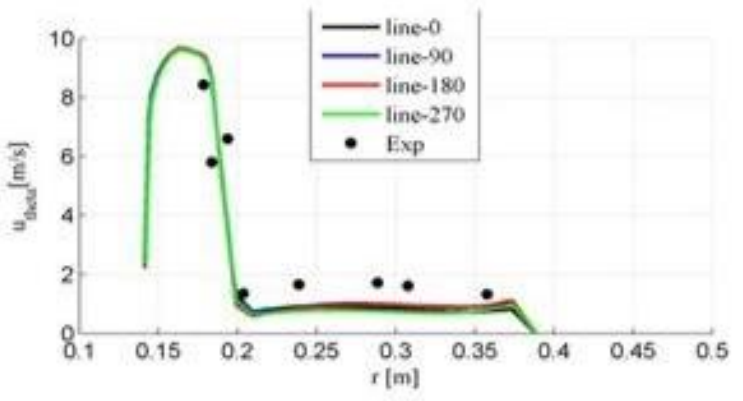

(f)

Figure 2.4 Grid sensitivity study results a) trapped conditional probability, b) escaped conditional probability, axial gas velocity profiles - c) unrefined grid d) refined grid respectively; tangential gas velocity profiles - e) unrefined grid f) refined grid respectively 


\section{b) Under-Prediction of Tangential Velocity}

In the functioning of the axial cyclone separators with vanes, the role of tangential velocity is very significant. The centrifugal forces rely more on the tangential velocity, based on which the separation of coarser particles from the finer ones occurs coupled with inertia effects. The common problem in all of the above simulations is the inaccurate prediction of tangential gas velocity. According to [7], large eddy simulations (LES) predicted tangential velocity much more accurately compared to RANS models. However for axial velocity profiles, no significant difference is found between the models. In all the above RANS cases, the axial gas velocity profiles matched well with the experiments. The shortcomings were only with the tangential velocity profiles. Hence a large eddy simulation has been performed on the axial cyclone to better capture the tangential velocity profiles and to see if there is any improvement in the escaped probabilities. Figure 2.5 below shows the results of the LES. It can be seen from a) and c) that axial velocity profiles are being captured accurately. The biggest improvement is in the tangential velocity profiles from b) to d). These profiles from LES are very close to experiments compared to RANS which are very inaccurate. The effect of this can be clearly seen in the probability plots of trapped and escaped particles. It can be observed from e) that the large under prediction of CFD results in the smallest size range of trapped particles is not that large in LES compared to RANS results. Also the next size range of particles showed up in the escaped plots which were totally absent in the RANS simulations. 


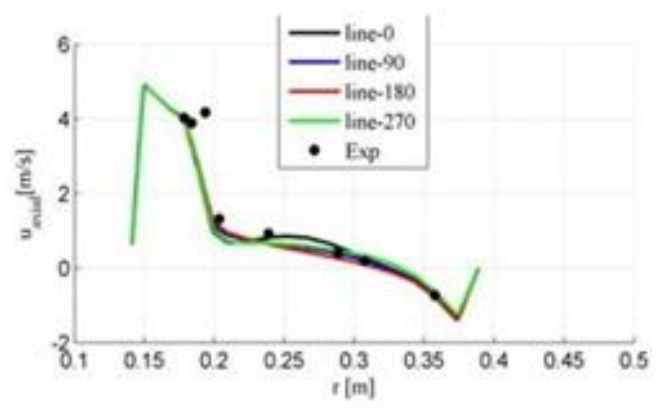

(a)

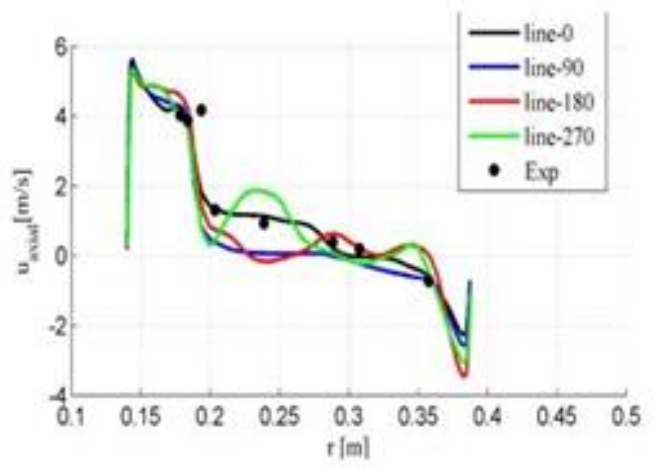

(c)

Trapped

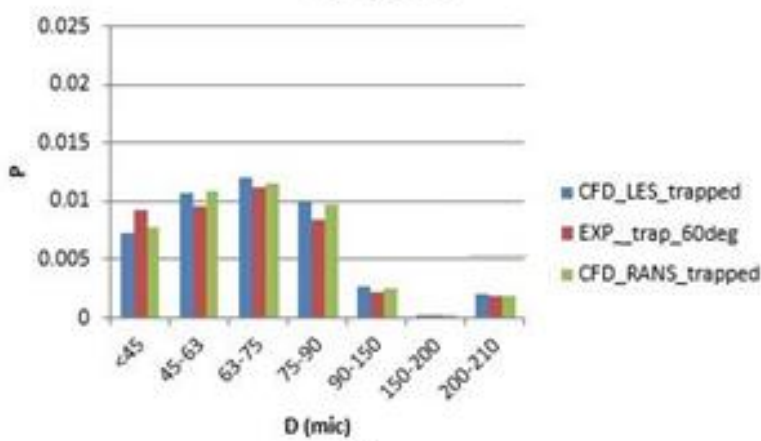

(e)

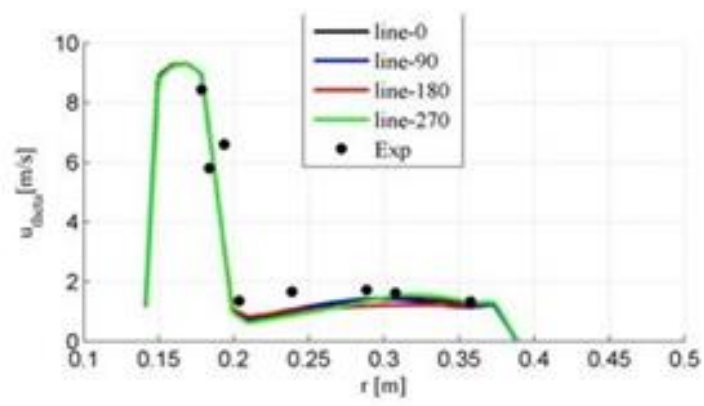

(b)

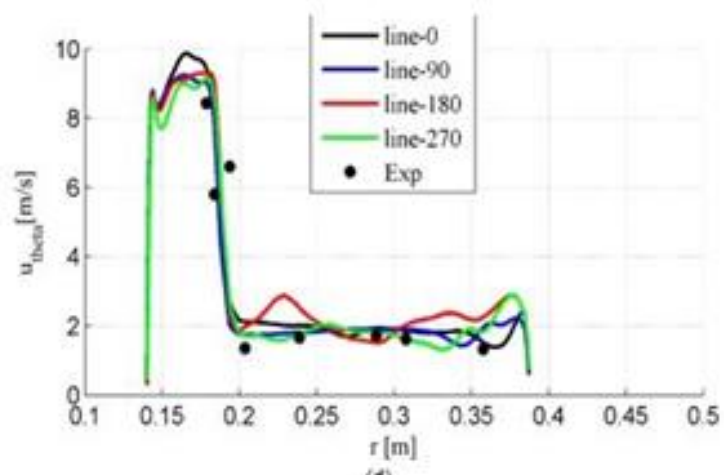

(d)

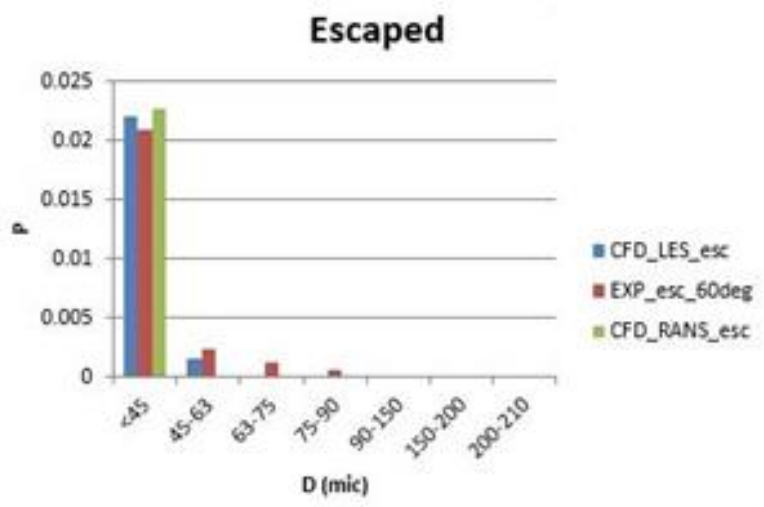

(f)

Figure 2.5 RANS vs LES results: axial gas velocity profiles - a) RANS c) LES respectively; tangential velocity profiles - b) RANS d) LES respectively; e) trapped conditional probability, f) escaped conditional probability

In spite of significant improvements in the results, still the larger size ranges in escaped particles are not being captured well by the simulations. This results in a pseudo high efficiency of the cyclone from the simulations. After studying and ruling out all the major possible factors that 
might affect the above particle behavior, this anomaly is now believed to be due to certain gassolid dynamics that the model is not able to account for.

\section{c) Not Including Rope / Cluster effects in the CFD Model}

According to [8], eddy flow can result in particles accumulating on the wall and secondary circulation deteriorates cyclone performance. This finding from the above work definitely gives an insight about the nature of the possible phenomenon that the models are not able to handle resulting in inaccuracies in efficiency prediction. This kind of deposition of particles occurs on the lower walls of pipes after gas-solid flows pass through geometries such as bends and ducts. High particle concentration regions are formed which resemble ropes. These result in slowing down of particles in high concentration regions and eventually lead to formation of deposits.

Figure 2.6 below shows the high particle concentration regions at the exit plane of the vane set from the earlier Axial Cyclone Separator simulations which we believe are precursors to roping. The bluff body has been hidden for clarity. These high particle concentration regions or ropes eventually lead to deposition of particles and hence we do not see particles of larger sizes in the escaped particles distribution. This is believed to be the phenomenon which causes anomalies in CFD gas-solid flow predictions.

Putting all these puzzles together, it can be concluded that a possible reason for the inaccurate numerical predictions of cyclone performance was hypothesized to be the CFD models not taking into account the roping phenomenon effect. 


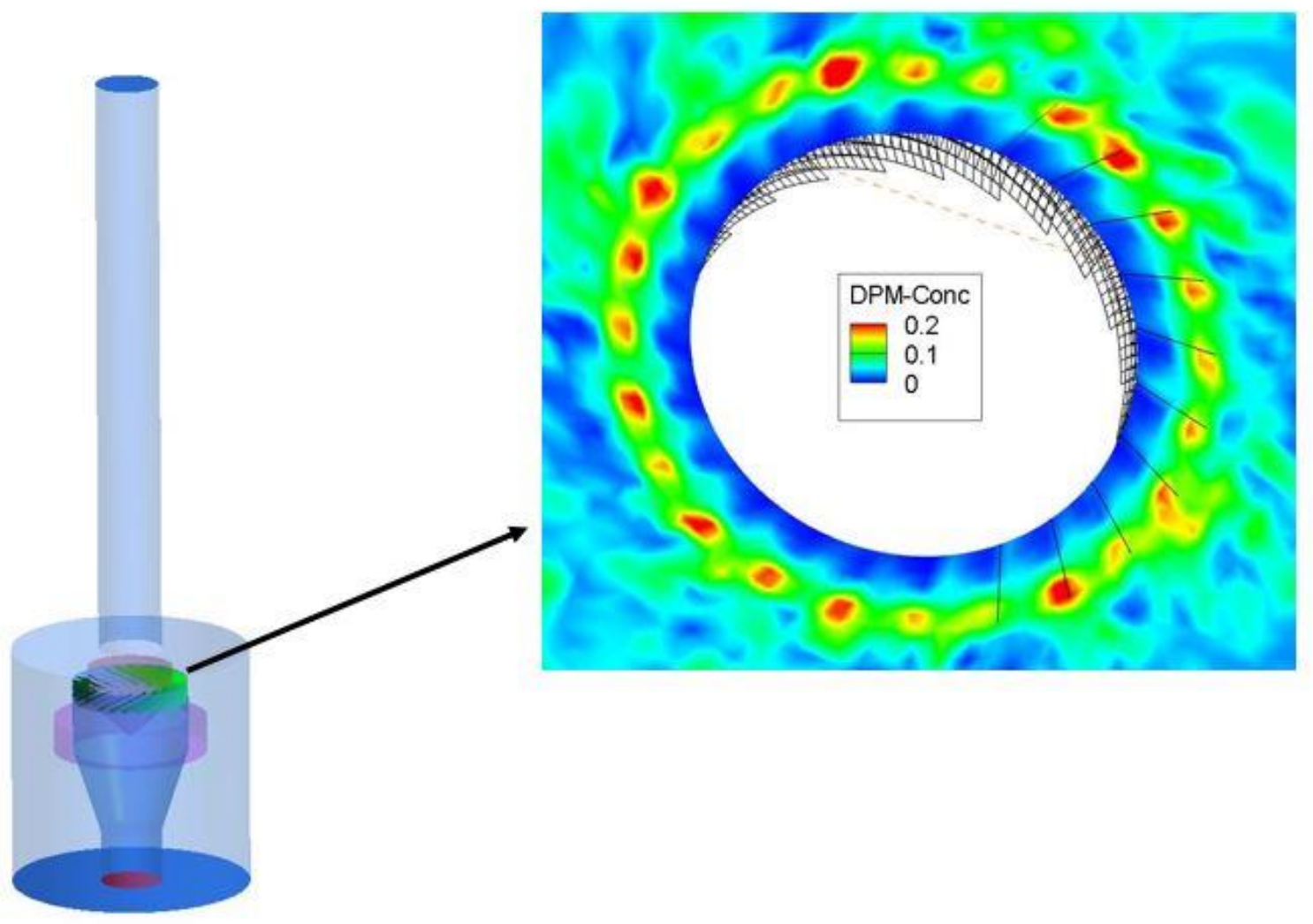

Figure 2.6 High particle concentration regions or ropes at the exit plane of the vane set in an Axial Cyclone Separator 


\section{Chapter 3 Literature Review}

In pneumatic conveying systems, the presence of ropes leads to problems with flow control and measurement within the power plant, and adversely affects the ability to reduce $\mathrm{NO}_{\mathrm{x}}$ emissions and leads to increased levels of unburnt carbon in the fly ash [9]. So there is a great need to accurately study the roping behavior in order tackle this problem. Hence modeling plays a vital role in understanding the physics of roping phenomenon.

\subsection{Turbulence Modelling}

Effectively capturing a turbulent flow field is a difficult task as the flow properties change a lot in a random and chaotic way in space and time. The flow has eddies with a wide range of space and time scales [10]. Great efforts are being made to develop methods that capture and define physics associated with turbulence. The methods may be broadly classified into three main categories Direct Numerical Simulation (DNS), turbulence models for Reynolds Averaged Navier - Stokes (RANS) equations and Large Eddy Simulation (LES).

In the DNS method, the Navier-Stokes equations are solved for all space and time scales [11]. In this regard, spatial grids should be extremely fine so that the Kolmogorov length scales at which energy dissipation takes place can be resolved. The time steps are very small to resolve periods of fast fluctuations. DNS is highly computationally expensive and is not practical for industrial applications due to limited resources. However, DNS is a highly valuable tool for fundamental research and model validation. The RANS method focuses on characterization based on the mean flow and the fluctuations [12]. This is known as Reynolds decomposition which when performed on the Navier-Stokes equations gives rise to additional terms known as Reynolds Stresses. These additional terms are modeled with classical turbulent closure models (Mixing length, k- $\epsilon, \mathrm{k}-\omega$ etc.). RANS-based CFD provides a reasonable compromise between accuracy and expense. Hence it is 
widely used in industrial applications. Finally in the LES method, the Navier-Stokes equations are solved after a filtering process based on a filter width [13]. The filtering process separates the smaller eddies from the ones larger than the filter width.The larger ones are directly resolved. The filtered scales are modeled using a sub grid scale model. LES is less computationally expensive than DNS but still requires high computational resources.

\section{RNG k- $\varepsilon$ (RANS) Turbulence Model}

By taking the time average of Navier-Stokes equations together with Reynolds decomposition, one can obtain Reynolds Averaged Navier-Stokes (RANS) equations.

The time-averaged momentum equations are:

$$
\frac{\partial U}{\partial t}+\operatorname{div}(U \mathrm{U})=-\frac{1}{\rho} \frac{\partial P}{\partial x}+v \operatorname{div}(\operatorname{grad}(U))+\frac{1}{\rho}\left[\frac{\partial\left(-\rho \overline{u^{\prime 2}}\right)}{\partial x}+\frac{\partial\left(-\rho \overline{u^{\prime} v^{\prime}}\right)}{\partial y}+\frac{\partial\left(-\rho \overline{u^{\prime} w^{\prime}}\right)}{\partial z}\right]
$$

(Time-averaged $\mathrm{X}$ - momentum equation)

$$
\frac{\partial V}{\partial t}+\operatorname{div}(V U)=-\frac{1}{\rho} \frac{\partial P}{\partial y}+v \operatorname{div}(\operatorname{grad}(V))+\frac{1}{\rho}\left[\frac{\partial\left(-\rho \overline{u^{\prime} v^{\prime}}\right)}{\partial x}+\frac{\partial\left(-\rho \overline{v^{\prime 2}}\right)}{\partial y}+\frac{\partial\left(-\rho \overline{v^{\prime} w^{\prime}}\right)}{\partial z}\right]
$$

(Time-averaged $\mathrm{Y}$ - momentum equation)

$$
\frac{\partial W}{\partial t}+\operatorname{div}(W \mathrm{U})=-\frac{1}{\rho} \frac{\partial P}{\partial z}+v \operatorname{div}(\operatorname{grad}(W))+\frac{1}{\rho}\left[\frac{\partial\left(-\rho \overline{u^{\prime} w^{\prime}}\right)}{\partial x}+\frac{\partial\left(-\rho \overline{v^{\prime} w^{\prime}}\right)}{\partial y}+\frac{\partial\left(-\rho \overline{v^{\prime} w^{\prime}}\right)}{\partial z}\right]
$$

(Time-averaged $\mathrm{Z}$ - momentum equation)

Though not shown here, the mass conservation equation is required and solved too. To solve RANS equations, the Reynolds stress terms needs to be modeled for the closure of the system. Several models and schemes have been proposed for addressing the closure problem such as linear eddy viscosity model. The RANS models are classified based on the number of additional transport equations that need to be solved along with the flow equations. The RNG $k-\epsilon$ model has been 
used in this study. The RNG $k-\epsilon$ model is a linear eddy viscosity model, and it is derived from renormalization group theory. Similar to other two equation $k-\epsilon$ models, turbulent kinetic energy $(k)$ and turbulent dissipation $(\epsilon)$ are solved from the following two extra transport equations [14]. Simulations performed with this model better matched with experimental data [15].

$$
\begin{gathered}
\frac{\partial(\rho k)}{\partial t}+\frac{\partial\left(\rho k u_{i}\right)}{\partial x_{i}}=\frac{\partial}{\partial x_{j}}\left(\alpha_{k} \mu_{e f f} \frac{\partial k}{\partial x_{j}}\right)+G_{k}+G_{b}-\rho \epsilon-Y_{M}+S_{k} \\
\frac{\partial(\rho \epsilon)}{\partial t}+\frac{\partial\left(\rho \epsilon u_{i}\right)}{\partial x_{i}}=\frac{\partial}{\partial x_{j}}\left(\alpha_{\epsilon} \mu_{e f f} \frac{\partial \epsilon}{\partial x_{j}}\right)+C_{1 \epsilon} \frac{\epsilon}{k}\left(G_{k}+C_{3 \epsilon} G_{b}\right)-C_{2 \epsilon} \rho \frac{\epsilon^{2}}{k}-R_{\epsilon}+S_{\epsilon}
\end{gathered}
$$

In these equations, $G_{k}$ is the turbulence kinetic energy generation due to the mean velocity gradients, $G_{b}$ is the turbulence kinetic energy generation due to buoyancy and $Y_{M}$ is the contribution of the fluctuating dilatation in compressible turbulence to the overall dissipation rate. The $\alpha_{k}$ and $\alpha_{\epsilon}$ are the inverse effective Prandtl numbers for $k$ and $\epsilon$ respectively.The $S_{k}$ and $S_{\epsilon}$ are user-defined source terms. The default values of $C_{1 \epsilon}$ and $C_{2 \epsilon}$ are 1.42 and 1.68 respectively. The turbulence (eddy) viscosity is defined as

$$
\mu_{t}=\rho C_{\mu} \frac{k^{2}}{\epsilon}
$$

where $C_{\mu}=0.0845$.

\section{Smagorinsky-Lilly (LES) model}

LES is based on a filtering operation to separate large scales from the small scales. The governing equations are thus obtained by filtering the time dependent Navier Stokes equations. A filtered variable is defined as:

$$
\bar{\phi}(\mathrm{x})=\int_{D} \phi\left(x^{\prime}\right) G\left(x, x^{\prime}\right) d x^{\prime}
$$


Where $\mathrm{D}$ is the domain and $\mathrm{G}$ is the filter function that determines the scale of the resolved eddies. The filtering operation results in the filtered equations of motion. For an incompressible flow, they are:

$$
\begin{gathered}
\frac{\partial \bar{u}_{i}}{\partial \bar{x}_{i}}=0 \\
\frac{\partial \bar{u}_{i}}{\partial t}+\frac{\partial}{\partial x_{j}}\left(\bar{u}_{i} \bar{u}_{j}\right)=-\frac{1}{\rho} \frac{\partial \bar{p}}{\partial x_{i}}-\frac{\partial \tau_{i j}}{\partial x_{j}}+v \frac{\partial^{2} \bar{u}_{i}}{\partial x_{j} \partial x_{j}}
\end{gathered}
$$

The effect of small scales appears through a subgrid scale (SGS) stress term, $\tau_{i j}$. The subgrid scale stresses which arise due to the filtering operation are unknown and hence require modeling. The subgrid scale models calculate the subgrid scale turbulent stresses from:

$$
\tau_{i j}-\frac{1}{3} \tau_{k k} \delta_{i j}=-2 \mu_{t} \bar{S}_{i j}
$$

This model was first proposed by Smagorinsky [16]. The eddy viscosity is given by:

$$
\mu_{t}=\rho L^{2}{ }_{s}|\bar{S}|
$$

Here $L_{S}$ is the mixing length for subgrid scales and

$$
|\bar{S}|=\sqrt{2 \bar{S}_{i j} \bar{S}_{i j}}
$$

In FLUENT, $L_{S}$ is calculated as follows:

$$
L_{s}=\min \left(K d, C_{s} V^{\frac{1}{3}}\right)
$$


Here $K$ is the von Kármán constant, $d$ is the distance to the closest wall, $C_{S}$ is the Smagorinsky constant and $V$ is the volume of a computational cell.

Lilly calculated a value of 0.17 for $C_{S}$ for homogenous isotropic turbulence in the inertial subrange. $C_{S}$ is not a universal constant. This is a drawback with this model. The value of $C_{S}=0.1$ has been used in this study.

\subsection{Numerical Modelling of Gas-Solid Flows}

\section{a) Eulerian -Lagrangian Approach}

In this study, an Eulerian-Lagrangian approach is used for the gas-solid flow simulation. In this approach, the gas phase is treated as a continuum phase, and the solid particles are considered as a discrete phase. The gas phase computations are done with the Navier-Stokes equations used as the governing equations. The motion of the solid particle phase is represented by the Lagrangian model approach where the discrete particle phase is tracked by solving the equations of motion in a Lagrangian reference framework.

The general equation for gas -phase is given by:

$$
\frac{\partial}{\partial t}(\rho \vec{v})+\nabla \cdot(\rho \vec{v} \vec{v})=-\nabla p+\nabla \cdot(\overline{\bar{\tau}})+\rho \vec{g}+\vec{F}
$$

Here $F$ represents external body forces such as due to interaction with the dispersed phase.

The motion of solid particles is governed Newton's second law. By considering all the forces acting on the particle, the equation of motion can be written as

$$
\rho_{p} \frac{d \vec{U}_{p}}{d t}=\overrightarrow{\mathrm{F}}_{d}+\overrightarrow{\mathrm{F}}_{b}+\overrightarrow{\mathrm{F}}_{V M}+\overrightarrow{\mathrm{F}}_{f p}+\overrightarrow{\mathrm{F}}_{p p}
$$


where $\overrightarrow{\mathrm{F}}_{d}, \overrightarrow{\mathrm{F}}_{b}, \overrightarrow{\mathrm{F}}_{V M}, \overrightarrow{\mathrm{F}}_{f p}$, and $\overrightarrow{\mathrm{F}}_{p p}$ represent the drag force, buoyancy force, virtual mass force, fluid-particle interaction, and particle-particle interaction respectively. In the present study, the particle-particle interactions are not taken into account. Only the drag force is taken into account and all the other forces are neglected since the ratio of density of particles to that of gas is of the order $10^{3}$. The drag force is defined as

$$
\vec{F}_{d}=F_{D}\left(\vec{u}_{g}-\vec{u}_{p}\right)
$$

with the coefficient

$$
F_{D}=\frac{18 \mu C_{D} R e}{d_{p}^{2} 24}
$$

and relative Reynolds number

$$
R_{e}=\frac{\rho d_{p}\left|\vec{u}_{g}-\vec{u}_{p}\right|}{\mu}
$$

Here $\mathrm{C}_{\mathrm{D}}$ is drag coefficient based on the non-spherical drag law.

$$
C_{D}=\frac{24}{R e}\left(1+b_{1} R e^{b_{2}}\right)+\frac{b_{3} R e}{b_{4}+R e}
$$

where

$$
\begin{aligned}
& b_{1}=\exp \left(2.3288-6.4581 \varphi+2.4486 \varphi^{2}\right) \\
& b_{2}=0.0964+0.5565 \varphi \\
& b_{3}=\exp \left(4.905-13.8944 \varphi+18.4222 \varphi^{2}-10.2599 \varphi^{3}\right) \\
& b_{4}=\exp \left(1.4681+12.2584 \varphi-20.7322 \varphi^{2}+15.8855 \varphi^{3}\right)
\end{aligned}
$$

and $\varphi$ is known as the shape factor. Here Re is calculated with diameter of a sphere having the same volume. 
The Discrete Phase Model (DPM) of Ansys FLUENT is used for calculating the trajectory of each individual particle. Ansys FLUENT predicts the trajectory of a discrete phase particle by integrating the particle equation of motion written in a Lagrangian reference frame [17]. It does a force balance by equating particle inertia with all the forces acting on the particle. Transient simulations have been performed with Unsteady Particle Tracking.

\section{b) Eulerian -Eulerian Approach}

In this approach, both the phases are treated as a continuum. Each phase is represented by volume fractions and the conservation equations are solved for each phase. The volume $V_{q}$ of a phase $q$ with a volume fraction of $\alpha_{q}$ is given by the following equation:

$$
V_{q}=\int_{V} \alpha_{q} d V
$$

where

$$
\sum_{p=1}^{n} \alpha_{q}=1
$$

The effective density of a phase $q$ is given by:

$$
\hat{\rho}_{q}=\alpha_{q} \rho_{q}
$$

where $\rho_{q}$ is the actual density of the phase.

Volume fraction of each phase is calculated from the continuity equation. According to [17], the continuity equation for a phase $q$ is given by:

$$
\left(\frac{\partial}{\partial t}\left(\alpha_{q} \rho_{q}\right)+\nabla \cdot\left(\alpha_{q} \rho_{q} \vec{v}_{q}\right)\right)=0
$$


Here $\vec{v}_{q}$ is the velocity of phase $q$. The solution of the above continuity equation for each secondary phase and the summation of volume fractions being equal to 1 results in calculation of volume fraction of primary phase.

The conservation of momentum equation for a phase $q$ is given by:

$$
\begin{aligned}
\frac{\partial}{\partial t}\left(\alpha_{q} \rho_{q} \vec{v}_{q}\right)+ & \nabla \cdot\left(\alpha_{q} \rho_{q} \vec{v}_{q} \vec{v}_{q}\right) \\
& =-\alpha_{q} \nabla p+\nabla \cdot \overline{\overline{\tau_{q}}}+\alpha_{q} \rho_{q} \vec{g}+\sum_{p=1}^{n}\left(\vec{R}_{p q}\right) \\
& +\left(\vec{F}_{q}+\vec{F}_{l i f t, q}+\vec{F}_{w l, q}+\vec{F}_{v m, q}+\vec{F}_{t d, q}\right)
\end{aligned}
$$

where $\vec{g}$ is acceleration due to gravity, $\overline{\tau_{q}}$ is the $q^{\text {th }}$ phase stress-strain tensor, $\vec{F}_{q}$ is an external body force, $\vec{F}_{l i f t, q}$ is lift force, $\vec{F}_{w l, q}$ is wall lubrication force, $\vec{F}_{v m, q}$ is virtual mass force and $\vec{F}_{t d, q}$ is turbulent dispersion force. Here $\vec{R}_{p q}$ is an interaction force between phases and is given by:

$$
\sum_{p=1}^{n}\left(\vec{R}_{p q}\right)=\sum_{p=1}^{n}\left(K_{p q}\left(\vec{v}_{p}-\vec{v}_{q}\right)\right)
$$

where assuming secondary phase forms droplets or bubbles, $K_{p q}$, the fluid-fluid exchange coefficient defined as:

$$
K_{p q}=\frac{\rho_{p} f}{6 \tau_{p}} d_{p} A_{i}
$$

where $d_{p}$ is diameter of droplets or bubbles of phase $p, f$ is drag function, $\tau_{p}$ is defined as particulate relaxation time and $A_{i}$ is the interfacial area. 


$$
\begin{gathered}
\tau_{p}=\frac{\rho_{p} d_{p}^{2}}{18 \mu_{q}} \\
A_{i}=\frac{6 \alpha_{p}}{d_{p}}
\end{gathered}
$$

This Eulerian-Eulerian model has been used in both FLUENT and MFIX with similar basic equations.

\subsection{Modeling Issues in Classifiers}

The inconsistencies between experiments and simulations (Chapter 2) in prediction of functioning and efficiency of cyclone classifiers is believed to be due to formation of high concentration regions known as ropes. In order understand the physics behind roping, it is of utmost importance to first accurately capture this phenomenon and also study the factors affecting it. It is also necessary to study the clustering behavior of particles as roping is similar to particle clustering in the sense that both result in formation of high particle concentration regions.

\section{Roping Phenomenon}

Roping in gas-solids flows is a phenomenon in which high particle concentration regions are formed as a result of inertial effects of change in flow direction. Figure 3.1 below shows roping in a horizontal pipe after a vertical to horizontal pipe bend.

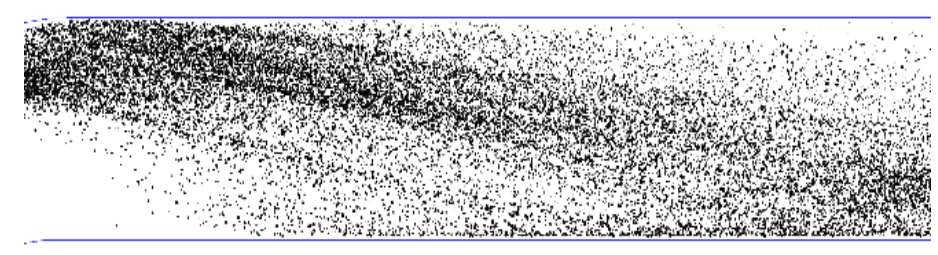

Figure 3.1 Visible rope in a horizontal pipe after a $90^{\circ}$ bend exit

Several researchers have studied rope formation under a wide range of conditions. The ropes carry the bulk of the transported material concentrated within a small cross-section of the pipe with 
lower particle velocities and relatively high solids concentrations. Yilmaz and Levy [18] studied formation of ropes by fine powdered coal through a 90 degree horizontal to vertical (upward) elbow for air-to-solids loading ratios of 1 to 3 . In this study, they used a fiber optic probe to measure particle velocities and concentrations. Their results showed that ropes would form and initially move towards the outer wall of the pipe bend. These are later brought back towards the mid-section of the pipe by secondary flows, where the flow turbulence would then disperse them. Also they found that the rope dispersion could be accelerated by the introduction of an orifice plate into the flow. They also concluded that several flow structures prevail in both the axial and radial directions in the pipe downstream of the elbow.

Akilli et al.[19] performed both experiments and simulations on gas-solid flow behavior in a $90^{\circ}$ vertical-horizontal pipe bend for air-solids loadings of 1 and 3. Particle velocity profiles and concentrations from experiments were obtained by means of a fiber optic probe. They used the CFX CFD package for performing the simulations. They concluded that the formation and disintegration of these ropes is a complex phenomenon that is influenced by several factors such as centrifugal forces, secondary flows, gas velocity, pipe geometry, bend orientation and physical properties of the transported material.

Yilmaz and Levy [9] conducted experiments and simulations to examine the effects of different elbow radius of curvature / pipe diameter (R/D) ratios on rope formation and dispersion. They found in their experiments that there was a strong effect of the R/D ratio on the rate of rope formation and dispersal. The higher the R/D ratio, the slower the rate of rope dispersal was observed. They reported that the CFD results over predicted the peak particle concentrations within the rope at the elbow exit and concluded that this was most likely due to the absence of particle-particle interactions within the CFD model. The simulation results also showed that 
secondary flows dispersed the rope by carrying particles around the circumference of the pipe whereas turbulence dispersed the rope by localized mixing of particles.

Huber and Sommerfeld [20] performed experiments to characterize the cross-sectional particle concentrations. They used glass beads as the conveyed material and also studied the effect of wall roughness by using glass and stainless steel pipes. Measurements were made using laser-light sheet method and also Phase-Doppler Anemometry. The roughness of the pipe was found to have a great influence on particle motion after wall-collision. For the smooth walled pipes, the particle concentration was observed to be more towards the lower wall. However for the rough walled pipes, the concentration is more in the core region of the pipe due to a re-suspension effect of wall roughness. Also the concentrations were found to be fairly asymmetric in the smooth walled case. However only slight asymmetry was observed in the rough walled case.

Kliafas and Holt [21] performed LDV measurements of mean-stream wise and radial velocities in an air-solid two phase flow in a square cross-sectioned $90^{\circ}$ vertical to horizontal pipe bend. Glass spheres of two different sizes were used in these experiments. The velocity values were found to be maximum at the inner wall due to a favorable stream wise pressure gradient, and there was a deceleration towards the outer wall as a result of unfavorable pressure gradient. It was observed that the velocity profiles of gas and solids cross over at outer walls. It was also found that the turbulence for solids was very high compared to the gas except near the outer wall region as the curvature increased gas turbulence.

Ibrahim et al. [15] performed simulations comparing the predictions using both the standard k- $\varepsilon$ model and RNG k- $\varepsilon$ model. The RNG k- $\varepsilon$ model was found to have a better agreement with the experimental data compared to the standard $k-\varepsilon$ model. Also the influence of other factors like particle diameter, mass-loading, curvature-ratio and inlet velocity were also studied. It was found 
that the particle concentration increased towards the outer wall with increase in particle diameter. For higher values of curvature-ratio and particle diameter, a sudden increase in normalized axial particle velocity was observed near the outer wall.

Kuan and Yang [22] investigated the behavior of dilute turbulent particulate flows in a curved $90^{\circ}$ horizontal to vertical bend using 2D Laser Doppler Anemometry. Glass spheres were released into a square cross-sectioned duct. Significant gas-solid separation was observed near the outer wall of the duct, and the region was characterized by considerable positive slip velocity between the two phases.

Njobuenwu et al. [23] modeled a particle-laden turbulent flow in a square cross-sectioned duct with a $90^{\circ}$ bend using a three-dimensional Eulerian-Lagrangian approach. It has been observed that strong pressure gradients influence stream wise velocity near the inner walls of the bend. Upstream of the bend, the flow is accelerated near inner radius wall due to favorable pressure gradient and is decelerated near outer radius wall due to adverse pressure gradient. Downstream of bend, maximum velocity is shifted towards outer wall due to centrifugal effects. The particulate flow was found to be influenced by the instantaneous gas-phase flow, the particle-size distribution, and particle wall collisions.

Kruggel-Emden and Oschmann [24] studied the effects of various particle shapes on rope dispersion in a horizontal-to-vertical (upwards flow) elbow using coupled DEM-CFD simulations. Their results indicated that the rate of rope dissipation was strongly affected by particle shape, where icosahedrons provided the most rapid rope dispersion, and plates the lowest. They went on to observe the general trend that strong particle-fluid interaction, particle-particle collisions and large bend exit velocities result in accelerated rope dispersion. 
Guda et al. [25] presented a model validation study as well as a study on rope formation in a vertical to horizontal elbow using both ground flaxseed and HDPE (high density polyethylene) pellets for a variety of particle size distributions, and found that the simulation results obtained from using a RANS model in FLUENT failed to accurately predict the onset of roping with HDPE pellets at solids loadings less than 1.5. In their study, the simulation results suggested the presence of rope formation for solids-to-air loadings which did not result in roping under experimental conditions.

The factors affecting the rope formation such as pipe geometry, inlet particle velocities, solids-gas loadings have been studied and presented by many researchers over the years. However the fundamental question of how the rope is formed by flow physics is not defined. The main objective of the study is to investigate the actual role of flow in formation of rope which is missing in previous studies. In many studies, the presence or formation of rope is taken for granted by relating it to geometry alone. Also, it is necessary to postulate a fundamental definition of rope and to investigate why and how the particles cluster to form ropes. In this study, effects of other factors such as variation of method of loading, inlet turbulent length scale, inlet turbulent intensity, wall coefficient of restitution and particle mean diameter based slip velocity calculation are presented. This study mainly provides good flow visualization of roping phenomenon for different solid-gas loadings through both experiments and simulations. This study will provide the base for understanding flow behavior in classifiers and to predict classifier efficiency accurately.

\section{Clustering Phenomenon}

Several experiments and simulations performed by researchers resulted in the study of formation of particle clusters in fluidized beds. 
McMillan et al. [26] performed experiments with high speed imaging in fluidized beds and risers and observed clustering phenomenon clearly. They found that small particles frequently form clusters. They also found that clusters in fluidized bed region are larger than those in freeboard region. At high superficial gas velocities, large clusters were observed near walls and at low velocities smaller clusters were observed. It was also observed that particle clusters are held together by weak forces and can break up into smaller clusters easily. Experimentally, they also proposed that hydrodynamics are due to drag forces, i.e. as fluid interacts with particles, low pressure eddies are created which attract other particles.

Cocco et al. [27] in their experiments aided by high speed imaging showed that clusters depend not only on the fine particles level but also on the material itself; in their comparison between polyethylene material and FCC catalyst. They found that cluster formation is dominant in the bed region and these get transported to freeboard region, even though cluster formation in freeboard region occurs too. Also they found that particles which were smooth were prone to clustering.

Gokaltun et al. [28] used shadow sizing technique with high speed imaging in their experiments and observed cluster formation by observing the deviation in particle volume fraction from the mean value. The observed values of instantaneous volume fraction of particles and the particle diameters were at least twice the standard deviation.

An et al. [29] observed formation of clusters in the form of horizontal strands in two- dimensional liquid-solid fluidized beds by using charge coupled devices (CCD) imaging and measuring technique. They observed that during fluidization, the motion of solid particles upward in the fluidized bed is in the form of groups or clusters. They observed that the clusters are in the form of horizontal strands. 
Gomez and Milioli [30] observed that the traditional two fluid model adequately captured the mean macroscopic features of the gas-solid flows in a circulating fluid bed riser. This includes particle clustering along with annular plug flow patterns. They also suggested that theoretically it is difficult to refine the mesh beyond a certain limit and still maintain the validity of continuum formulation. They also suggested usage of adaptive mesh procedures instead.

Vaishali and Roy [31] performed numerical simulations with Geldart Group B Particles. They performed a 2-D axisymmetric simulation for the current case which resulted in unrealistic results which were not reliable. They observed that 3D simulations should be performed as they are very accurate but at the same time expensive in terms of resources.

Benyahia et al. [32] performed 2-D transient simulations using CFX package for a riser. They observed that core annual flow is significantly affected by downward flow of solid near the wall region. They suggested that a long time averaging is necessary to compare computational results with the experimental data in the riser due to different frequencies of large scale fluctuations.

Tsuji et al. [33] compared a discrete phase model and a two fluid model in their numerical study of cluster formation. They observed that population of clusters is larger in discrete phase model compared to the two-fluid model. They also observed that as duct sizes increases, clusters not only form at wall but also in the central region of the duct using discrete phase model. However, they found that clusters disappear as duct size increases using two-fluid model.

Zhou et al. [34] studied the turbulent gas-particle flow in a bubbling fluidized bed using LES-DPM method. The gas-phase model is based on 2D Navier-Stokes equations for two-phase flow with turbulence calculated by LES. The particles were treated by a DPM model. They observed a circulation pattern based on how particles rise in the center of the bed and fall down near walls. 
The anisotropic particle velocity distributions indicated that particles did not have a local equilibrium.

Li et al. [35] observed that it is impossible to match both axial solids hold up and radial solids concentration for a $2 \mathrm{D}$ simulation with $3 \mathrm{D}$ data. They observed that $2 \mathrm{D}$ simulations can only be used for quantitative studies while 3D numerical simulations are needed to accurately capture quantitative flow behavior.

Capacelatro et al. [36] performed a large eddy simulation based on Eulerian-Lagrangian technique and their numerical results matched with experiments very well. They observed inconsistencies in $2 \mathrm{D}$ and $3 \mathrm{D}$ simulations of risers i.e. $2 \mathrm{D}$ simulations of risers lead to unphysical accumulation of particles due to their restriction of motion in a plane.

Roping is similar to particle clustering in the sense that both have high particle concentration regions. In order to understand roping, it is necessary to first understand clustering. Past studies done by researchers have dealt with how cluster properties are affected by several factors. The role of flow dynamics in cluster formation and effects of vorticity on clusters is not clearly identified. Whether the clusters cause vorticity or vorticity results in cluster formation is not clear and a deeper understanding is missing. Hence the objective of this study is to elucidate the cause and effect relations behind vorticity and particle clustering. 


\section{Chapter 4 Roping and Clustering Phenomenon}

\subsection{Introduction}

Transportation of materials is a very important aspect in industry. Whether it is transportation of finished products or raw materials, it plays a vital role in the economics as well as healthy functioning of an industry. Materials are transported manually or by mechanical means depending upon the nature of the material being transported. Not all materials can be transported by the same means. For instance, combustible materials require extra care and precautions in transport compared to non-combustible ones. Fluids require a different transportation mechanism compared to solids. Rare and precious materials require careful transport compared to the easily available and cheap materials. As the properties and the nature of materials change, the transportation mechanism and the manner changes. There are several means of material transport in the industry. Pneumatic conveying, gravity chutes, air slides, belt conveyors, screw conveyors, bucket elevators, vibratory conveyors are some of the popular methods.

Pneumatic conveying is a popular means of material transport in the industry especially for powdered and pulverized materials. Chemical, pharmaceutical, food processing, and power generation industries are some examples where pneumatic conveying plays a vital role. Pneumatic conveying is basically the process of material transport through pipelines with gas as the carrier. Generally compressed air from an air compressor carries the material through pipes from one station to another. Materials generally transported by this method are dry and free flowing. Flexibility in operation, easy routing and spacing, safe working conditions and low maintenance cost are some of the advantages of this method. 
A fluidized bed is formed by the introduction of pressurized fluid through particulate medium being held in a holding vessel which in turn makes the mixture behave as a fluid. Fluidized beds have a wide range of applications in industry. Fluidized bed reactors are used for energy production such as chemical looping combustion and gasification processes. Fluidized beds are also used for drying, cooling and granulation of powders. They are also popularly used for catalytic cracking.

In both pneumatic conveying and the functioning of fluidized beds, the most common underlying mechanism is air acting as a carrier medium for the solid particles. The gas-solid behavior in these fluidized beds and pneumatic conveying method is similar. Regions of high particle concentration are observed due to gas-solid segregation and accumulation. These regions are known as ropes in pneumatic conveying, through pipe bends and ducts; and known as clusters in fluidized beds. There is accumulation of solids in these regions.

\subsection{Roping in a $90^{\circ}$ Pipe Bend}

In order to make the plant layouts simple, numerous bends and pipe sections are introduced in the piping layouts. The phenomena that occur when material is transported through these ducts and bends is very interesting. For example, coal-fired power plants operate on a continuous supply of pulverised coal transported from the mill through these ducts. The piping used in these transport systems commonly includes bends that have a significant effect on the gas-solid flow structure. Due to centrifugal forces within these pipe bends, the gas and solid particles segregate and the particles form a dense structure known as a rope. This region has relatively high particle concentration. Deposition of particles also occurs due to deceleration of particles. This phenomenon leads to difficulties for plant operators in maintaining optimal conditions for combustion in furnaces as a result of irregularities in the pulverised fuel supply. 
The main objective of this work is to effectively and accurately capture the roping phenomenon in gas-solid flows through a $90^{\circ}$ pipe bend through experiments and simulations with Ansys FLUENT.

\subsubsection{Experimental Study of Gas-Solid Flow in Pipe Bend}

Experiments generally provide a sound basis for numerical simulations and development of models. The main aim here is to capture roping phenomenon accurately in experiments and use this to validate the numerical study results later.

\section{A) System Description}

The experimental system has been designed with the main purpose of performing high speed imaging and to analyze particle behavior and characteristics from the high speed videos. Figure 4.1 shows the schematic diagram of the experimental system used in this study. This system was used to obtain high speed video data of roping under different loading conditions.
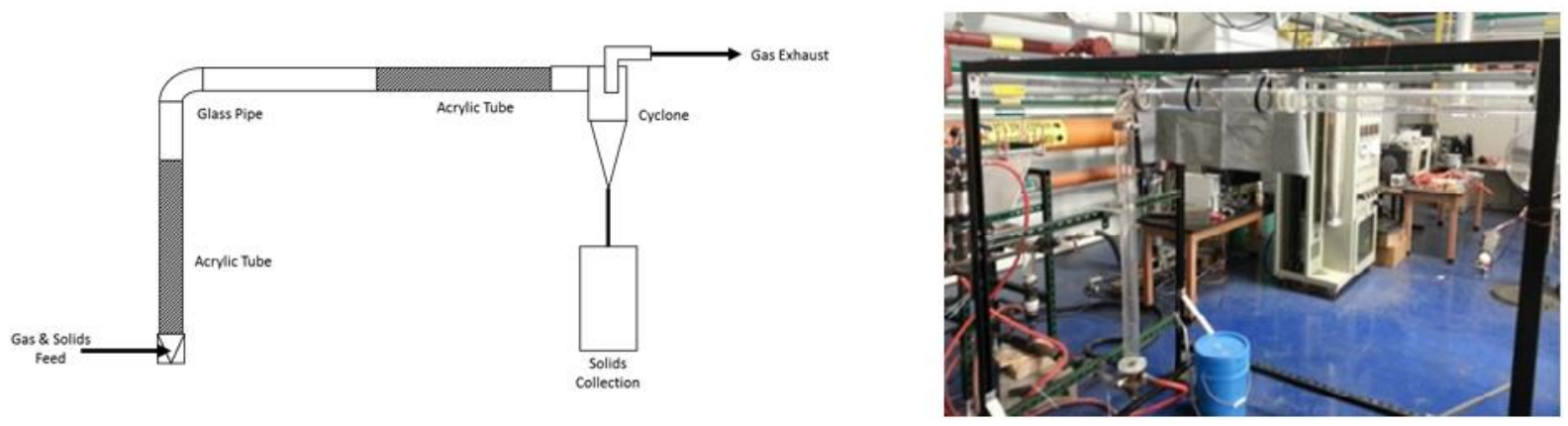

Figure 4.1 Experimental setup

The experimental unit consists of a vertical pipe section, a $90^{\circ}$ vertical-to-horizontal elbow, and a horizontal pipe section. The vertical pipe section is composed of a 2.5 inch inner diameter clear acrylic tube that is 36 inches in length; above the acrylic tube is a 3 inch inner diameter borosilicate glass pipe section that is 12 inches in length. The borosilicate glass elbow has a 3 inch inner diameter and a radius of curvature to pipe diameter ratio (R/D) of 1.8. The horizontal section 
consists of a 3 inch inner diameter by 36 inch long borosilicate glass pipe, followed by a 2.5 inch diameter by 36 inch long clear acrylic tube. The outside diameter of the acrylic tubes for both the vertical and horizontal sections were machined down on the ends to allow the tubes to fit inside the borosilicate glass pipes. These joints were sealed to prevent air leakage. The inside diameters of the ends of the acrylic tubes were also machined to allow smooth transition from the 2.5 inch diameter of the acrylic to the 3 inch diameter of the glass sections. A screw feeder was used to introduce solid particles into the bottom of the unit via a pneumatic transport line. A large capacity air compressor provided the required airflow for the experiments. This air was introduced into the experimental unit via a conical-shaped air distributor, where the flowrates were regulated via a series of variable-area style rotameters. A particle cyclone separator was attached to the end of the horizontal pipe section, where the solid particles were separated from the airflow exiting the system and collected for reuse.

While the formation of ropes in pulverized coal-carrying pipe systems is of primary interest to this study, the fine coal dust deposition on the pipe walls prevents visual observation of the roping phenomenon. Several materials such as spent FCC catalyst, bulgur and glass beads were tried in a small glass prototype. However due to their hard abrasive nature, they damaged the glass by discoloring it. Since the main idea of conducting experiments is to obtain clear high speed videos of roping phenomenon, these materials have been avoided.

\section{B) Preliminary Experiments}

Preliminary experiments were performed with HDPE (High Density Polyethylene) and flaxseed for different loading conditions [25]. Roping behavior was observed with HDPE for very high mass loadings of above $100 \%$ and for flaxseed at about $42 \%$. Figure 4.2 and Figure 4.3 show sample snapshots from the slow motion videos. The reason behind not observing roping in HDPE 
case until a very high mass loading was due nature of particles. A lot of electrostatic charging was associated with those particles. Therefore, the solids material used in this study was ground flaxseed.

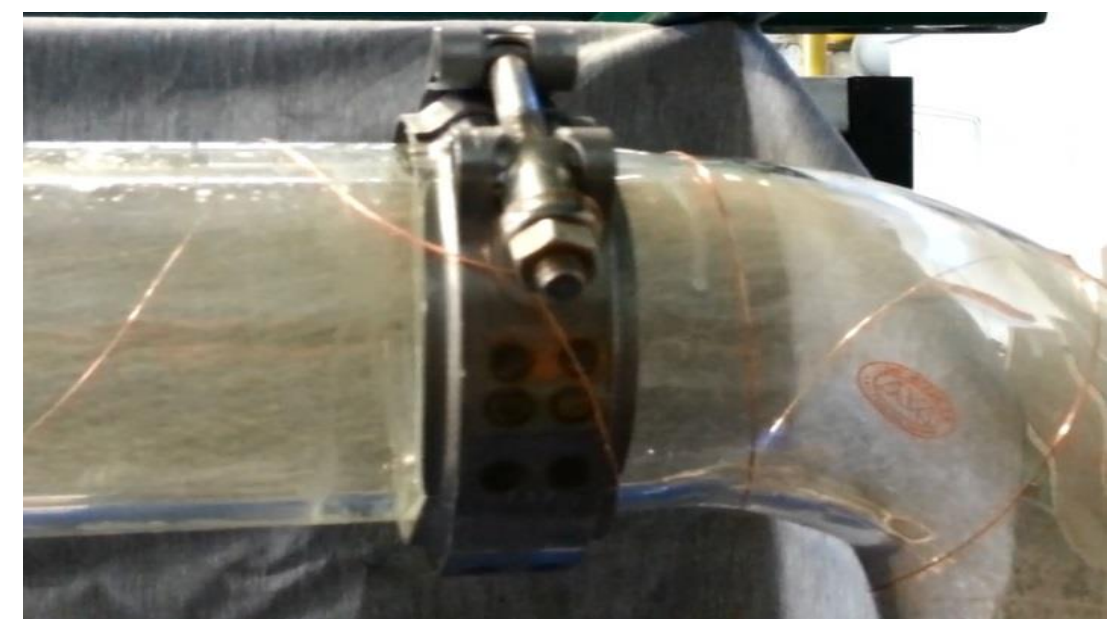

Figure 4.2 Snapshot of HDPE particles for a loading of $100 \%$

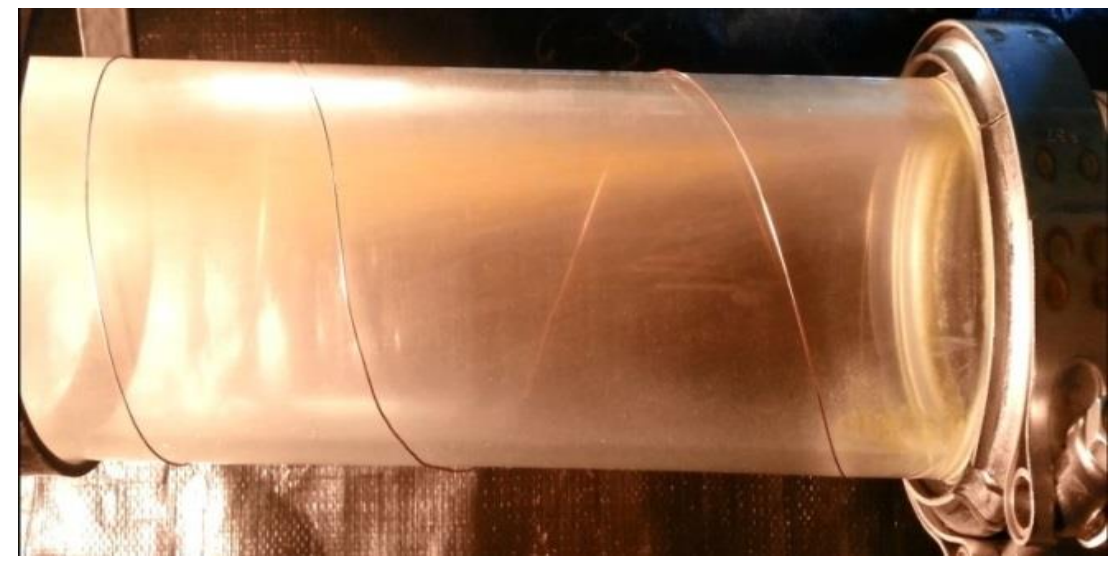

Figure 4.3 Snapshot of rope with flaxseed at loading of $42 \%$

\section{C) Flaxseed for Experiments}

The flaxseed was selected primarily because previous studies [25] showed it to be prone to roping at lower solids loadings, as well as being non-abrasive it would not scratch the glass. Also it is inexpensive and easily available. The particle size distribution for these particles is given in Figure 4.4, and other material properties can be found in Table 4.1. For this study, solids-to-air mass 
loadings of 35,42 and $51 \%$ were obtained by maintaining a constant solids mass feed rate and varying the flow rate of air. Table 4.2 provides the exact flow rates used.

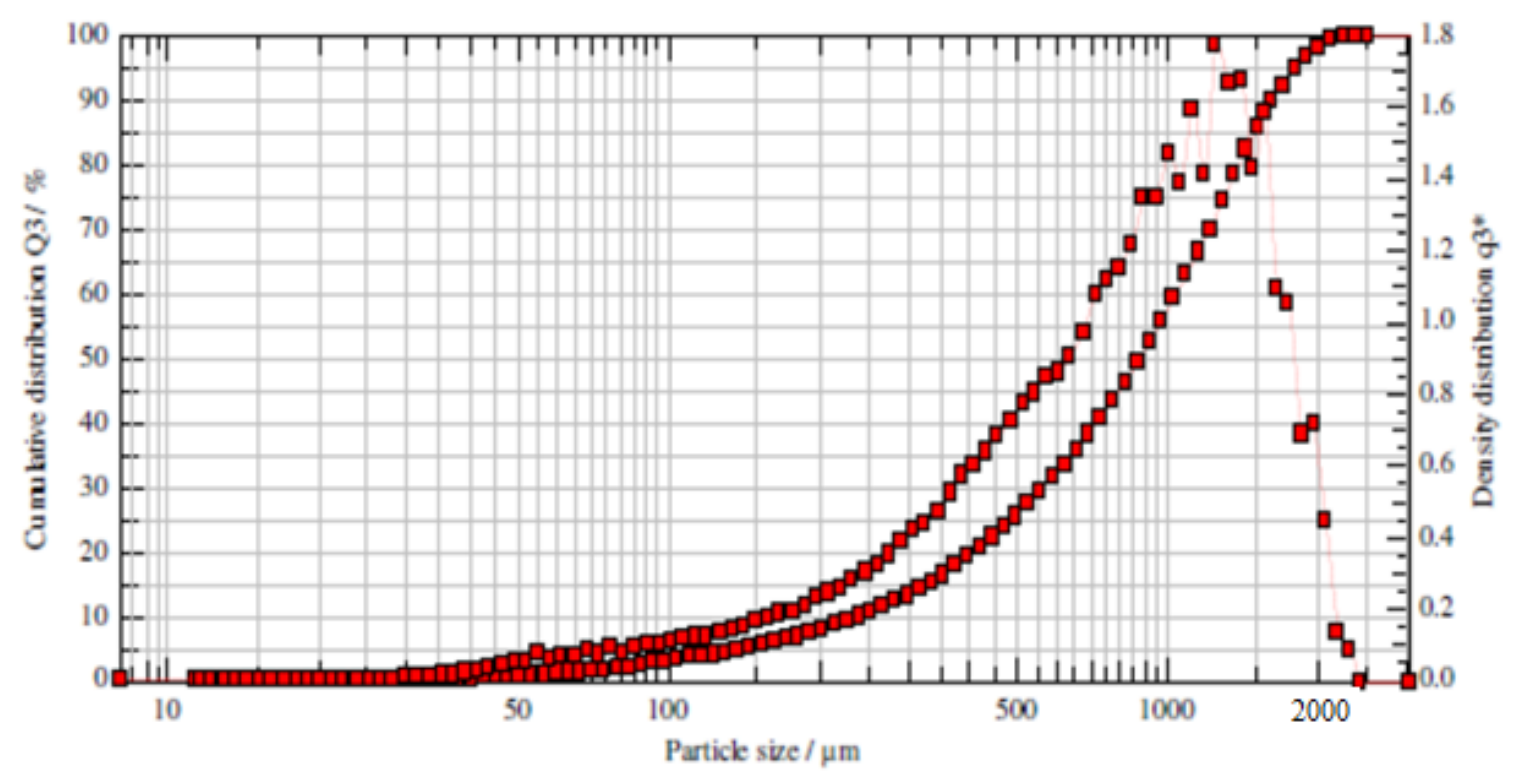

Figure 4.4 Cumulative particle size and particle size density distributions for ground flaxseed particles (Courtesy of Mr. Jonathan Tucker, U.S. DOE NETL)

Table 4.1 Properties of Flaxseed

\begin{tabular}{|l|l|}
\hline Density $\left(\mathrm{g} / \mathrm{cm}^{3}\right)$ & 2.71 \\
\hline Sphericity & 0.755 \\
\hline Diameter (based on surface area) $(\mu \mathrm{m})$ & 460.39 \\
\hline Diameter (ferret mean) & 647.76 \\
\hline Aspect ratio & 0.537 \\
\hline
\end{tabular}

Table 4.2 Experimental test conditions

\begin{tabular}{|c|c|c|}
\hline \% Solids Loading & $\begin{array}{c}\text { Mass Flow Rate of Solids } \\
\text { (lb/min) }\end{array}$ & $\begin{array}{c}\text { Mass Flow Rate of Air } \\
\text { (lb/min) }\end{array}$ \\
\hline 35 & 3.54 & 9.8666 \\
\hline 42 & 3.54 & 8.5056 \\
\hline
\end{tabular}




\begin{tabular}{|l|l|l|}
\hline 51 & 3.54 & 6.8045 \\
\hline
\end{tabular}

\section{D) High Speed Imaging of Particle Flow Fields}

High speed imaging setup and analysis of the results from experiments was mainly performed by Mr. Frank Shaffer of NETL. The experimental setup and preliminary experiments were done by WVU researchers. After the roping conditions were successfully established at the concerned loadings, high speed imaging was done and experimental findings were presented by Shaffer [37].

In order to capture the particle roping via high speed video, three Vision Research high speed cameras were setup with three orthogonal lines-of-view (perspectives). Figure 4.5 shows the orientation and lines-of-view of each high speed camera. Camera 1, a Vision Research v12.1, had a line-of-view collinear with the pipe downstream of the elbow. Camera 2, a Vision Research v341, viewed the pipe downstream of the elbow exit from below, and Camera 3, also a Vision Research v341, viewed the pipe exit from a line-of-view from the side (normal to the plane of the pipe system). Cameras 2 and 3 were synchronized. Camera 1 was set to a resolution of $400 \mathrm{x}$ 1280 pixels at 12 bit grey scale resolution at 1700 frames/sec. Camera 2 was set at $1280 \times 456 \mathrm{x}$ 12 bit resolution at 3000 frames/sec. Camera 3 was set at $2560 \times 862 \times 12$ bit at 1500 frames/sec. Figure 4.6, Figure 4.7 and Figure 4.8 show example images taken from each camera's perspective. 


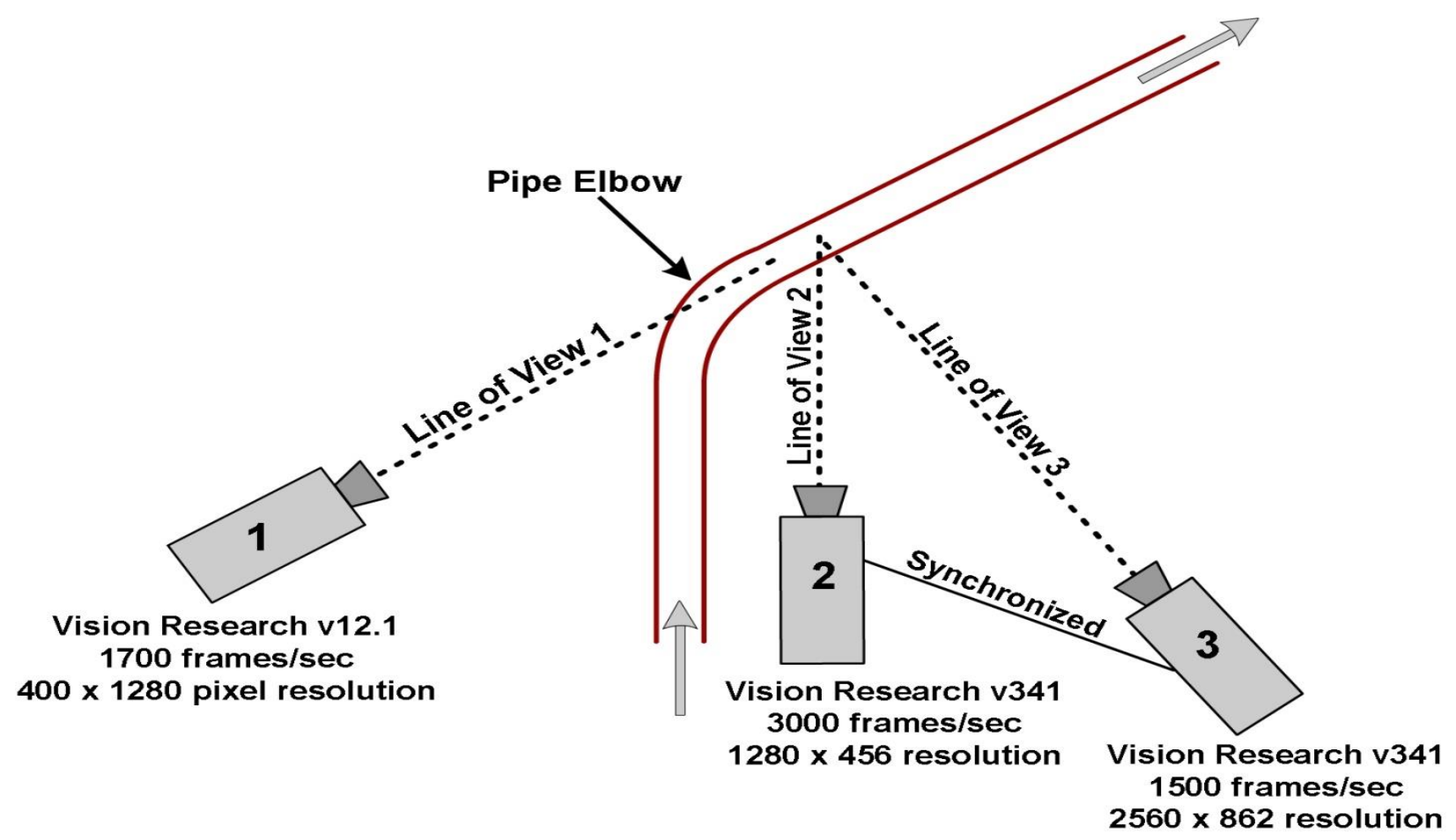

Figure 4.5 Schematic of camera perspectives for high speed imaging

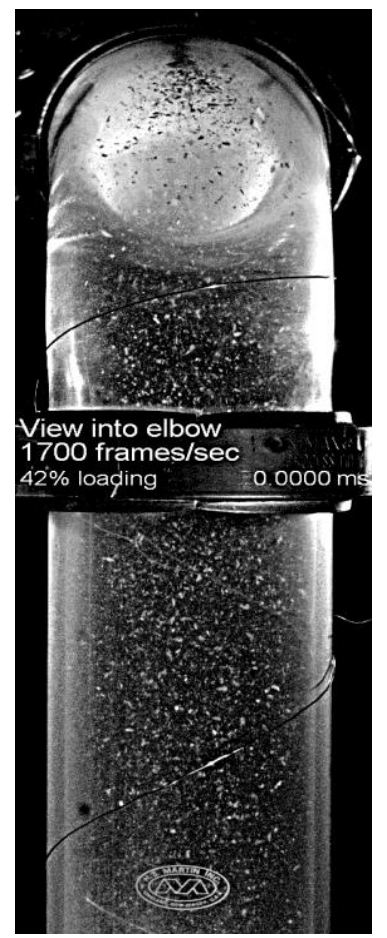

Figure 4.6 Camera 1's view aligned with elbow exit 


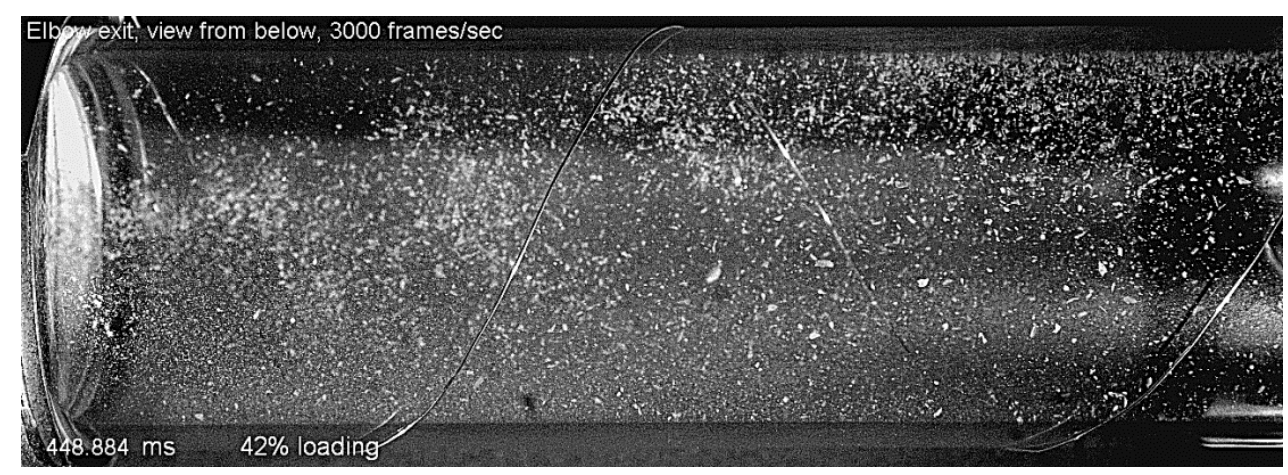

Figure 4.7 Camera 2's view of elbow exit from below

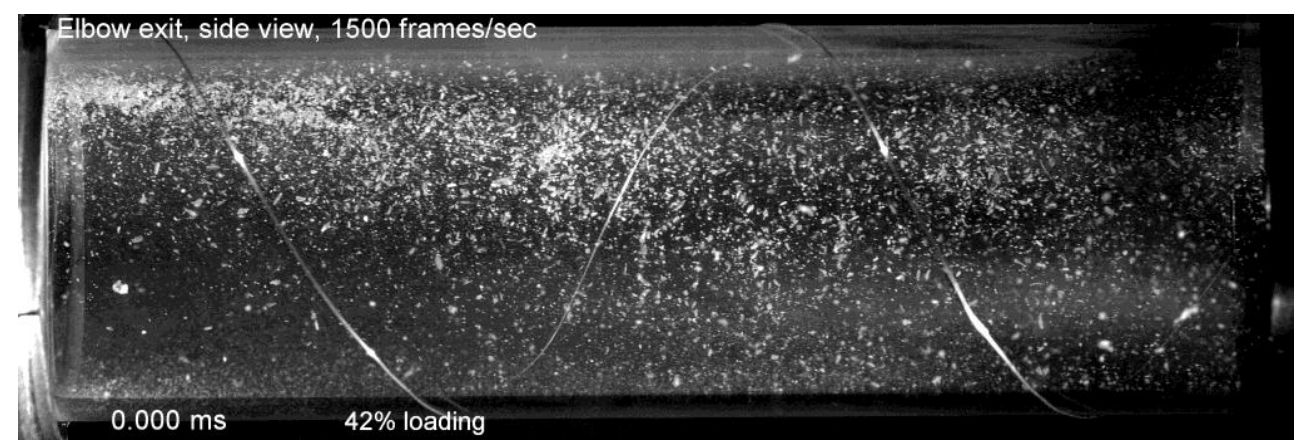

Figure 4.8 Camera 3's view of elbow exit from the side

\section{E) Measurement of Particle Concentration using High Speed Video}

Particle concentration was assumed to be proportional to the time-averaged brightness of particle images per unit area in the high speed videos. In this study, particle concentration fields are presented for the side view downstream of the elbow exit. Time averaging was done over 10,000 video frames which were recorded at 1500 frames per second, providing a total averaging time of 6.67 seconds.

To accurately measure particle concentration using high speed video, before time averaging, the effect of uneven illumination had to be removed and the particle images thresholded to render all particle images to the same brightness. The National Institutes of Health's (NIH) ImageJ image analysis suite was used for all steps in measuring particle concentration. A three step process was 
used. The first step was application of a bandpass Fast Fourier Transform (FFT) filter to remove spatial variations in brightness that were larger than the size of the particle images. The next step was to identify remaining "background" pixels (i.e., pixels with the same brightness in all video frames) followed by subtraction of the constant background brightness from each video frame. The final step was application of an Otsu threshold filter so that all particle images were rendered to the same brightness. These steps resulted in a high-speed video at 8-bit grayscale resolution with all particles images at a brightness of 255 and all background pixels at a brightness of 0 .

Figure 4.9 shows a frame from a high-speed video of the flow field exiting the pipe elbow before the image processing steps were applied. Figure 4.10 shows the same video frame after a high pass FFT was applied with a filter cutoff set at 35 pixels (the largest particle images were around 25 pixels in diameter). Figure 4.11 shows the figure after the background has been subtracted using the Image Stack Merger plugin. Figure 4.12 shows the final result after applying an Otsu thresholding filter.

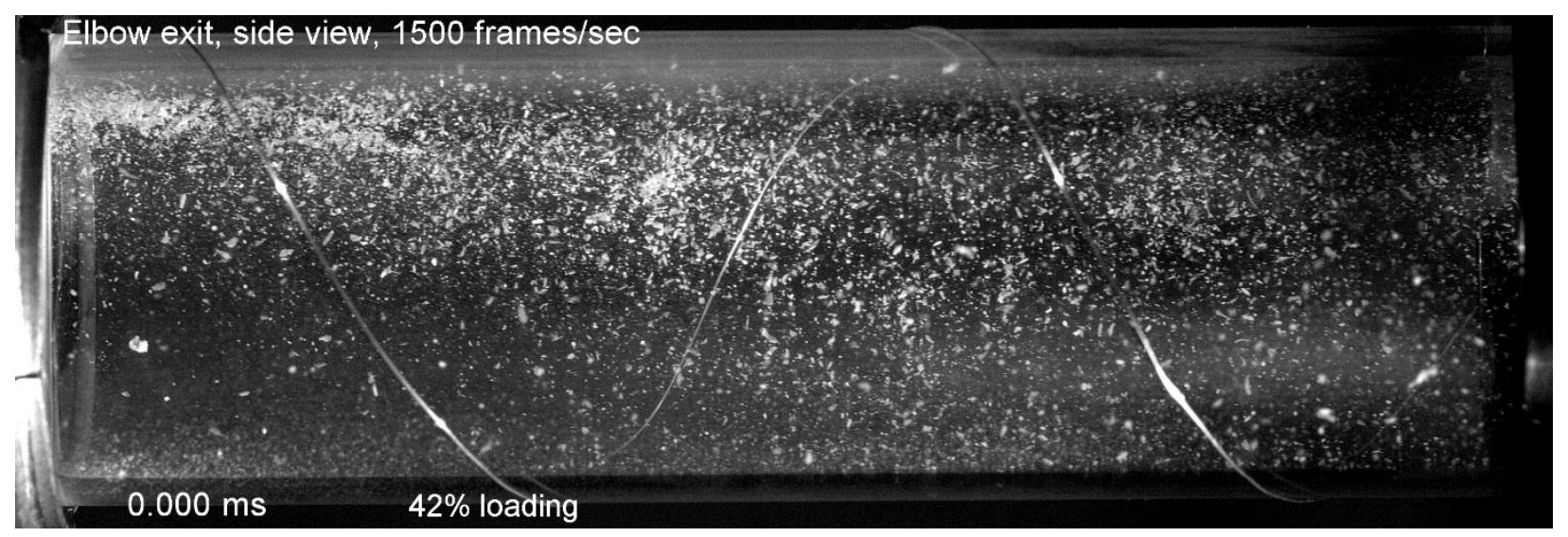

Figure 4.9 Video frame from original high speed video showing particle flow field exiting the pipe elbow. 


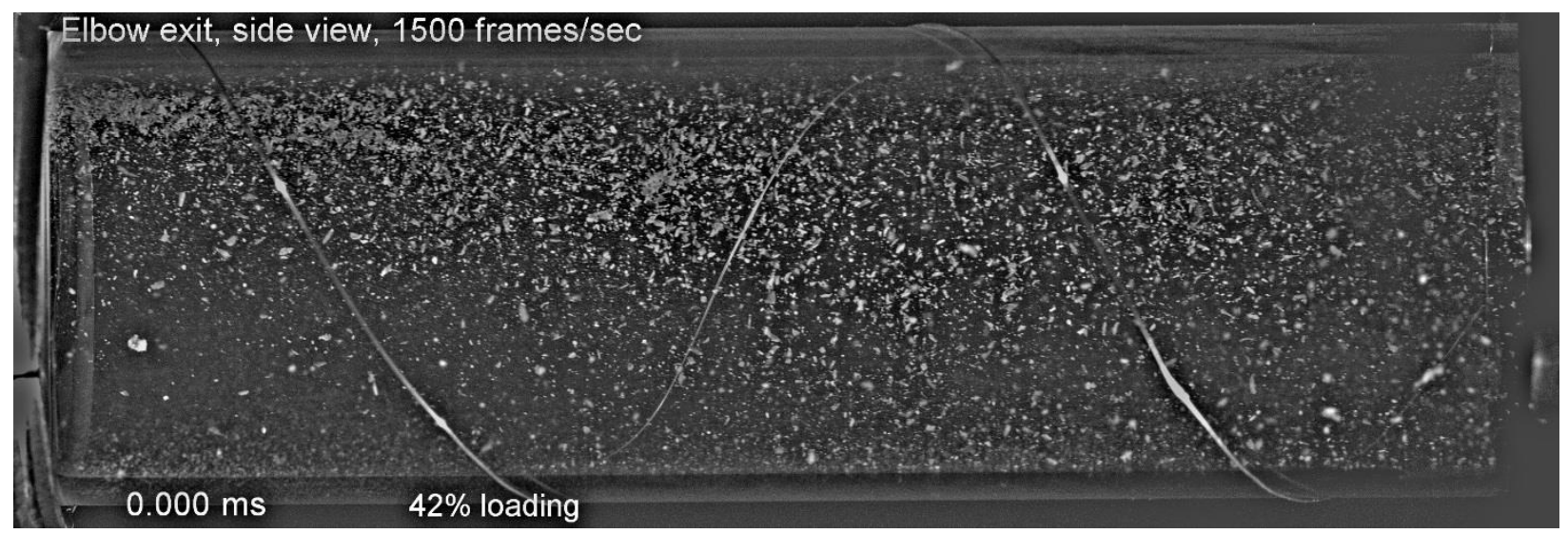

Figure 4.10 After a bandpass FFT was applied with a cutoff set at 30 pixels

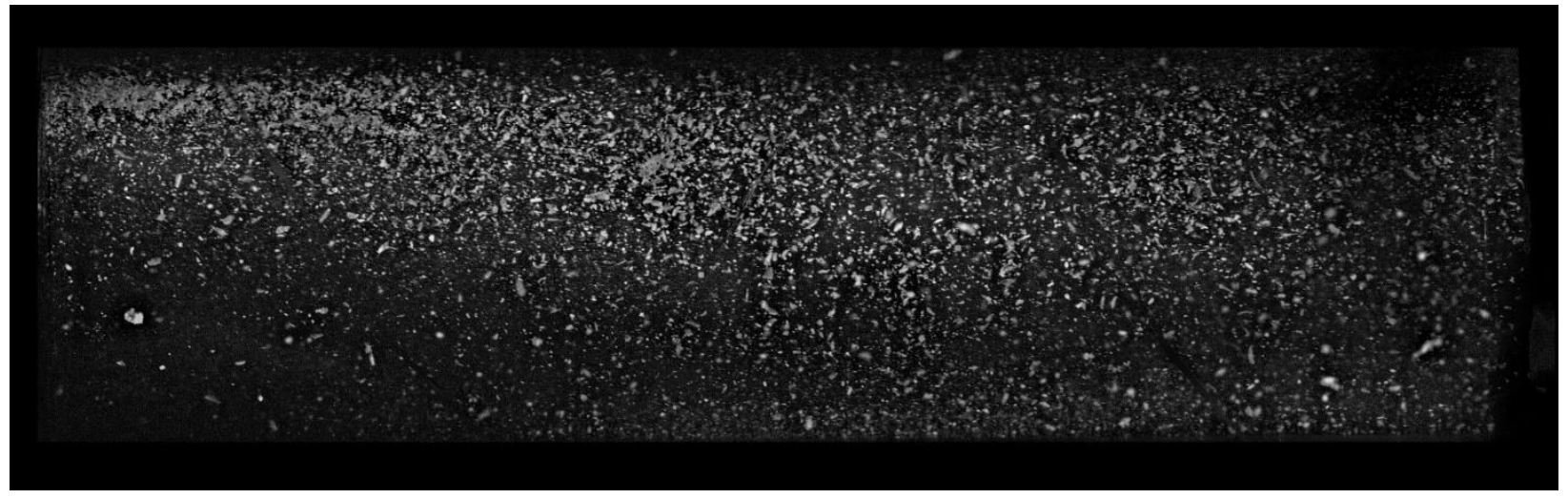

Figure 4.11 After subtracting the background image

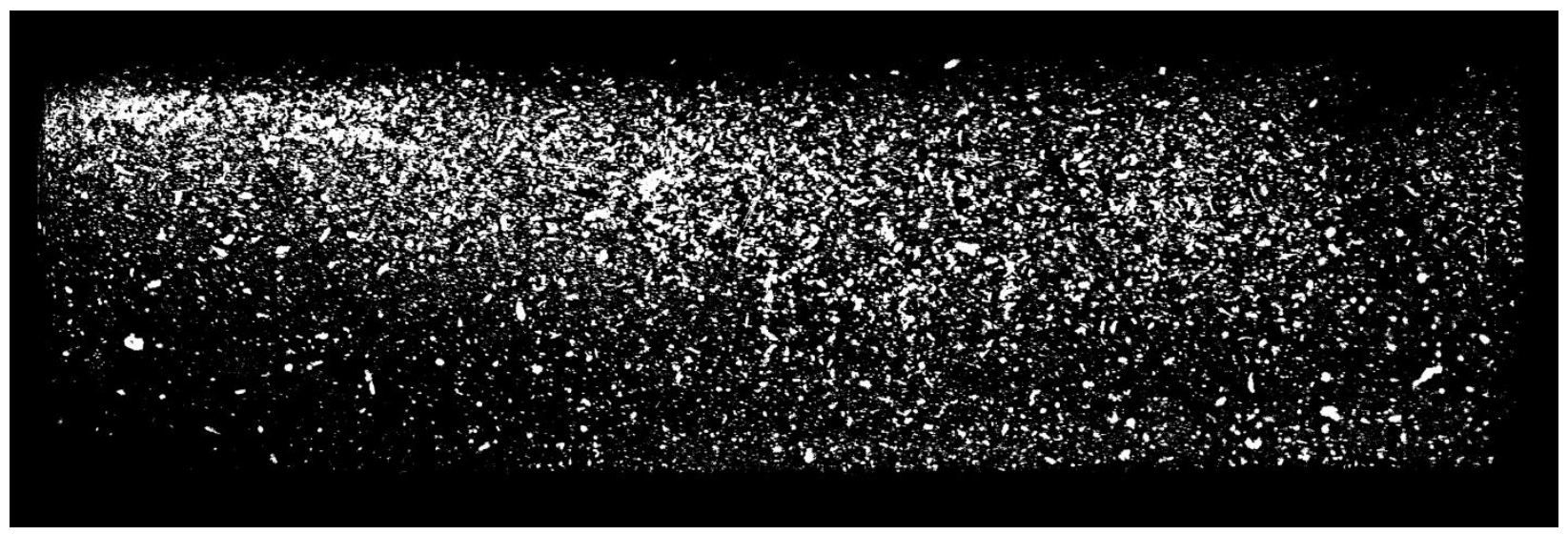

Figure 4.12 After applying an Otsu threshold filter

The concentration at any point in the video frame is normalized with the maximum concentration (i.e., the maximum brightness), which occurs in the particle "rope" when it exits the elbow at the upstream top of the elbow exit. Figure 4.13 shows the location of the maximum brightness. The 
pixels of the time-averaged frames were multiplied by a factor to set the maximum brightness to a level of 255. All concentration data have been normalized with the maximum brightness value; therefore, all concentration data has a range from 0 to 1.

\section{Point of maximum}

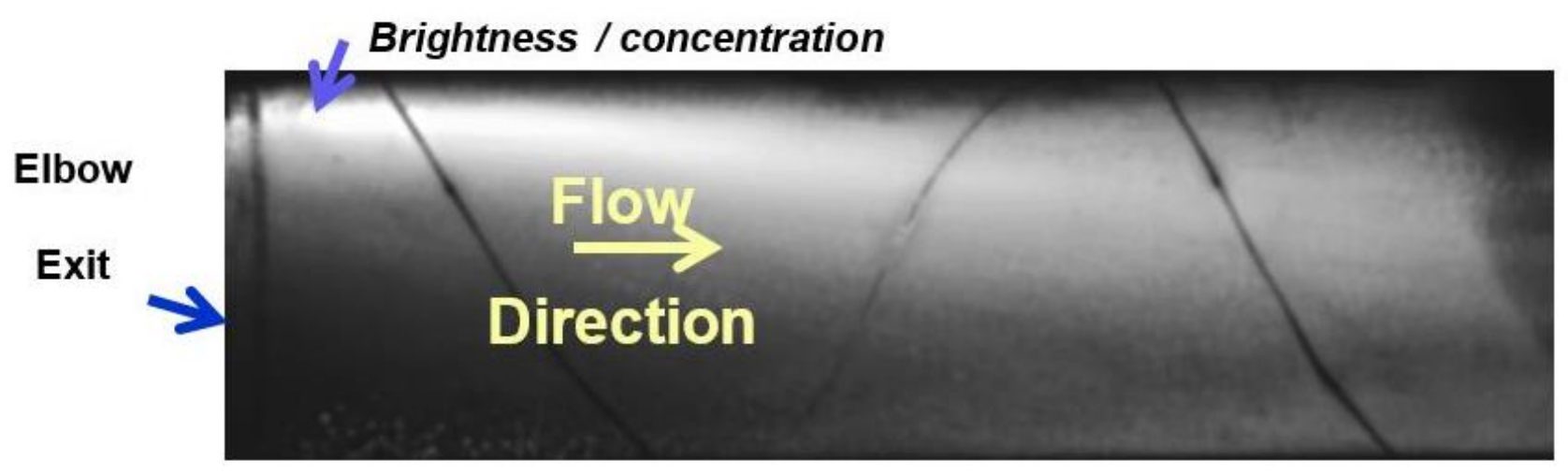

Figure 4.13 Location of maximum brightness/concentration

In this study, the particle concentration profiles have been calculated at numerous locations. Here, the three locations downstream of the elbow exit (monitor points 1,2 , and 3 ) are at $\mathrm{L} / \mathrm{D}=0.25$, 1.0, and 2.0 respectively, as shown in Figure 4.14. The particle concentration profiles were measured at these locations.

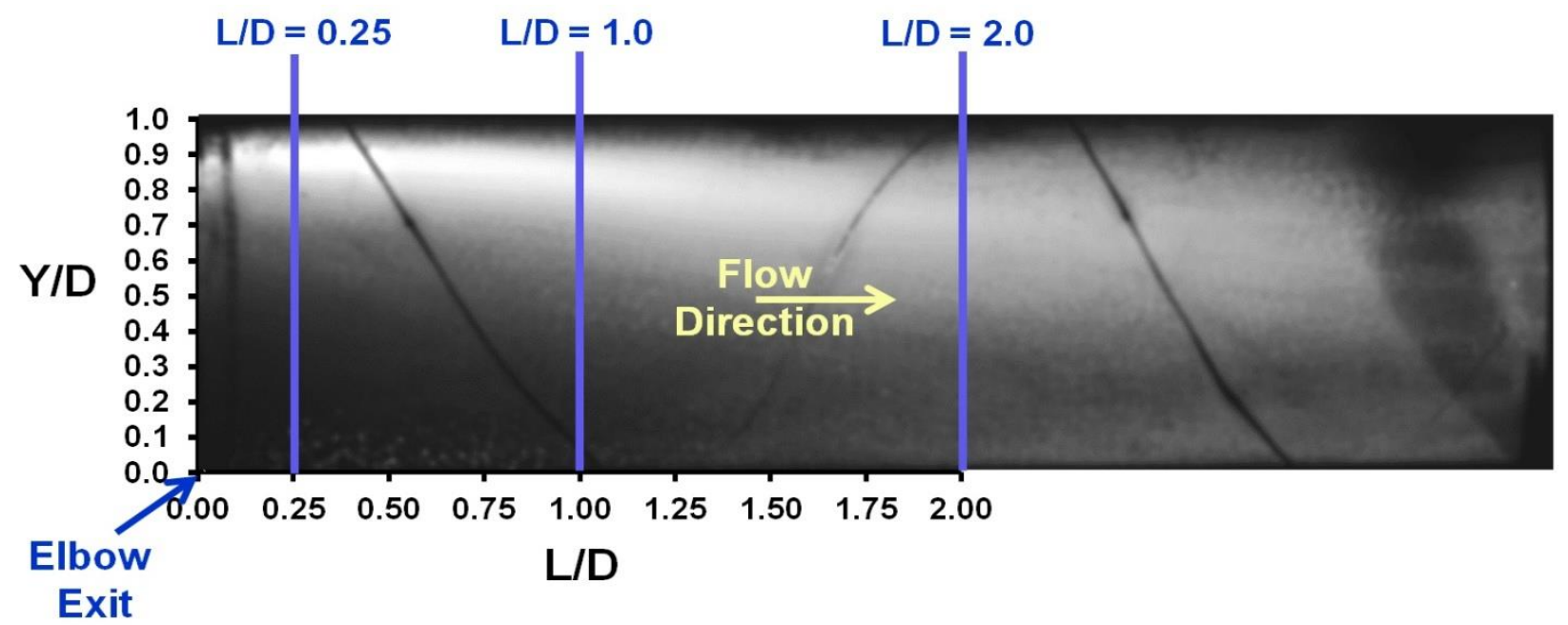

Figure 4.14 Locations downstream of elbow exit where measurements have been done 


\section{F) Challenges in Experiments}

Some challenges in conducting experiments with gas-solid flows are pulsations in the feed supply, moistness and particles sticking to surface, field of view getting obscured due to abrasive particles, static charges causing particles to scatter and loss of particles in tubing. The pulsations have been reduced by maintaining a feed rate such that flow is continuous. Also feeding system design aided this. To prevent moistness and particles sticking to the walls, the system was first run with air for a while before starting the runs to dry any moisture in the pipes. The particles were sufficiently dried overnight. Also the fine dust which mainly sticks to the walls has been sieved and removed to the maximum extent possible. Static charge was prevented by winding copper wire over the apparatus and properly grounding it. To prevent glass from getting abraded, flaxseed was used which is non-abrasive and yet demonstrates roping. Most of the particles have been reclaimed by means of a cyclone separator with very little loss in tubing for each run.

\section{G) Drawbacks in other methods and Innovation in Current Experimental Study}

Several experimental techniques such as Phase-Doppler anemometry, Laser-Doppler anemometry, some techniques with fiber optic probes and laser light sheet have been source of experimental data in many previous studies. Most of these studies [38] had an external agent introduced into the flow field to take measurements. This introduces a disturbance into the flow field and there is a chance to introduce errors in measurements and also cause changes to the flow field. With a fiber optic probe, light reflected near probe tip is the basis for measurement. Accuracy of beam spacing and focal length can affect measurements. With Laser-Doppler anemometry, the seeding particles must be small enough to follow the flow and large enough to scatter light of good quality for signal processing. Also there is a velocity bias in this technique. Higher velocities result in more detectable samples which introduces a bias. With Phase-Doppler anemometry, the main issue is it 
is a single point measuring system. To get a concentration profile, measurements have to be taken at multiple points and is time consuming.

Overcoming most of these drawbacks with the above mentioned experimental measurement techniques, high speed imaging and pixel based measurement technique has been used in this study. Issues of uneven brightness and spatial variations have been removed via three step filtering process and concentration profiles have been generated. So only the particles are taken into account for data evaluation as constant background is subtracted. This is an external measurement technique which doesn't interfere with the flow field. This technique can map the entire flow field at once for a particular view as shown in the Figure 4.15 below. So it is not that time consuming. The only challenge is that there needs to be sufficient illumination at the locations where analysis needs to be done. This has been taken care of properly in the current study. Most of the above mentioned earlier studies have used previously mentioned measurement techniques.

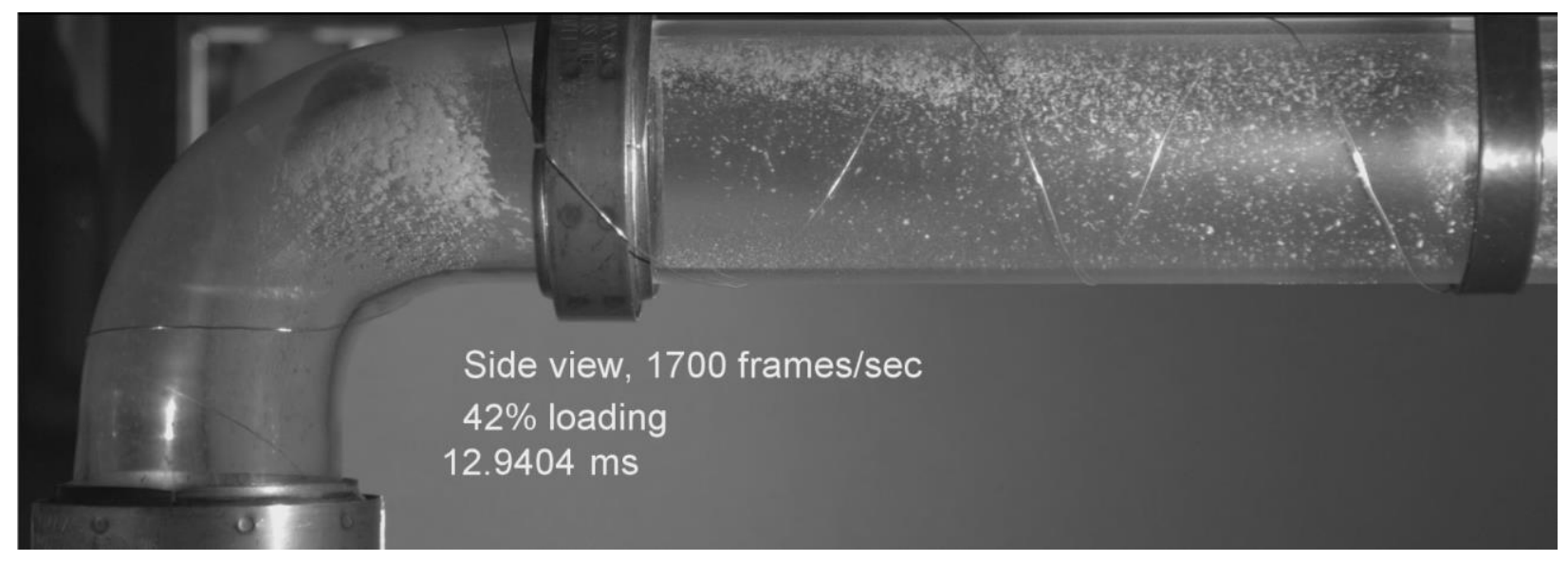

Figure 4.15 Significant portion of flow field from side view 


\subsubsection{Numerical Study of Gas-Solid Flow in Pipe Bend}

Nowadays almost every field in industry is aided by computational techniques and models for design and analysis. The power of computational models aided by experiments provide a good understanding of several phenomenon involved.

\section{A) Numerical Validation Study}

The numerical simulations in this study were performed using the commercial CFD package Ansys FLUENT. The research efforts were basically initiated by trying to replicate an established case. Numerical simulations have been performed and compared with previously published data. These validation simulations have been performed to replicate the work of H. Akilli et al. [19] for gassolid flow through a pipe bend at a mass loading of 0.3 . The RNG k- $\varepsilon$ model was used for modelling the gas-phase. The Discrete Phase Model (DPM) in FLUENT was used in this study for tracking the particles. The particle size varies from less than 45 microns to 125 microns with a mean diameter of 50 microns. The established experimental and numerical data is for coal (1680 $\mathrm{kg} / \mathrm{m}^{3}$ ) for a similar setup with 6 inch inner diameter for all the pipes and a bend radius of 3 pipe diameters. Figure 4.16 and Figure 4.17 show the concentration profiles at various locations in the horizontal pipe after the bend exit. The computed results obtained are very close to the previously published data. 


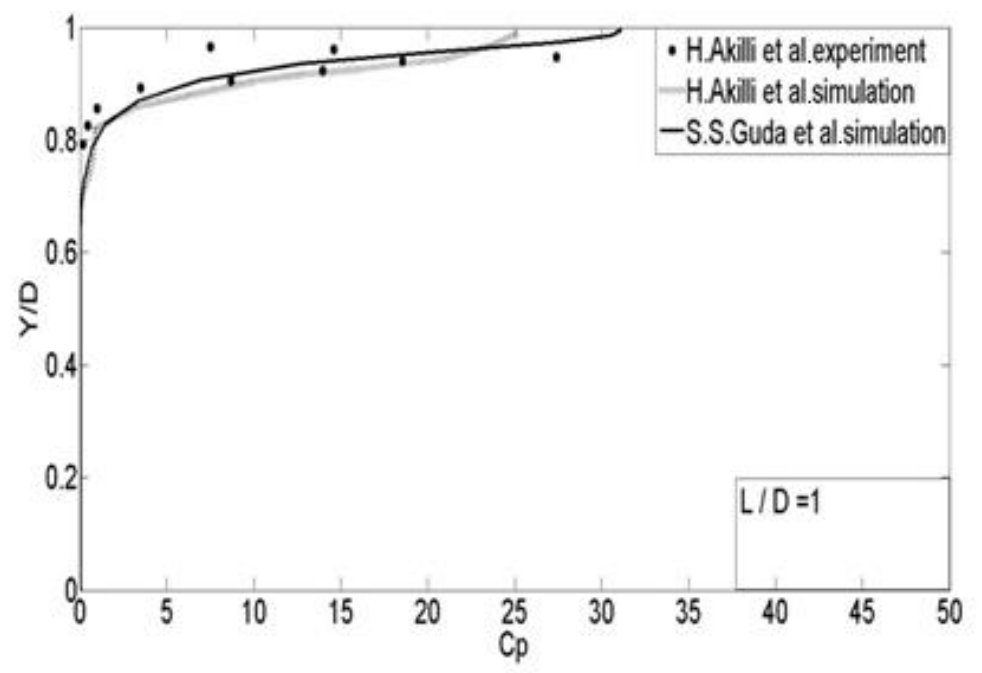

Figure 4.16 Concentration profiles at $\mathrm{L} / \mathrm{D}=1$

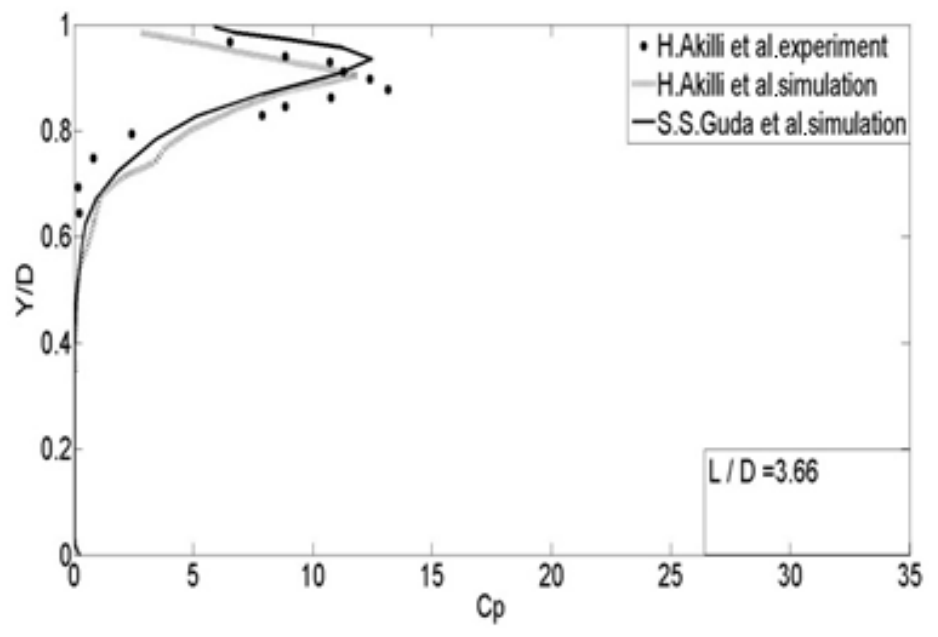

Figure 4.17 Concentration profiles at $\mathrm{L} / \mathrm{D}=3.66$

\section{B) Geometry and Mesh}

Figure 4.18 shows a side view grid of the 3D pipe bend simulated in this study. The inner diameter of the pipe is 3 inches (D). The computational models consist of a vertical pipe of 5 pipe diameters (5D), a horizontal pipe of 30 pipe diameters (30D) and a bend of radius 1.8 pipe diameters (1.8D). The hexahedral computational mesh was used in all the simulations. For the RANS simulations, a coarse mesh with 91530 cells was used. For the LES simulations, a relatively finer mesh with 579690 cells was used. 


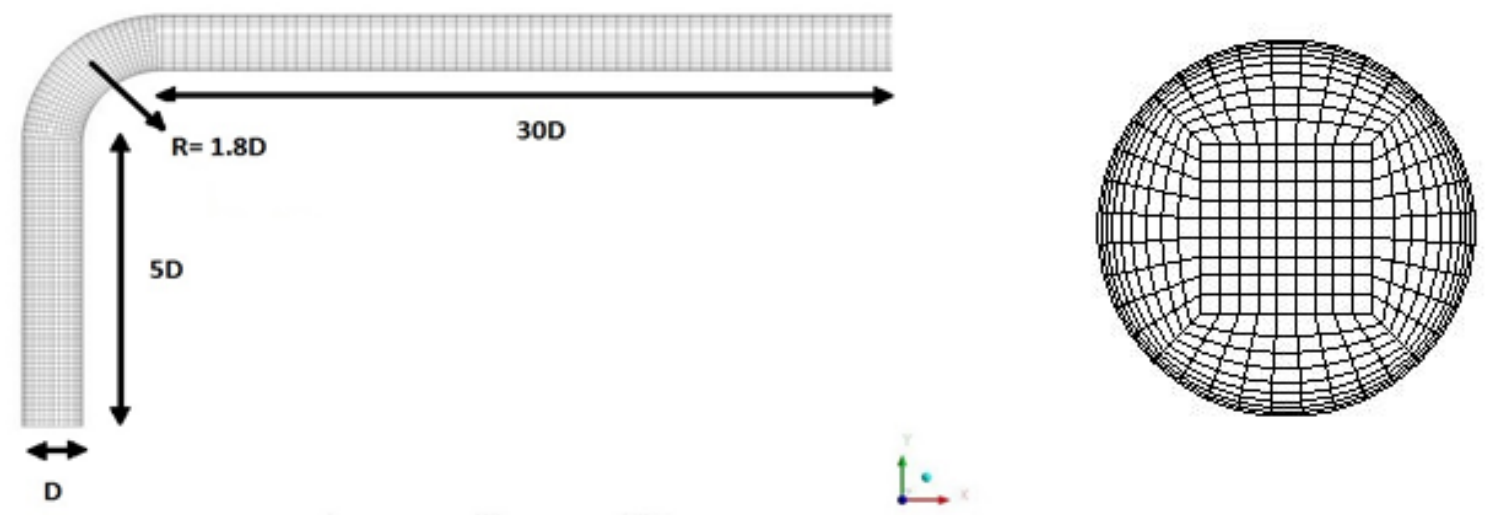

Figure 4.18 3-D coarse grid for simulations with cross-sectional view on the side

\section{C) Influence of Certain Factors on Roping in Simulations}

Roping phenomenon is influenced by several factors such as secondary flows, geometry of the bend, particle velocities, wall roughness, electro-static behavior of particles etc. Most of these have been studied in past numerical studies. Simulations have been performed to study the effect of a few other unexplored parameters. These have been performed with flaxseed using its measured size distribution.

\section{i. Effect of Loading method}

The loading condition can be varied by either changing the gas mass flow rate or by changing the particle mass flow rate. Figure 4.19 shows the comparison between simulation results in both the cases for solid-gas loadings of 35\%,42\% and $51 \%$. The profiles have been extracted from crosssectional lines from the simulation domain. The concentration profiles vary slightly but the overall pattern is similar in both the cases for all three loadings, with similar profiles but a consistent slight increase as mass loading is increased, as expected. 

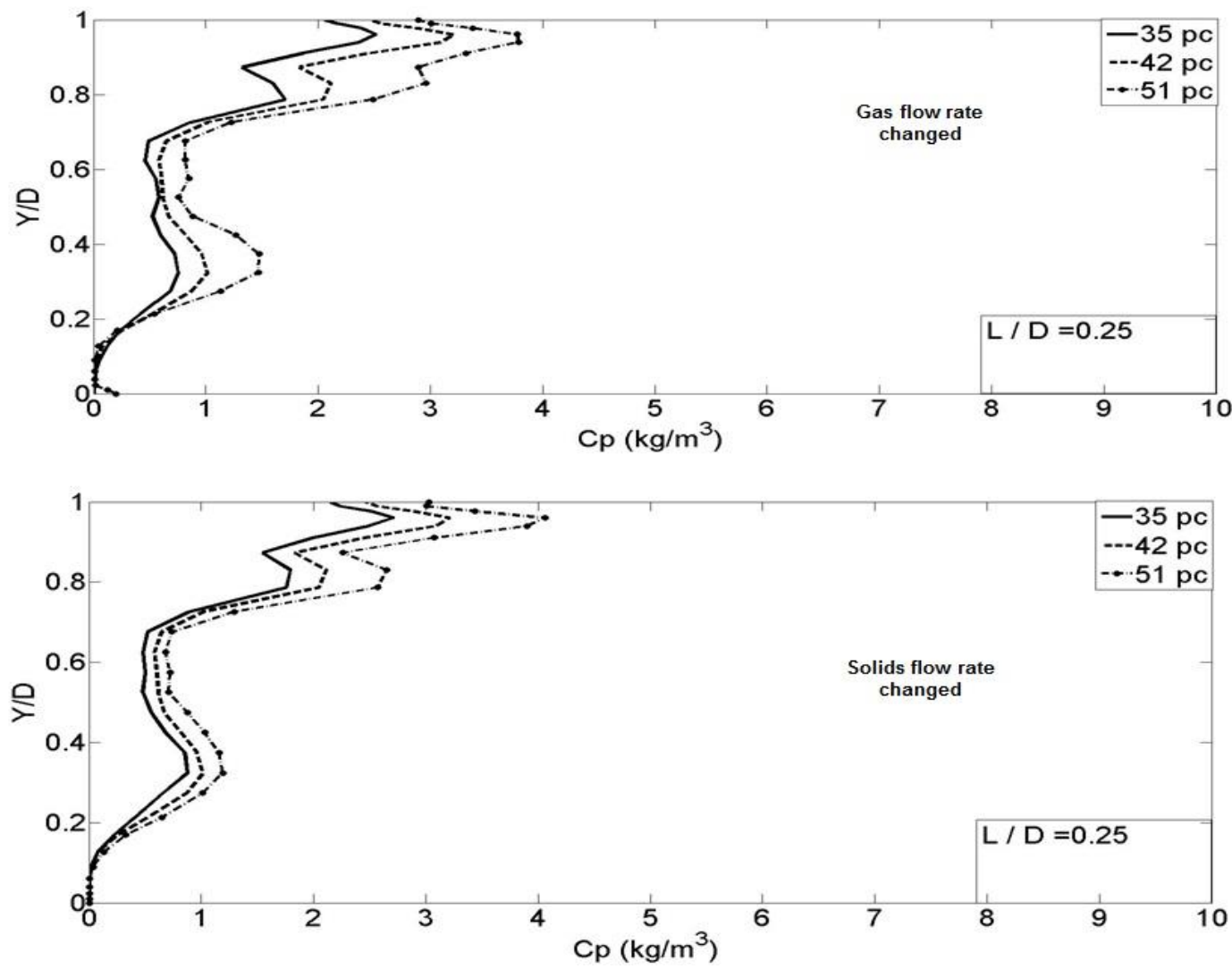

Figure 4.19 Comparison of concentrations for different methods of loading at $\mathrm{L} / \mathrm{D}=0.25$

\section{ii. Effect of Inlet Turbulent Length Scale}

The effect of variation of inlet turbulent length scale was studied at a solid-gas loading of $42 \%$. The inlet turbulent length scale was calculated and simulation was performed according to that. Simulations were performed with doubled and halved values of inlet turbulent length scale and results were compared. These are shown in Figure 4.20. There is no significant effect of change in inlet gas flow turbulent length scale on concentration profiles. 

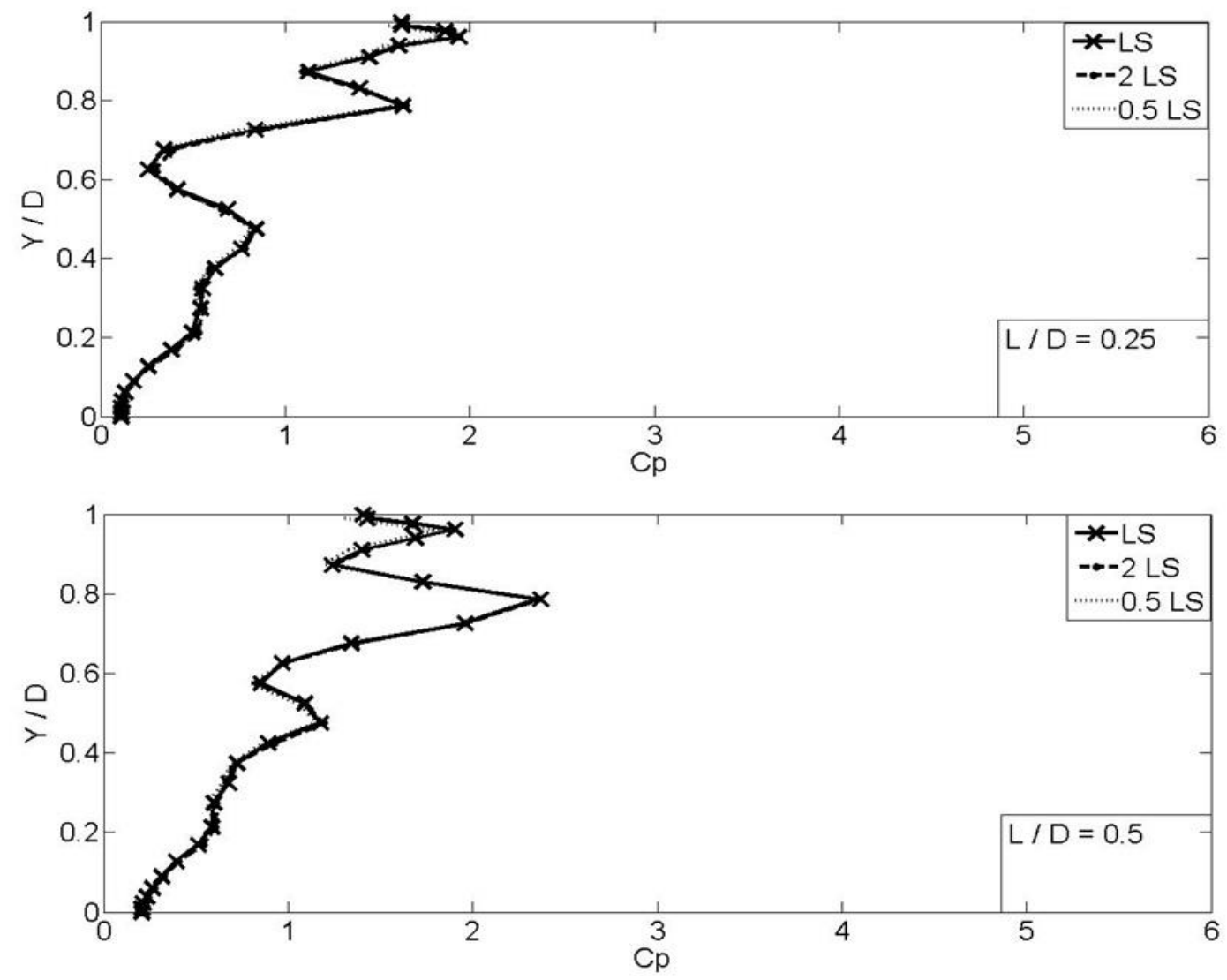

Figure 4.20 Comparison of concentrations for different inlet turbulent length scales at loading of $42 \%$

\section{iii. Effect of Inlet Turbulence Intensity}

The effect of variation of inlet turbulent intensity was studied at a solid-gas loading of $42 \%$. The inlet turbulent intensity was calculated and simulation was performed according to that. Simulations were performed with doubled and halved values of inlet turbulent intensity and results were compared. The results are shown in Figure 4.21. There is no effect of change in inlet gas phase turbulent intensity on concentration profiles. 

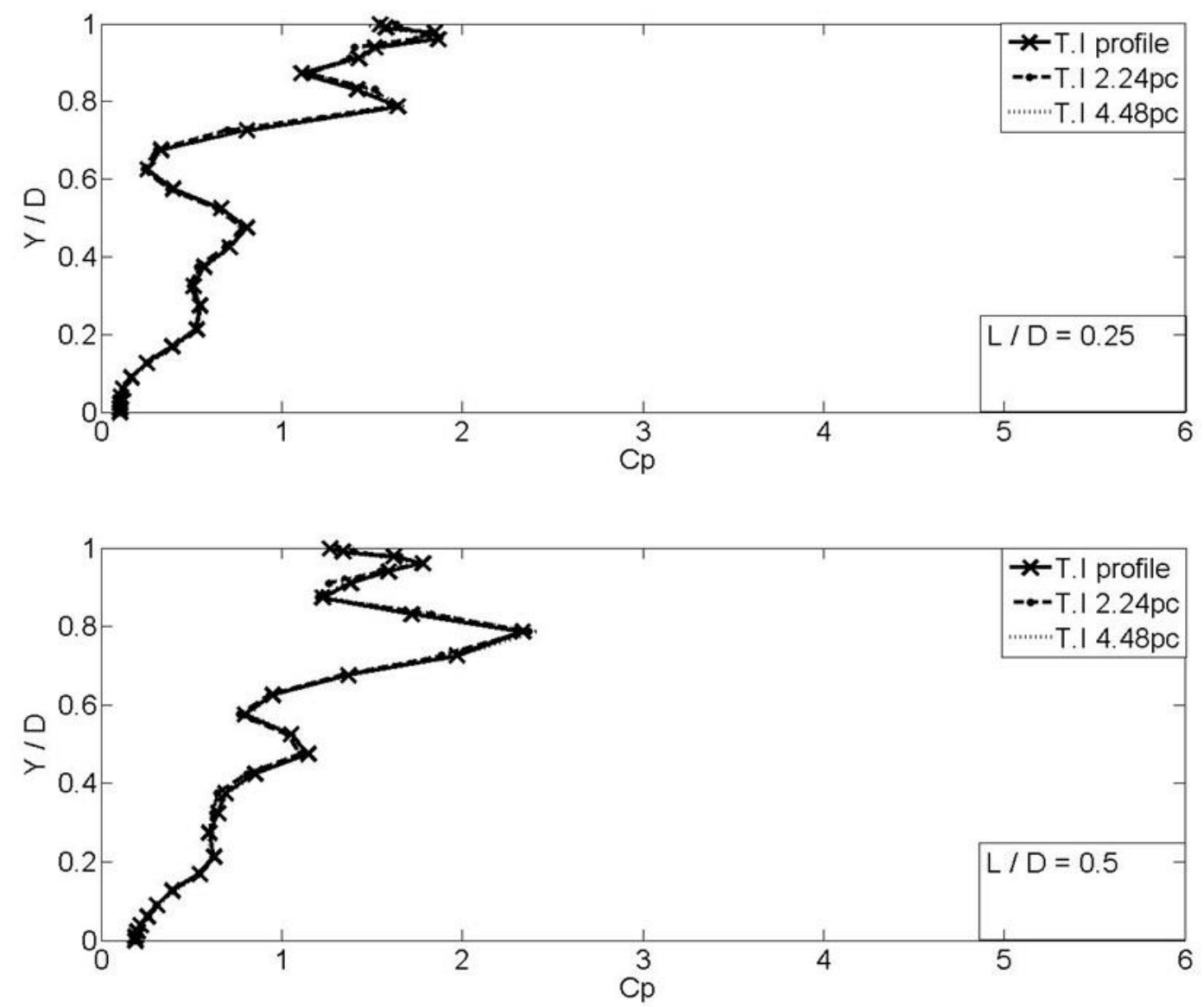

Figure 4.21 Comparison of concentrations for different inlet turbulent intensities at loading of $42 \%$

\section{iv. Effect of Particle Size Distribution and Coefficient of Restitution}

The effect of variation of size distribution and wall coefficient of restitution were studied for a loading of $35 \%$. Here results with a wide Rosin Rammler (RR) distribution of 40-2183 microns was compared to a narrow Rosin Rammler (RR) distribution of 138 -1789 microns. Also simulations were performed with coefficients of restitution of 0.9 and 0.5 . Figure 4.22 shows the results for these simulations. 


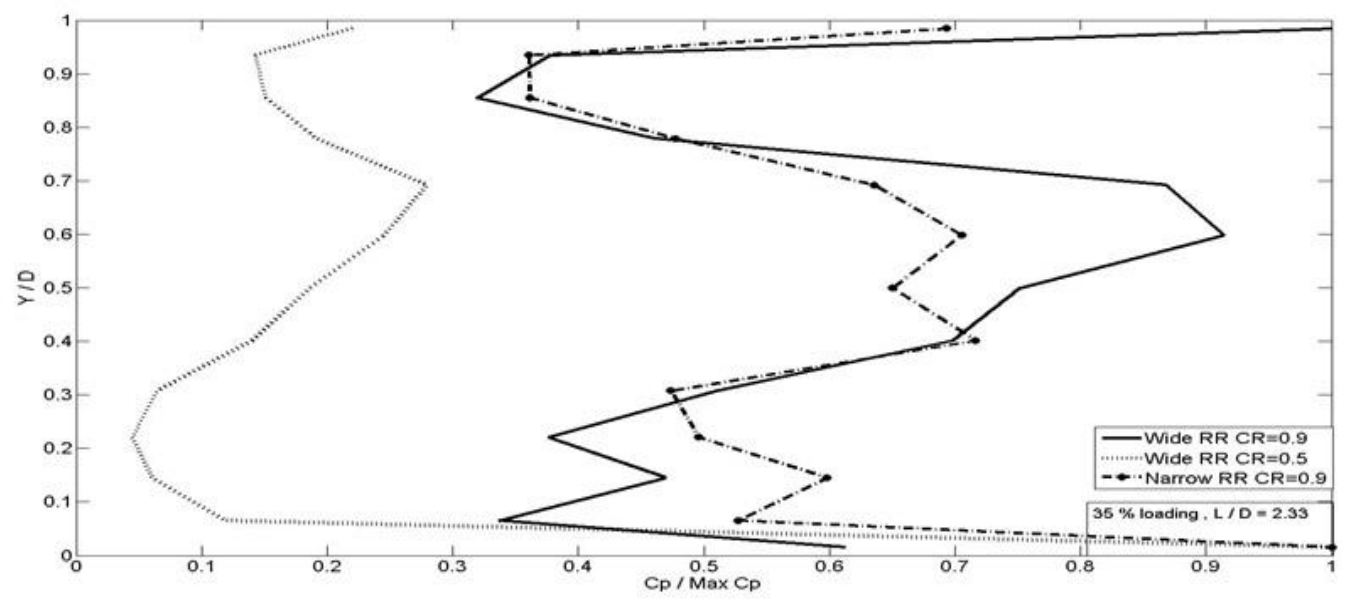

Figure 4.22 Comparison of concentrations for different particle size distributions and wall coefficients of restitution at loading of $35 \%$

Comparing the Wide RR and Narrow RR ranges for coefficient of restitution of 0.9, it can be seen that there is a significant difference in the concentration profiles at the top and bottom wall (Y/D $=1$ and $\mathrm{Y} / \mathrm{D}=0$ respectively). Since the wide range has very small particles, a lot of them still travel at the top of the pipe along with the air. We have a higher concentration at the bottom wall for the narrow range because of the presence of the large particles which go to the bottom due to gravity.

Considering the Wide RR range and comparing it for two coefficient of restitution cases, it can be seen that there is an even larger change in the concentration profile. There is a very high concentration at the bottom wall because with a coefficient of restitution of 0.5 , particles bounce back with only $50 \%$ of the initial momentum with which they hit the wall. Already the majority of the particles are slowed down due to roping phenomenon, but additionally due to this low coefficient of restitution they are further slowed down and settle down because of gravity. 


\section{D) Challenges in simulations}

One big challenge was to specify an appropriate inlet particle distribution as close to the experiments as possible. A random particle size distribution based on Rosin-Rammler distribution was specified at the inlet. The Eulerian-Lagrangian DPM simulations need good computational resources compared to the Eulerian-Eulerian and dense discrete phase simulations [39]. Being a dilute flow, the DPM model is necessary to reproduce the particle flow field in order to compare with experimental videos. Determining the coefficients of restitution for aspherical particles from experiments is not trivial. So visualization experiments were performed to narrow down onto the right set of coefficient of restitution components.

\section{E) Some Drawbacks of Previous Studies and Innovation in the Current Numerical Study}

1) In most of the previous numerical studies, a constant value of wall coefficient of restitution for particles was specified. In this study, it was determined that wall coefficient of restitution plays a significant role in capturing roping phenomenon. This effect involves both the normal and tangential components of restitution coefficient. So a basis factor $\lambda$ (ratio of tangential to normal component) was introduced which takes both components into consideration.

2) The experimental setup has several components in the feeding system. These have varying cross-sections and shapes. To replicate the experimental conditions closely in the simulations, a random particle size distribution has been generated which was specified at the inlet as not done in many previous studies [40], [41]. FLUENT has pre-defined distributions with a size bias. So they have not been used.

3) Effect of new factors such as method of loading, inlet turbulent properties were studied along with effects of particle size distribution which were not analyzed in many previous studies.

4) Several studies included a small particle size range. The distribution in this study is very wide. 


\subsubsection{Comparison of Experimental and Numerical Results}

Numerical simulations were performed using the commercial CFD package Ansys FLUENT to investigate the gas-solid flow behavior inside a pipe bend. Both RANS and LES simulations have been performed in this regard. In these simulations, the slip velocity was calculated based on the Rosin Rammler Mean diameter (RR) and Ferret Mean Diameter (FE) for the RANS cases whereas the LES simulations only used the Rosin-Rammler mean diameter. The shape factor was set to 0.755 and the coefficient of restitution is 0.9 for both normal and tangential components. A UDF has been created that generates a random location distribution of the particles at the inlet. The particle size distribution is based on the Rosin-Rammler distribution.

\section{Details of Simulated Cases}

The cases that have been simulated in the present study are summarized in Table 4.3. Simulations were performed for solid-gas loadings of $35 \%, 42 \%$, and $51 \%$ by varying the gas mass flow rate and holding the particle mass flow rate as fixed.

Table 4.3 List of simulated cases

\begin{tabular}{|c|c|c|c|}
\hline Case No. & Loading condition & $\begin{array}{c}\text { Slip Velocity Calculation } \\
\text { based on Diameter/ } \\
\text { Flaxseed size range }\end{array}$ & Turbulence model \\
\hline 1 & $35 \%$ & RR $(40-2183 \mu \mathrm{m})$ & RNG $k-\epsilon$ \\
\hline 2 & $42 \%$ & RR $(40-2183 \mu \mathrm{m})$ & RNG $k-\epsilon$ \\
\hline 3 & $51 \%$ & RR $(40-2183 \mu \mathrm{m})$ & RNG $k-\epsilon$ \\
\hline 4 & $35 \%$ & FE $(40-2183 \mu \mathrm{m})$ & RNG $k-\epsilon$ \\
\hline 5 & $42 \%$ & FE $(40-2183 \mu \mathrm{m})$ & RNG $k-\epsilon$ \\
\hline 6 & $51 \%$ & FE $(40-2183 \mu \mathrm{m})$ & RNG $k-\epsilon$ \\
\hline 7 & $35 \%$ & RR $(40-2183 \mu \mathrm{m})$ & LES \\
\hline 8 & $42 \%$ & RR $(40-2183 \mu \mathrm{m})$ & LES \\
\hline
\end{tabular}


The simulation details of the different loading conditions are listed in Table 4.4. The details of the RANS and LES simulations are shown in

Table 4.5. In all cases, the solids material is ground flaxseed with a density of $2710 \mathrm{~kg} / \mathrm{m}^{3}$ and the assumed coefficient of restitution is 0.9 .

Table 4.4 Simulation details of different loading conditions

\begin{tabular}{|c|c|c|c|}
\hline Parameter & $35 \%$ loading & $42 \%$ loading & $51 \%$ loading \\
\hline Mass flow rate of gas & $0.08097(\mathrm{~kg} / \mathrm{s})$ & $0.068(\mathrm{~kg} / \mathrm{s})$ & $0.05556(\mathrm{~kg} / \mathrm{s})$ \\
\hline $\begin{array}{c}\text { Mass flow rate of particles } \\
\text { Injection velocity of } \\
\text { particles(RR) }\end{array}$ & $0.02834(\mathrm{~kg} / \mathrm{s})$ & $0.02834(\mathrm{~kg} / \mathrm{s})$ & $0.02834(\mathrm{~kg} / \mathrm{s})$ \\
\hline $\begin{array}{c}\text { Injection velocity of } \\
\text { particles(FE) }\end{array}$ & $5.9255(\mathrm{~m} / \mathrm{s})$ & $5.604(\mathrm{~m} / \mathrm{s})$ & $3.3701(\mathrm{~m} / \mathrm{s})$ \\
\hline
\end{tabular}

Table 4.5 Details of RANS and LES simulations

\begin{tabular}{|c|c|c|}
\hline Parameter & RANS & LES \\
\hline Time step size & 0.001 & 0.0001 \\
\hline Transient Formulation & First order Implicit & Second Order Implicit \\
\hline Spatial Discretization -Momentum & Second Order Upwind & Bounded Central Differencing \\
\hline
\end{tabular}

\section{Comparison of Results}

The simulations have been performed using Ansys FLUENT with its DPM model. Three loading cases have been simulated of $35 \%, 42 \%$ and $51 \%$. The particle flow field visualizations related to experiments are primarily contributed by Yang [37]

Figure 4.23 shows the side view visualization of the rope for all the cases in the Table 4.3. The thickening of the rope and increase in the maximum particle concentration at the center of rope is clearly visible in all the cases as the loading increases. Also the general shape of the rope looks identical for the three different simulations at each particle loading condition. 


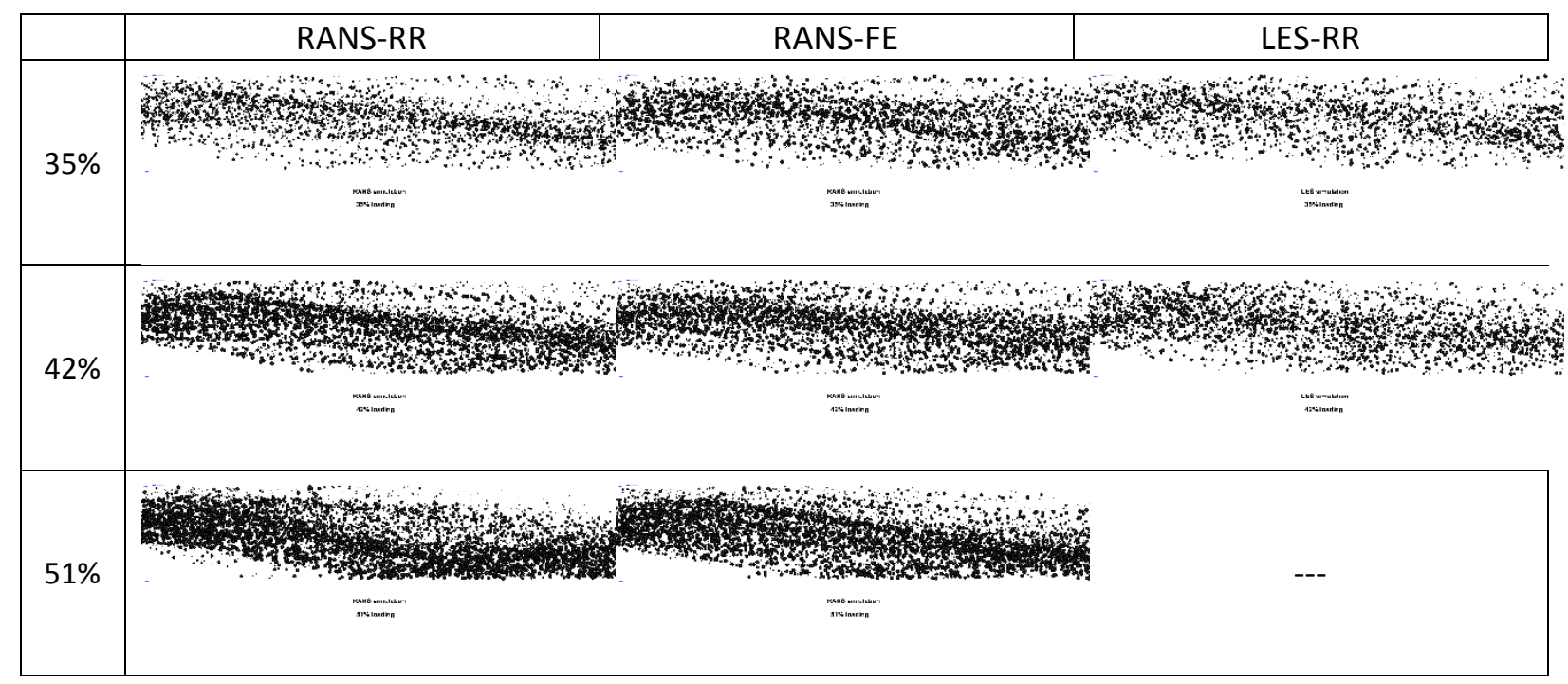

Figure 4.23 Particle visualization from side view for all cases in Table 4.3

Figure 4.24 shows the particle flow field visualization from the side view for all the simulated cases for $42 \%$ loading conditions. A very thick rope is observed in RANS as all the particles are moving towards the lower wall. The visualization from LES appears closer to the one from experiments as particles are more concentrated in the core region of the pipe. 


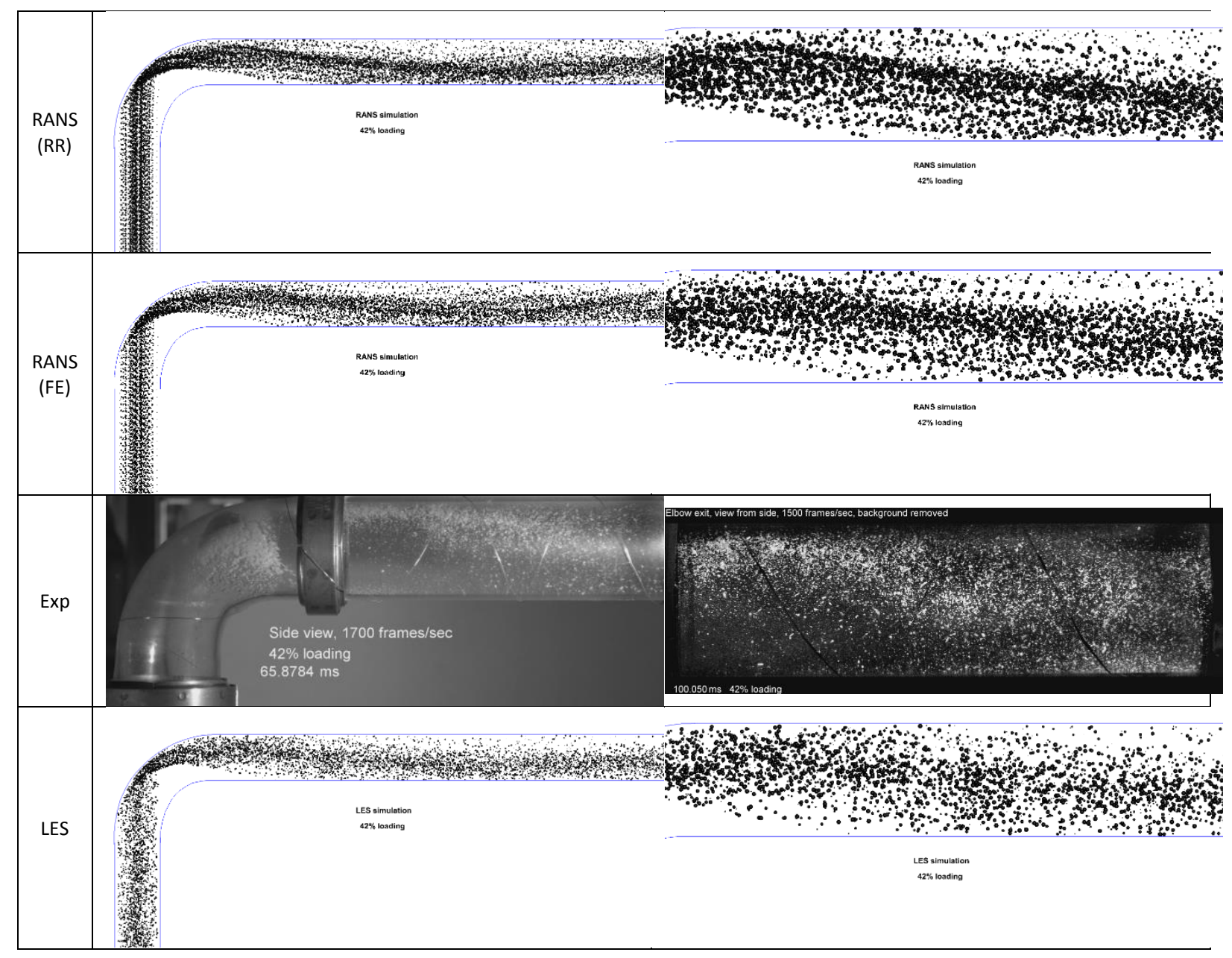

Figure 4.24 Flow visualization from side view for $42 \%$ loading

Figure 4.25 shows the particle concentration profiles along the vertical centerline of the pipe for all the simulated cases at loading of $42 \%$. The concentration profiles have been nondimensionalized by maximum bend exit concentration both in experiments as well as simulations. 
$\mathrm{L} / \mathrm{D}=\mathbf{0 . 2 5}$

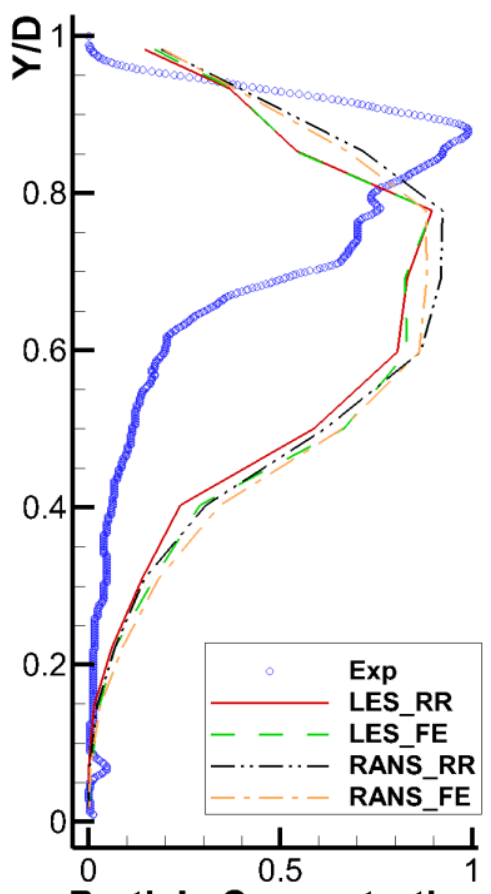

Particle Concentration
$L / D=1.0$

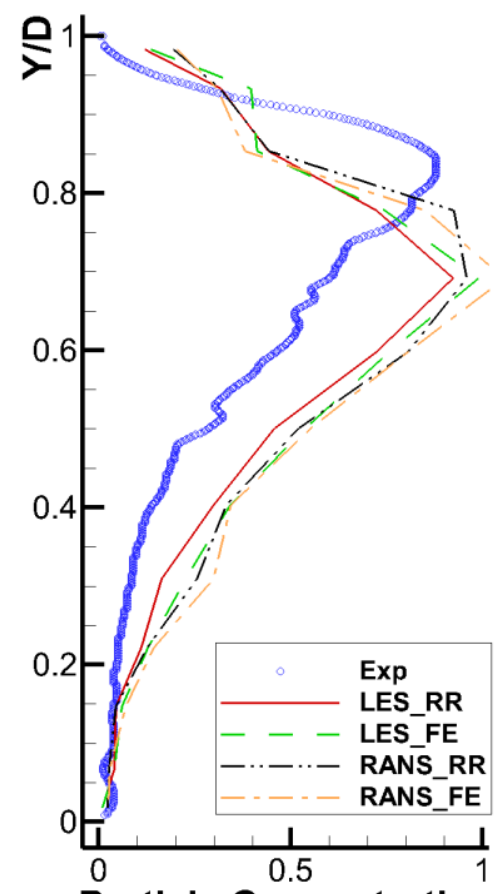

Particle Concentration
$\mathrm{L} / \mathrm{D}=\mathbf{2 . 0}$

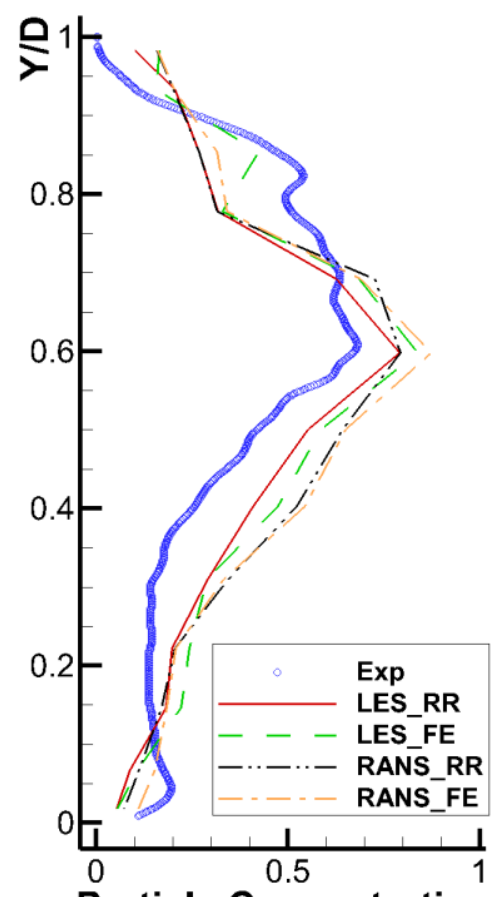

Particle Concentration

Figure 4.25 Concentration profiles for all cases at different locations for a loading of $42 \%$

The concentration profiles match reasonably well with the concentration profiles generated by pixel analysis from the high speed video. There is a slight downward shift of the peak concentration at the first two locations, while the profiles match more closely close at the third location downstream of the bend. One possible reason for this mismatch might be the unknown exact value of maximum concentration at the bend exit. If the accurate value is known, the nondimensionalization is expected to yield more accurate results. In the experiments it was observed that particles glide along the outer wall of the bend. In simulations, as the coefficient of restitution is specified as 0.9 for both normal and tangential components, the particle angle of incidence equals the reflection angle. So they deviate downward rather than gliding along the outer wall of the bend. Also concentration profiles from all cases in LES and RANS are almost similar; however 
the flow visualization is different. This may indicate that the assumed coefficient of restitution in the simulations is too high perhaps due to wall effect.

Figure 4.26 shows the particle concentration profiles for all the simulated cases at loading of $35 \%$.

$\mathrm{L} / \mathrm{D}=\mathbf{0 . 2 5}$

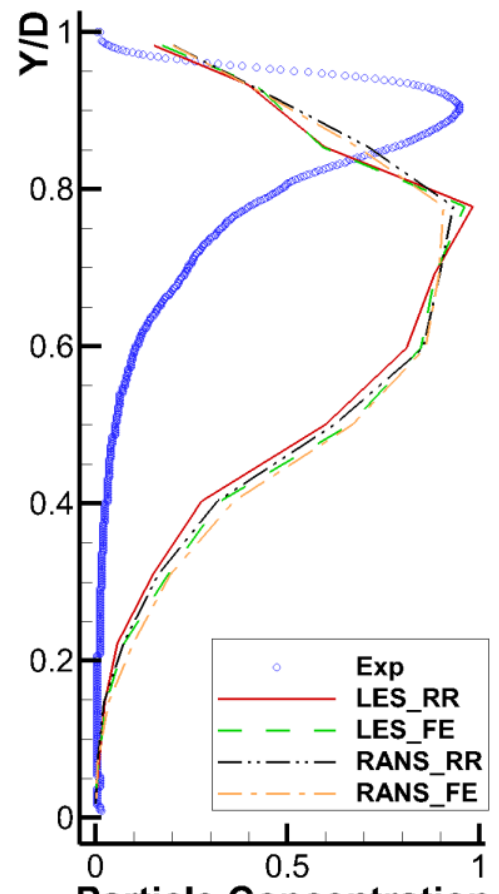

Particle Concentration
$\mathrm{L} / \mathrm{D}=1.0$

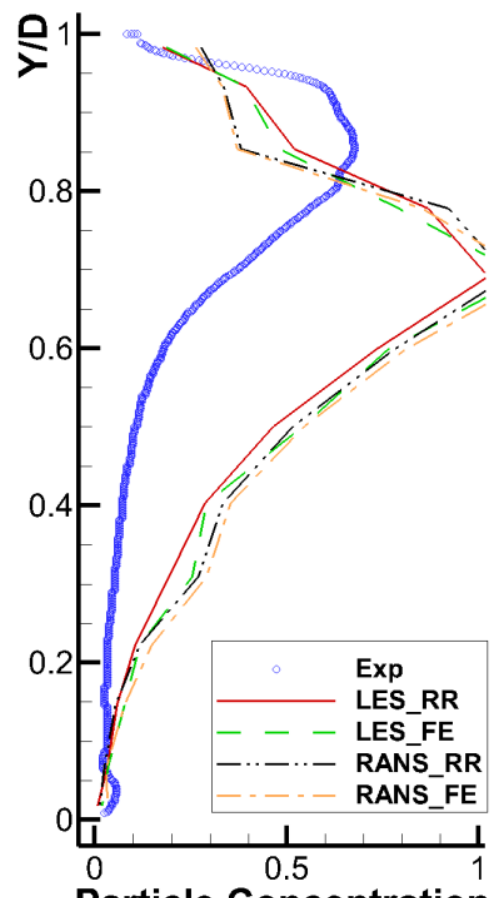

Particle Concentration
$\mathrm{L} / \mathrm{D}=\mathbf{2 . 0}$

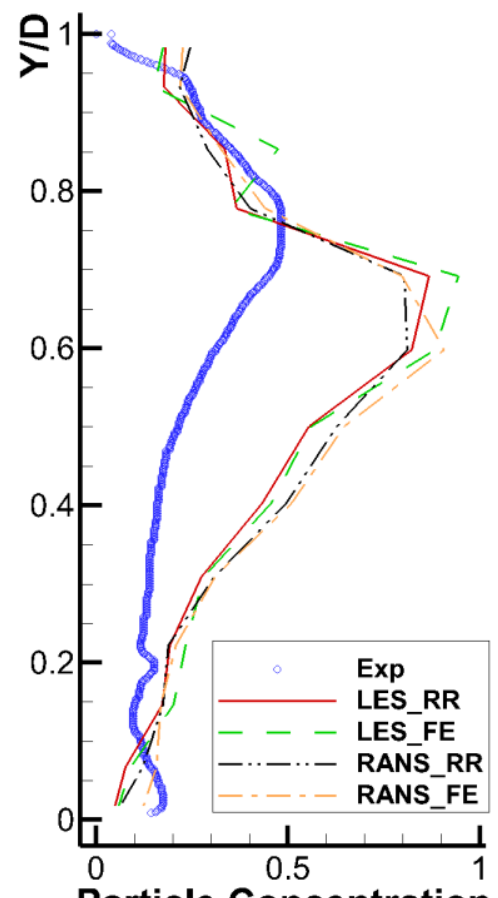

Particle Concentration

Figure 4.26 Concentration profiles for all cases at different locations for a loading of $35 \%$

It can be clearly seen from the above concentration plots that again the predicted peak concentration point is quite below that observed in experiments. Again the coefficient of restitution definitely plays a role in this discrepancy. As the coefficient of restitution is specified as 0.9 for both normal and tangential components, the particle angle of incidence equals the reflection angle. So they deviate downward rather than gliding along the outer wall of the bend. This may indicate that the assumed coefficient of restitution in the simulations is incorrect perhaps due to wall effect. 
Also another possible reason that might have led to the discrepancy is effect of inlet particle distribution. In this study, by means of a UDF, a random distribution of particles has been used at the inlet. The experimental system has several fittings like a conical distributor plate right before the vertical pipe and several tube sections leading to it. There might be effects due to these on the inlet distribution of particles. They may be scattered randomly or be biased towards a certain side in the cross-section. This effect of this needs to be quantified in the future.

Figure 4.27 shows the particle concentration contours predicted by the LES simulations at different cross-sections downstream of the pipe bend. It is observed that close to the bend exit, most of the particles are concentrated near the upper walls. These particles are then carried by secondary flows and turbulence towards the core region; and later on the rope is dissipated. The much higher peak concentration at the core region justifies the above stated behavior.

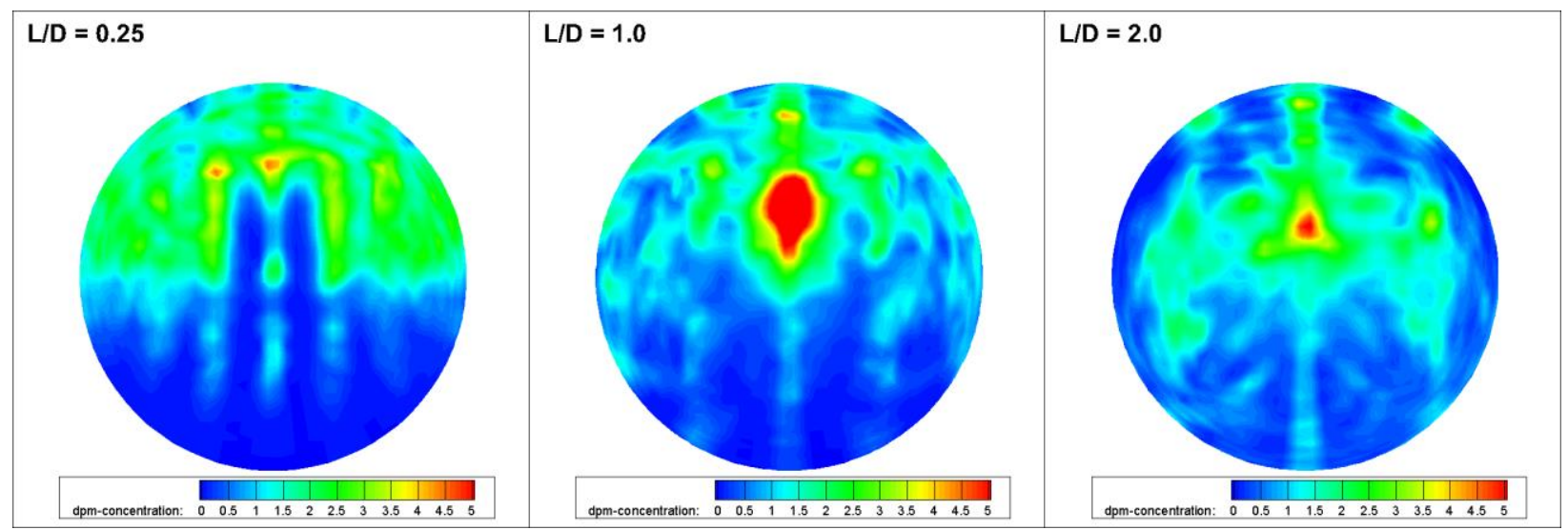

Figure 4.27 Particle concentration contour at different cross sections from LES simulations for $42 \%$ loading condition.

Figure 4.28 and Figure 4.29 show the computed axial gas velocity at different cross sections and along the pipe. The LES is expected to accurately capture eddies that carry and disperse the particles. The RANS results are more like an averaged picture. The curvature accelerates the gas 
at the top of the bend exit and the horizontal pipe as observed in literature. So higher velocity region is towards the upper wall.
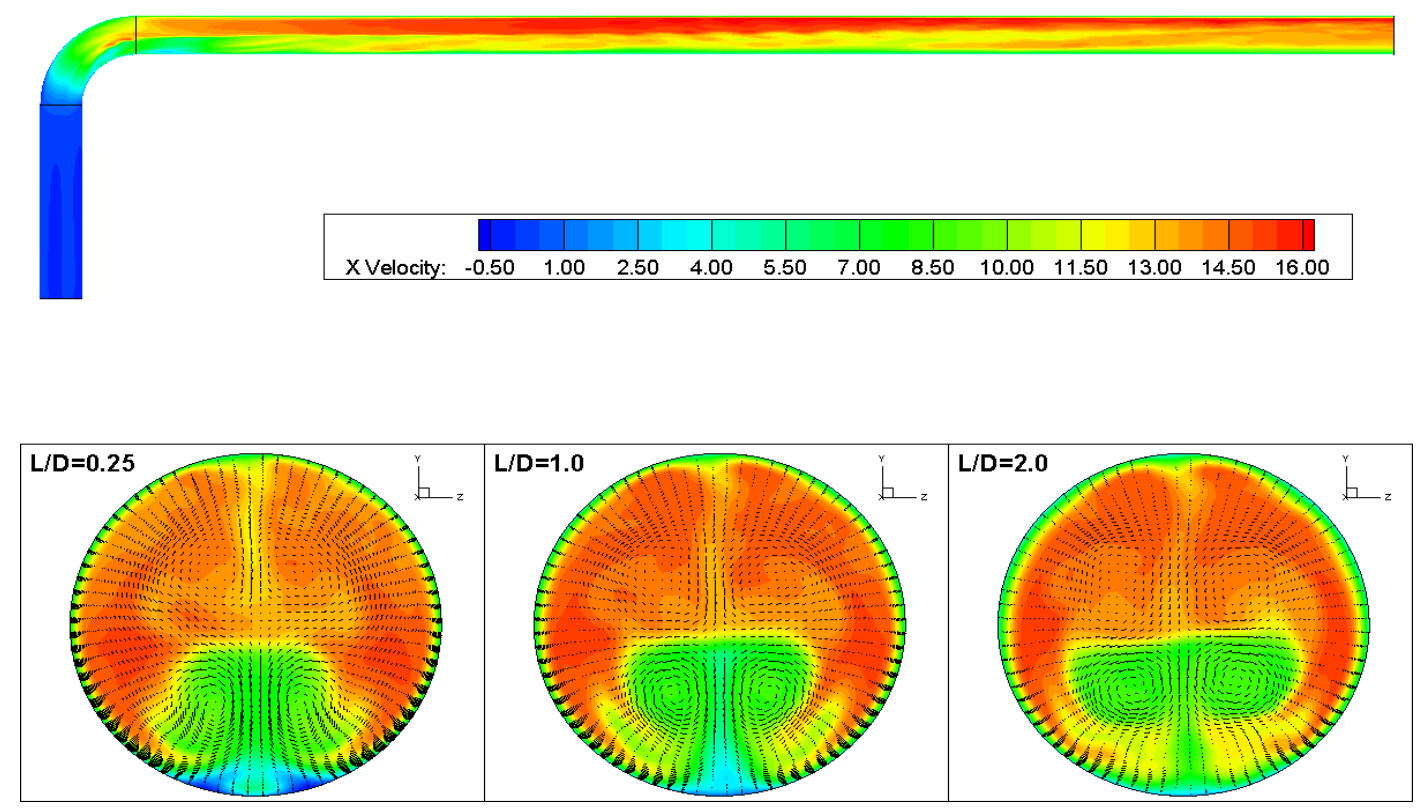

Figure 4.28 Contour plots of axial gas velocity from different views and different locations for LES simulation with $42 \%$ loading.
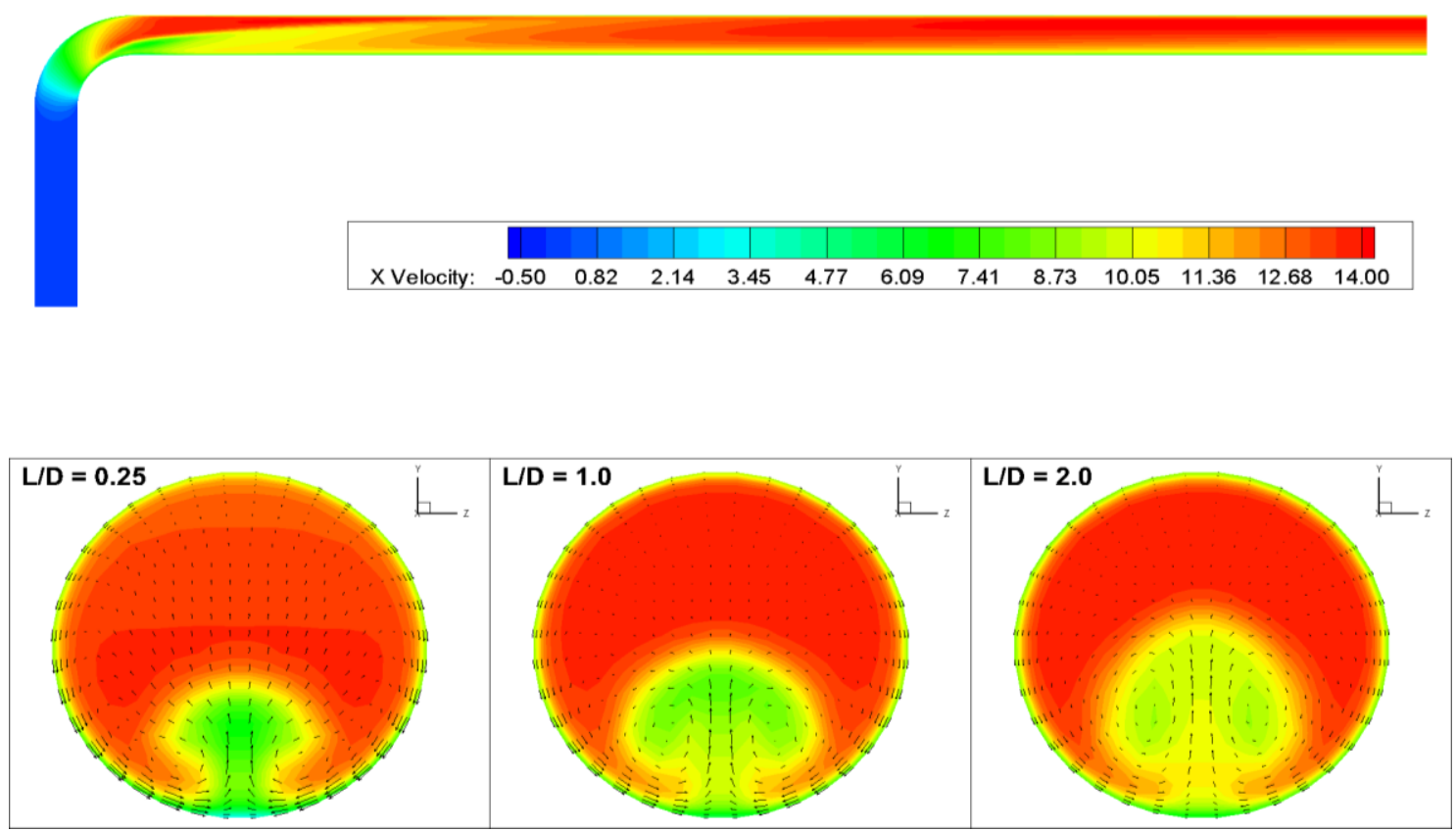

Figure 4.29 Contour plots of axial gas velocity from different views and different locations (RR) RANS simulation with $42 \%$ loading. 


\section{Effect of Coefficients of Restitution}

The restitution coefficient determines the reflection angle and momentum exchange/loss when solid particles collide with the wall, especially the elbow section. Aspherical particle shape produces a variation in restitution coefficient, with a time-averaged mean value and variation around the mean [42]. Although experimental measurement of restitution coefficient for aspherical particles is difficult [42], it is a required input in CFD models. The effects of restitution coefficient are also investigated in the present study. RANS simulations were performed for $42 \%$ loading cases with various values of $\lambda$, defined in Figure 4.30 where $\lambda$ is the ratio of tangential component to the normal component of coefficient of restitution.

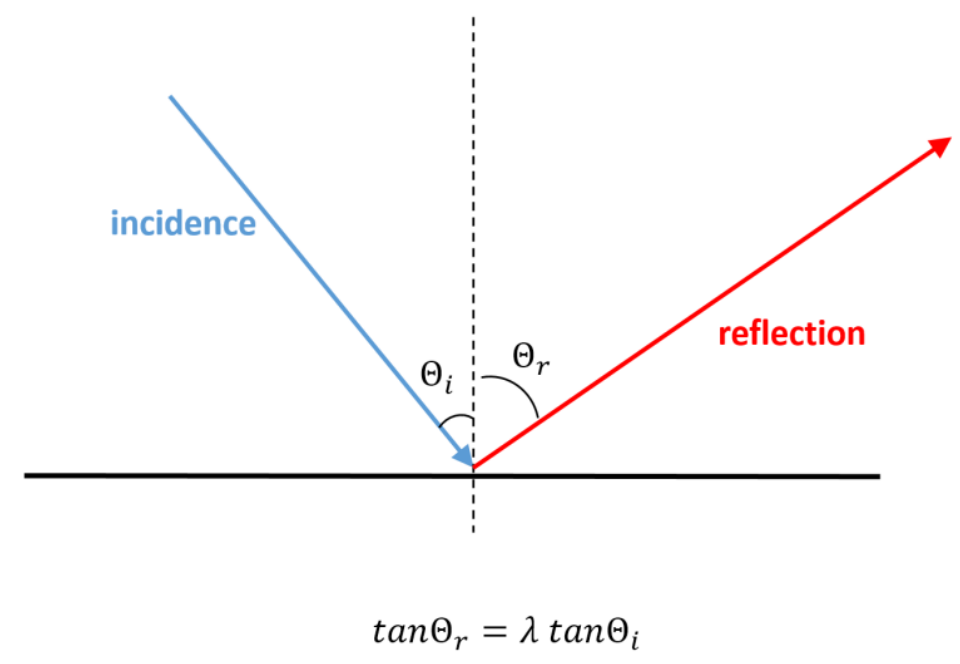

Figure 4.30 Definition of parameter $\lambda$ in the study of restitution coefficients.

Figure 4.31 shows the effects of $\lambda$ on the calculated gas-solid flow. It can be observed that the particle concentration profiles are very sensitive to restitution coefficients.

This conclusion can also be confirmed by particle visualization shown in 
Figure 4.32. The results show that without having an accurate value for $\lambda$ the roping phenomena cannot be predicted. Thus, we employed the visualization experiments to determine an approximate $\lambda$ value, 1.3, for the present used solids material.
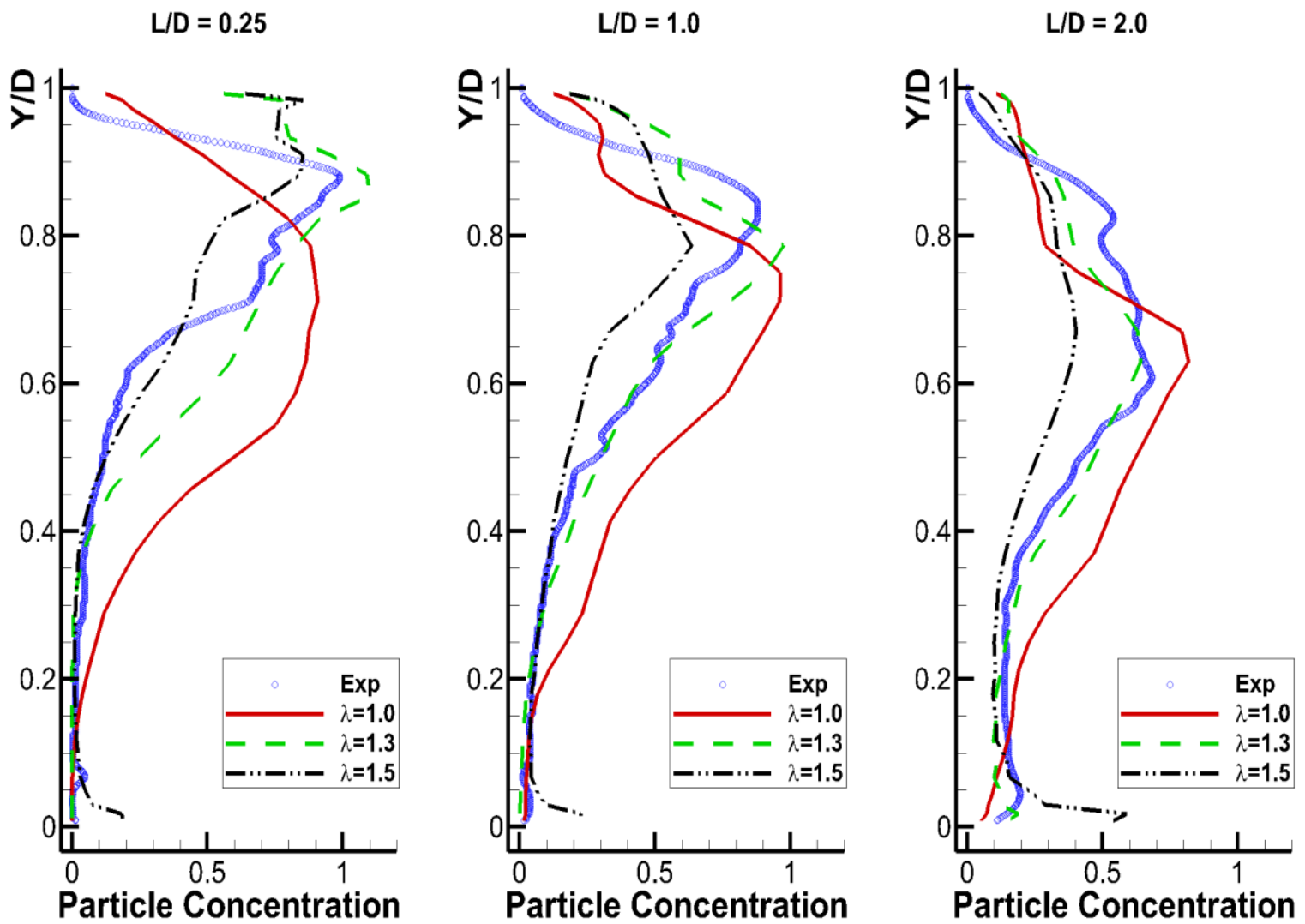

Figure 4.31 Particle concentration profiles from experiments and the RANS simulations for $42 \%$ loading condition with various values of $\lambda$. 


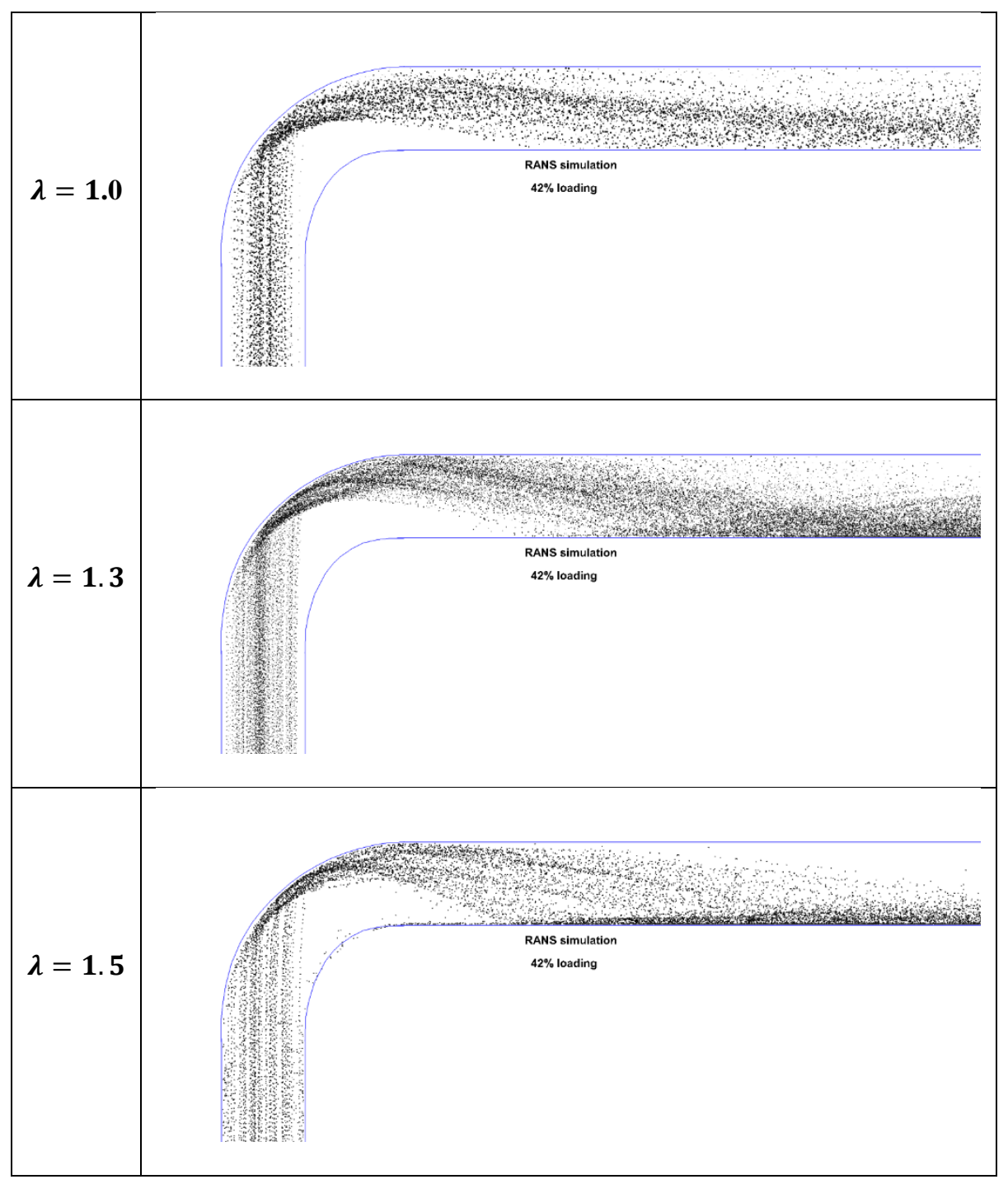

Figure 4.32 Particle visualization figures from the side view for RANS simulations for $42 \%$ loading condition with various values of $\boldsymbol{\lambda}$.

The same set of simulations have been repeated for the loading of $42 \%$ but with changed coefficients of restitution. The normal component has been set to a value of 0.692 and tangential 
component has been kept at the original value of 0.9 , the ratio of tangential component to normal component being $\lambda=1.3$. The RANS and LES simulations have been repeated with these changes. Figure 4.33 shows the comparison of concentration profiles at different locations for loading of $42 \%$.
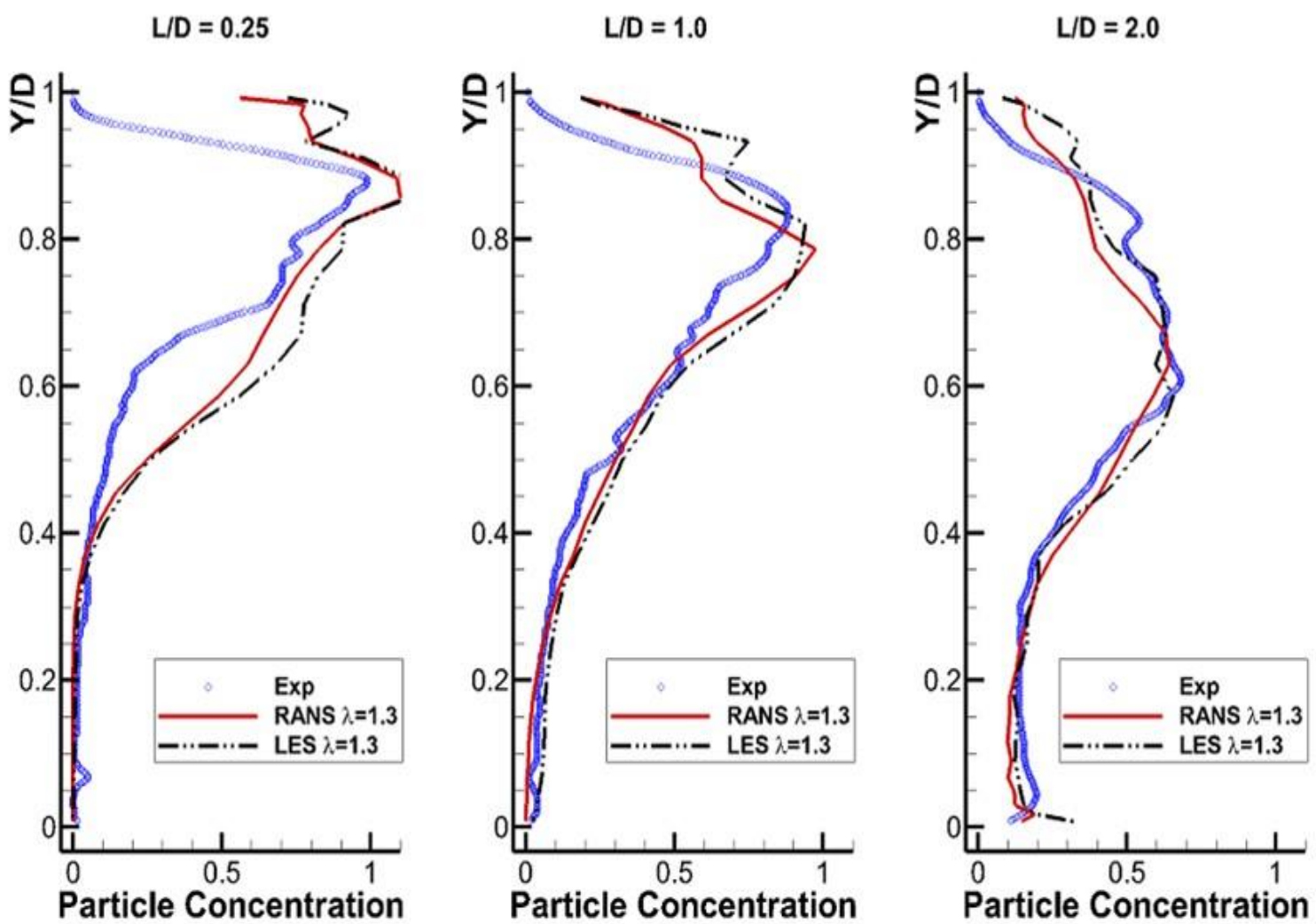

Figure 4.33 Concentrations at different locations for loading of $42 \%$ with $\lambda=1.3$

The concentrations from these simulations match extremely well with ones from experiment for both the simulations at all three locations. The maximum concentration peaks appear at the same level and also rope dispersion matches quite well. There is definitely a significant effect of the assumed value of particle coefficient of restitution on the roping phenomenon. Figure 4.34 shows a comparison of the particle flow field visualization from the side view. The LES simulation shows 
a nice and clear rope as observed in experimental videos. The RANS results are good, but the well pronounced rope is more evident in LES.

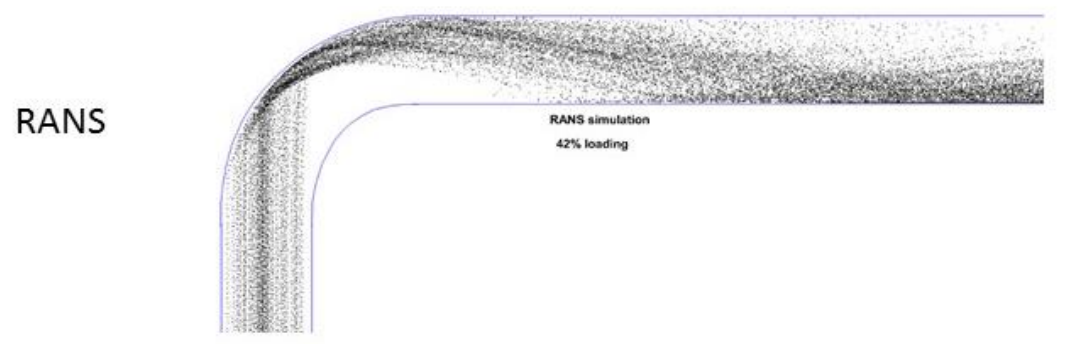

EXP

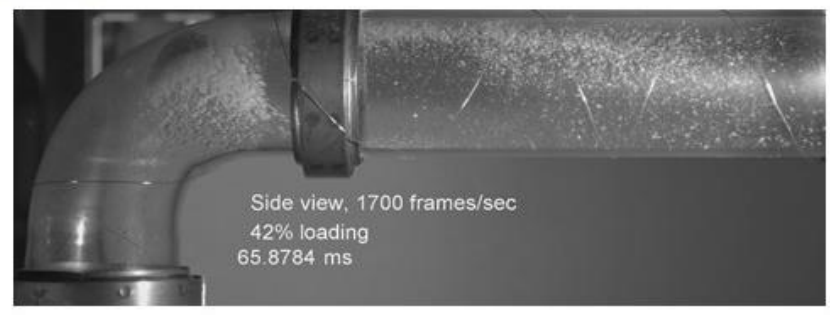

LES

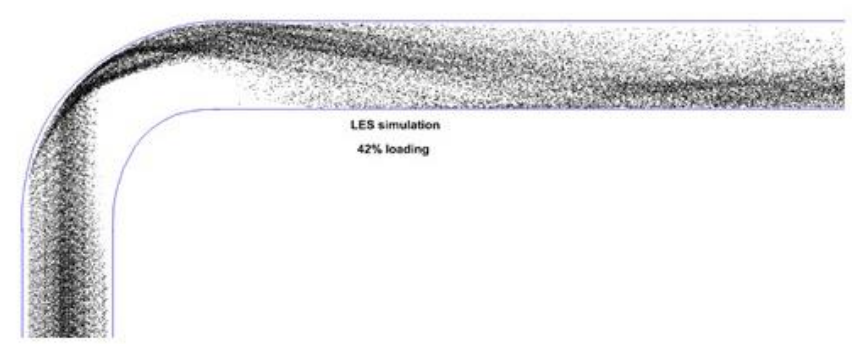

Figure 4.34 Particle visualization from side view for loading of $42 \%$ with $\lambda=1.3$

The cross-sectional particle concentrations for these cases are shown in Figure 4.35 below. It is seen from the figure that, while LES provides better resolution and the ability to predict large eddy structures, the same general trends are shown in both sets of results with respect to the distribution of solids within the pipe cross section. 


RANS

Figure 4.35 Particle concentration contour at different cross sections from RANS (top) and LES (bottom) simulations for $42 \%$ loading condition.

\subsubsection{Summary of Pipe Bend Study}

- The roping phenomenon has been reasonably predicted well by the simulations. The small discrepancy that was observed was that the peak concentration was slightly below compared to experiments. The change in the coefficient of restitution led to much more accurate prediction of concentration profiles and roping behavior.

- A new technique of particle data measurement has been discussed and results were compared to simulations. This study discusses a non-intrusive optical method of studying the roping characteristics without any interference to the flow field by means of an external probe. 
- The rope thickens as the loading is increased. This can be evidently seen from the flow visualizations.

- In RANS simulations, the particles move more towards the lower wall and get deposited. They do not get re-entrained in the flow. This needs to be rectified by making necessary changes to the model in order to re-entrain particles back into the flow.

\subsection{Clustering in a Fluid Bed Riser}

In most of the past numerical studies, several researchers discuss the rope characteristics and effect of several factors on the rope. Evidently, it has been taken for granted that a rope forms in the pipe bend due to inertia and centrifugal effects. Since the afore-mentioned flows are all dilute flows and the gas affects particle behavior, there has to be definitely a role of the gas in rope formation. The eddies and vorticity are likely to have an influence on this. In order to determine the role of dominant flow structures mainly eddies and vorticity in rope formation, numerical simulations of a fluid bed riser have been performed. Here the riser is a rectangular column and hence the centrifugal effects resulting due to shapes like bends are absent.

The riser has a bed of solid particles bed with a certain height and has a freeboard region above the bed. Gas is injected from the bottom of the riser and under certain conditions which makes the entire mixture act as a fluid. There are regular fluid beds risers, circulating fluid bed risers which are used for several applications. These may be dense or dilute in nature. There are several regimes such as particulate fluidization, bubbling, turbulent fluidization and slugging. The bed can have a strong central jet or a uniform flow of air. The solid particle behavior is based on the bed packing, bed height and the velocity of gas. 


\subsubsection{D Simulations using Ansys FLUENT}

The commercial CFD package Ansys FLUENT has been used to perform simulations of a fluid bed riser. The riser has a width of $16 \mathrm{~cm}$ and a height of $80 \mathrm{~cm}$. Eulerian - Eulerian multiphase model has been used for these simulations. The simulation is with uniform velocity and low packing.

\section{Riser with Uniform Inlet}

The bed has a height of $50 \mathrm{~cm}$ with a packing of 0.1 . This riser has a uniform inlet with gas velocity of $1.8 \mathrm{~m} / \mathrm{s}$ or $180 \mathrm{~cm} / \mathrm{s}$. Figure $4.36 \mathrm{a}$ below shows the contour plots of instantaneous solid fraction for different grids at same instant of time. Particle clusters can be clearly seen. These clusters keep oscillating to and from both the walls. Figure $4.36 \mathrm{~b}$ shows the contour plots of time-averaged solids fraction for different grids. The plots resemble each other closely with the averaged concentration being high at the walls. 

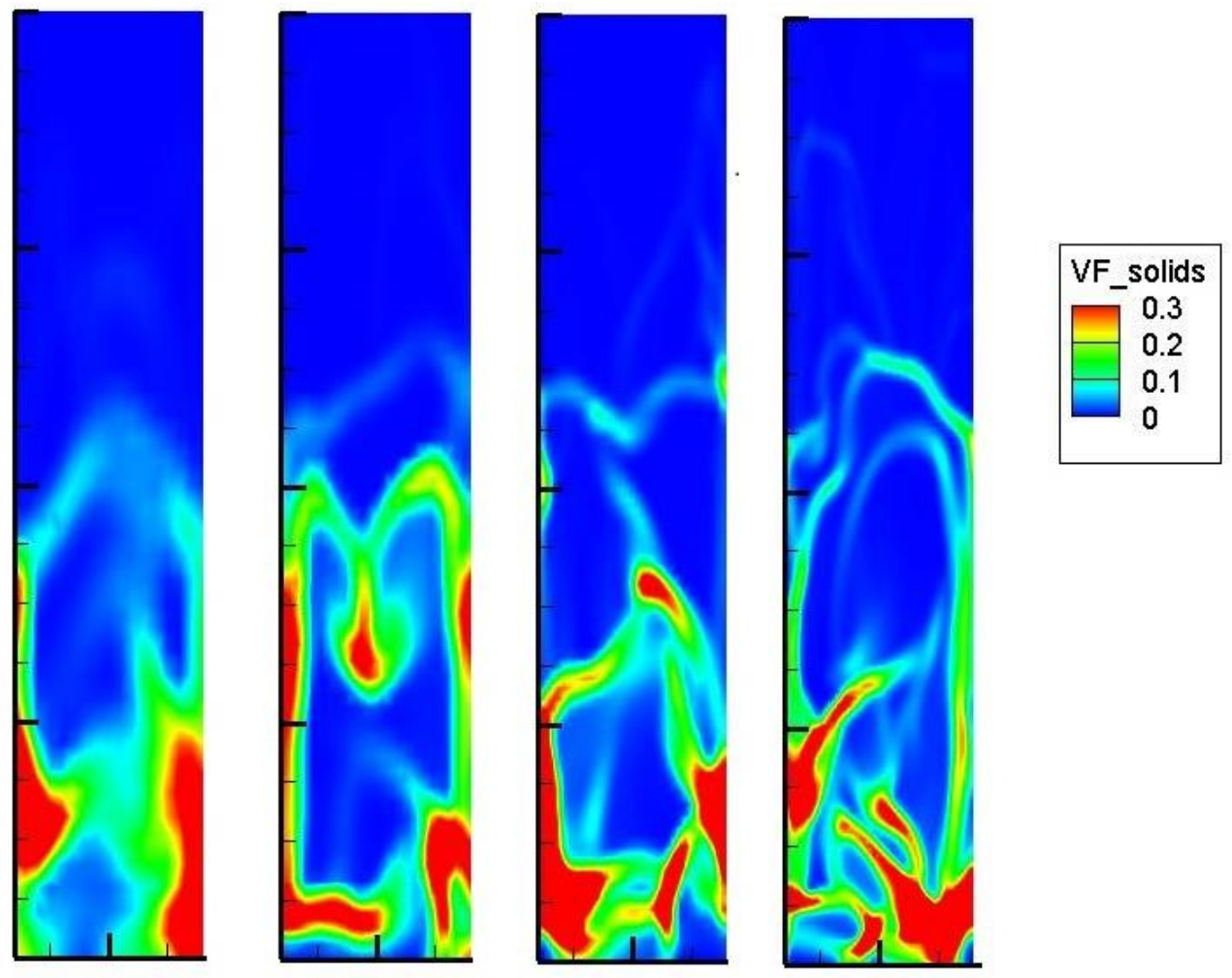

a) Instantaneous solids fraction contours for various grids 


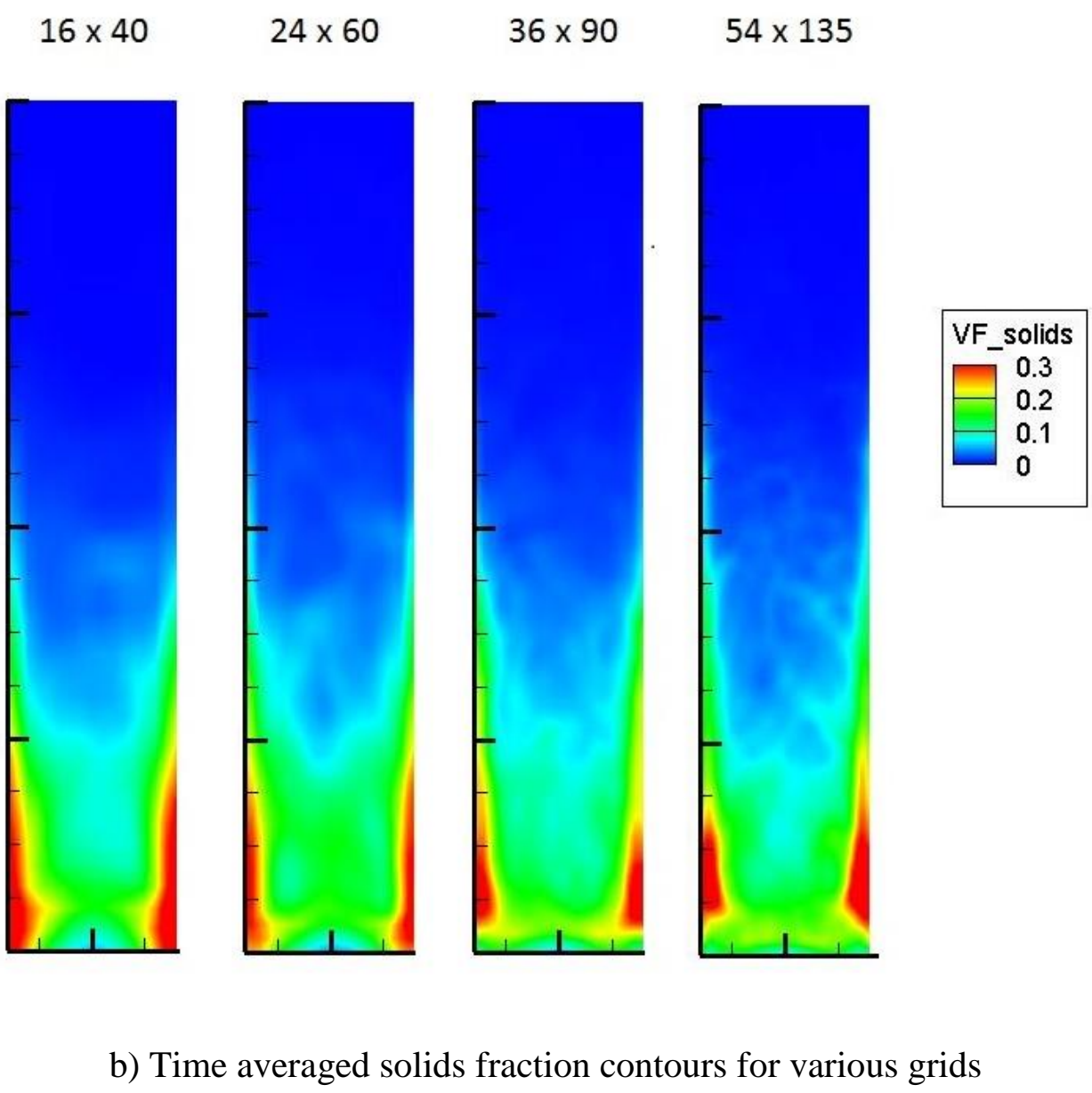

Figure 4.36 Contour plots of solids fraction for several grids with uniform inlet case with FLUENT

\subsubsection{D Simulations using MFIX}

MFIX - Multiphase Flow with Interphase Exchanges is a hydrodynamic model generally used to model dense and dilute solid-liquid or solid-gas flows. These two-fluid hydrodynamic models treat fluid and solids as continuum. The riser with a width of $16 \mathrm{~cm}$ and a height of $120 \mathrm{~cm}$ was simulated to help achieve stable runs. The grids have been consistently refined in the $\mathrm{X}$ and $\mathrm{Y}$ directions for the increased length of the domain, maintaining the same cell sizes. The simulation is with uniform velocity and low packing. 


\section{Riser with Uniform Inlet}

The bed has a height of $50 \mathrm{~cm}$ with a packing of 0.1 . This riser has a uniform inlet with gas velocity of $1.8 \mathrm{~m} / \mathrm{s}$ or $180 \mathrm{~cm} / \mathrm{s}$. Figure 4.37 a below shows the contour plots of instantaneous solid fraction for different grids at same instant of time. Particle clusters can be clearly seen. These clusters keep oscillating to and from both the walls. Figure $4.37 \mathrm{~b}$ shows the contour plots of time-averaged solids fraction for different grids. The plots differ from each other a lot. The clusters are thin and located on the wall as seen from the time averaged plots.

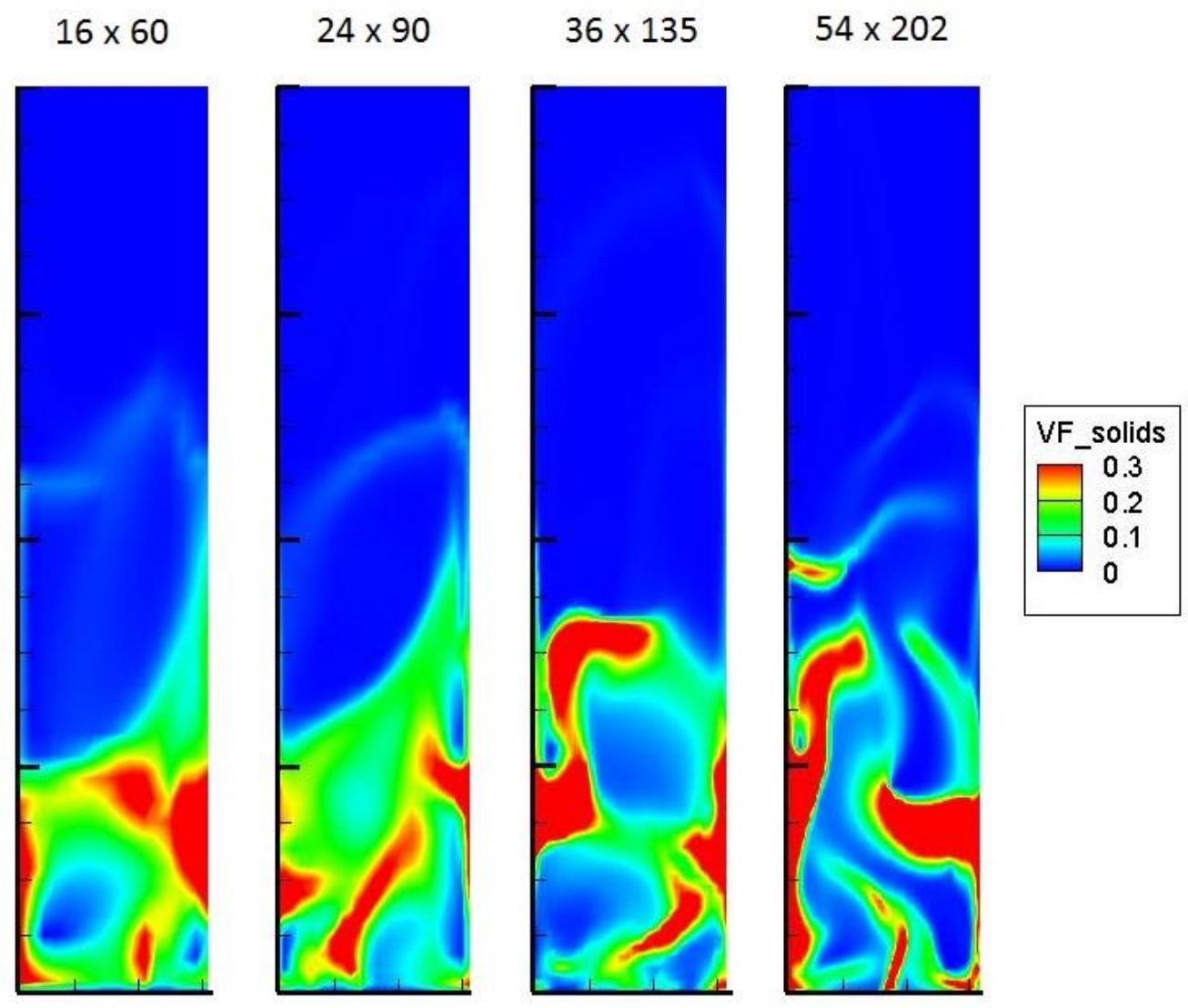

a) Instantaneous solids fraction contours for various grids 


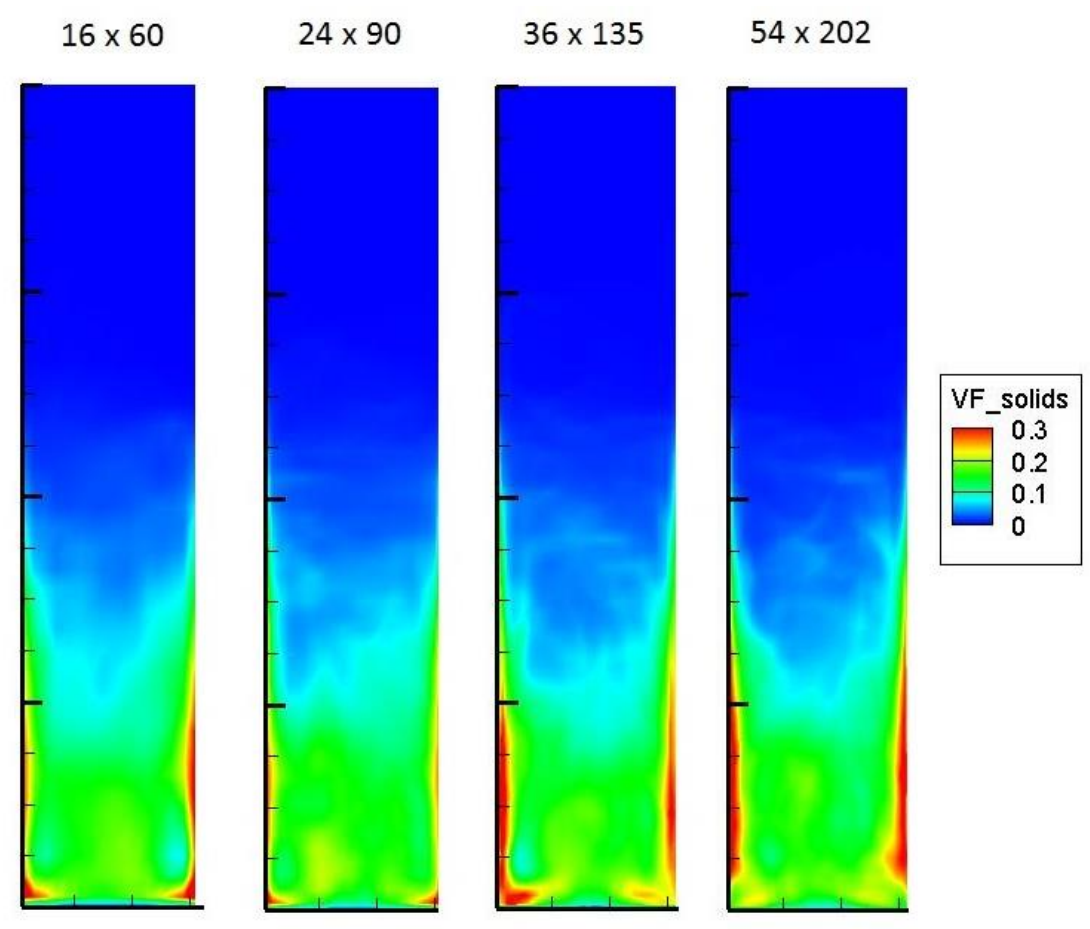

b) Time averaged solids fraction contours for various grids

Figure 4.37 Contour plots of solids fraction for several grids with uniform inlet case with MFIX

\subsubsection{Comparison of FLUENT and MFIX Results}

Fluid bed riser has been simulated using both MFIX and FLUENT. The Eulerian-Eulerian Multiphase models have been used in both of these. Figure 4.38 shows and compares the cluster formation in MFIX and FLUENT according to the cluster identification and characterization criteria [43]. Both these show that the clusters are surrounded by vortices of opposite directions and at the location (central region) of cluster, vorticity is low. Though it is a known fact that 2D simulations may lead to a lot of unphysical particle accumulation, the main objective here was to show the vorticity behavior with clusters. 

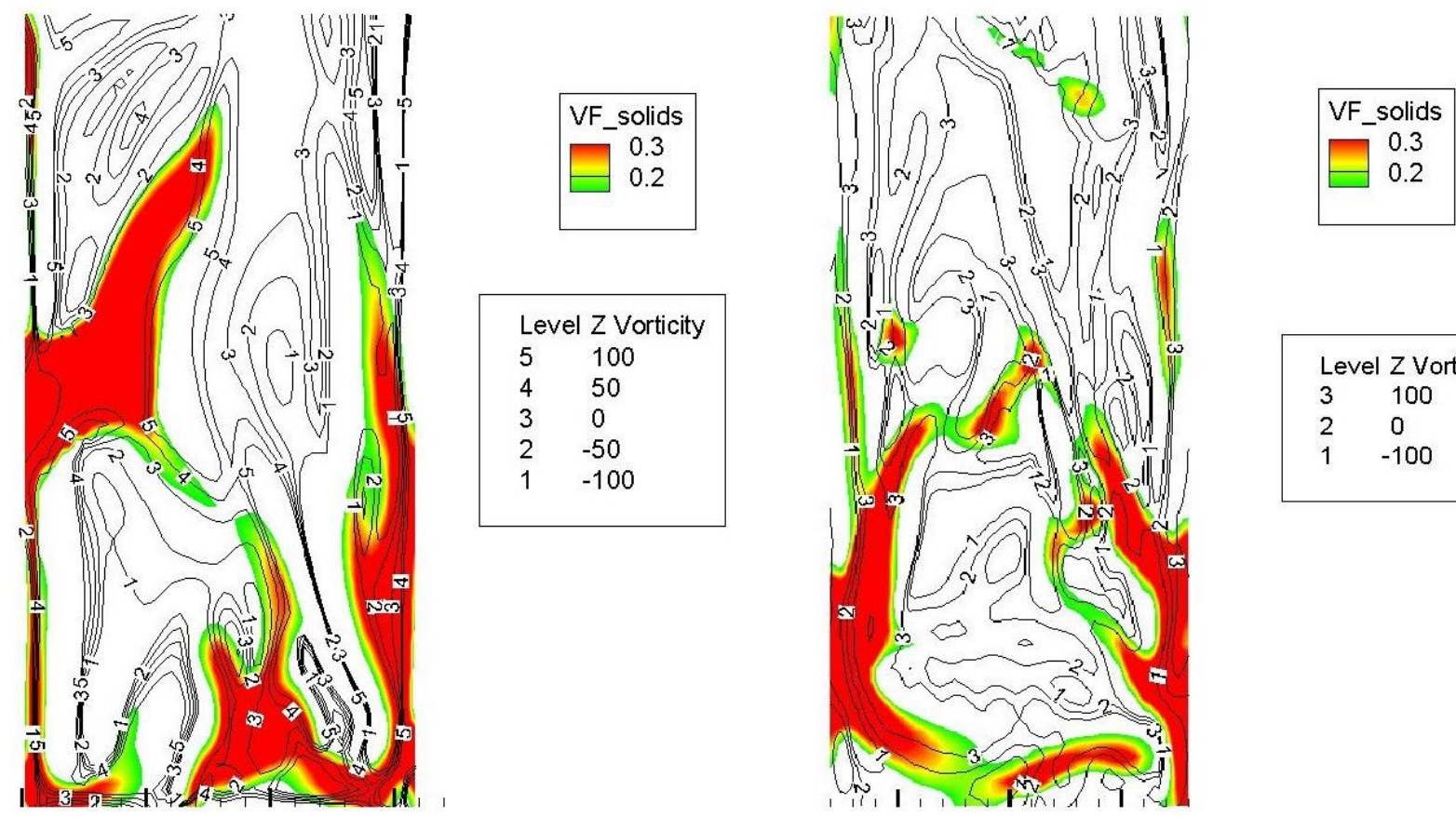

Figure 4.38 MFIX and FLUENT showing particle clusters

\subsubsection{Summary of Fluid Bed Riser Study}

From the above simulations from FLUENT and MFIX, the clustering effect can be clearly seen in 2D fluid bed risers. The analysis of clustering with vorticity suggests a dominant role of vorticity in cluster or rope formation. The results from $2 \mathrm{D}$ simulations indicate that the high concentration regions and vorticity are correlated strongly but there was no indication of what caused what. Both MFIX and FLUENT successfully predicted particle clustering for dilute cases with uniform inlets. MFIX results showed error propagation (Appendix A) in grid independent study which creeps in due to the grid and also on account of time averaging. 


\section{Chapter 5 Vorticity and Turbulence Kinetic Energy Effect}

In this study, the main aim is to find the relation between the gas vorticity and the high particle concentration regions; in particular which influences the other. Numerical simulations have been performed on a vertical 3D pipe by creating a vortex field. Very Large eddy simulations (VLES) with lagrangian Discrete Phase model (DPM) have been performed using the commerical CFD software Ansys FLUENT. The effects of several factors such as particle size and injection velocity on the particle clustering have also been studied. The correlations among turbulent kinetic energy, vorticity, and particle clustering and roping are analysed. Additional simulations were performed using the MFIX software [44] which is more suitable for dense gas-solid flows. The MFIX results are compared to those obtained from Ansys FLUENT.

\subsection{Turbulence Modeling}

Large eddy simulations (LES) have been performed on coarser grids compared to the typical LES grids. To distinguish the present results from regular LES, we shall refer to them as VLES from here on. In LES method, the large eddies are resolved and small scales are modeled. Filtering operation with help of a spatial filter separates these scales. The governing equations for the LES are the filtered Navier Stokes equations. The effects of the smaller eddies are incorporated by means of a sub-grid scale model. LES is therefore computationally less expensive compared to a Direct Numerical Simulation (DES) where all scales are resolved. Sub-grid scale model used in this study is the Smagorinsky-Lilly model with a Smagorinsky constant, $C_{S}$, value of 0.1 . Since we use relatively coarse meshes, we expect to capture only relatively large scales / structures in the order of say $1 / 10^{\text {th }}$ of the scales imposed by the geometry of the problem. 


\section{Vortex Generation}

An inlet vortex method with a random nature has been used to create a vortex field. Perturbations are added on the mean velocity profile by means of a fluctuating vorticity field. This method is based on Lagrangian form of 2D vorticity evolution equation and the Biot-Savart law. Vorticity carried by a randomly convected vortex point is given by the circulation, $\Gamma$,

$$
\Gamma(x, y)=4 \sqrt{\frac{\pi A k(x, y)}{3 N[2 \ln (3)-3 \ln (2)]}}
$$

where $\mathrm{N}$ is number of vortex points, $\mathrm{A}$ is the inlet area and $k$ is the turbulent kinetic energy.

In FLUENT, a linear kinematic model is used for the stream wise fluctuations [45]. This model mimics the influence of a $2 \mathrm{D}$ vortex in the mean stream wise velocity field. Considering mean stream wise velocity $\mathrm{U}$ as a scalar, the fluctuation $u$ ' arising from the transport of $\mathrm{U}$ by planar fluctuating velocity field $v^{\prime}$ is given by:

$$
u^{\prime}=-\vec{v}^{\prime} \cdot \vec{n}
$$

Here $\vec{n}$ is a unit vector aligned along mean velocity gradient, $\nabla U$.

\subsection{Model Setup}

\section{Mesh}

A 3D vertical fluid bed riser has been modeled in this study. The diameter of the pipe is $16 \mathrm{~cm}$ and the height of the pipe is $80 \mathrm{~cm}$. The inlet is a velocity inlet and the outlet is a pressure outlet. The walls of the pipe have no slip boundary condition. In this study, Very Large Eddy Simulations (VLES) have been performed. The grid for VLES simulations is relatively coarse compared to the 
LES simulations. The VLES grid in this study has 184320 cells. The grid for VLES is shown below in the Figure 5.1.

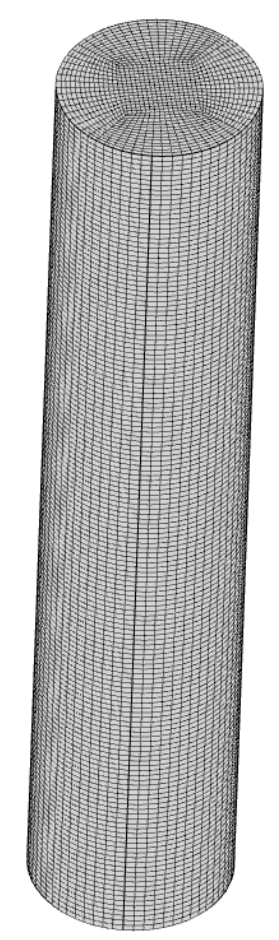

Figure 5.1 VLES Grid

\section{Operating Conditions and Simulation parameters}

The material used for these simulations is characterized with a density of $2.71 \mathrm{~g} / \mathrm{cc}$. The solids-gas loading based on mass flow rates is $10 \%$. The operating conditions used for these simulations are given below in Table 5.1 . 
Table 5.1 Operating Conditions for all simulations in this study

\begin{tabular}{|c|c|c|c|}
\hline Case No. & Inlet Gas & Inlet Solids & Particle \\
& Velocity & Velocity & Diameter \\
\hline 1 & 1.8 & 0 & 40 microns \\
\hline 2 & 1.8 & 0 & 200 microns \\
\hline 3 & 1.8 & 0.6 & 40 microns \\
\hline
\end{tabular}

The Discrete-Phase Model of Ansys FLUENT has been used with unsteady particle tracking for these simulations. Shape factor of 0.755 has been used for the particles. The wall coefficient of restitution is 0.9. The Pressure-Velocity Coupling is SIMPLE, spatial discretization for pressure is Standard and for momentum Bounded Central Differencing has been used. The transient formulation is second order implicit. The time step size is 0.0001 . First the simulations were run till the gas flow field has been established and only then particles were introduced.

\subsection{Results and Discussion}

Several simulations have been performed to study the relation between vorticity and particle concentration. Contour plots with particle concentration overlapped by vorticity (perpendicular to the plane of view) at various cross-sections in height are shown in Figure 5.2a and Figure 5.2b after nearly five flow through times. The concentration and vorticity have been normalized. All the concentration values above 0.2 fall into above 0 range and those lesser fall below the 0 range. All the normalized concentration values nearing 1 or above represent the peak concentration cluster regions. Vorticity values have been normalized by 5 to clearly indicate low vorticity regions with opposite orientations. Circles have been drawn marking areas of high concentration which indicate clusters surrounded by clockwise and anti-clockwise vortices. 

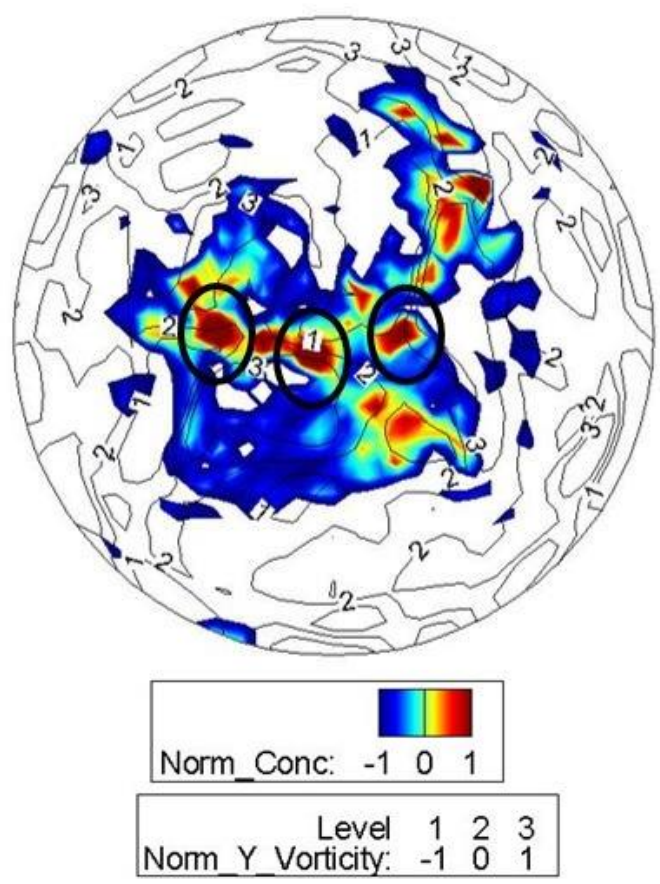

a)

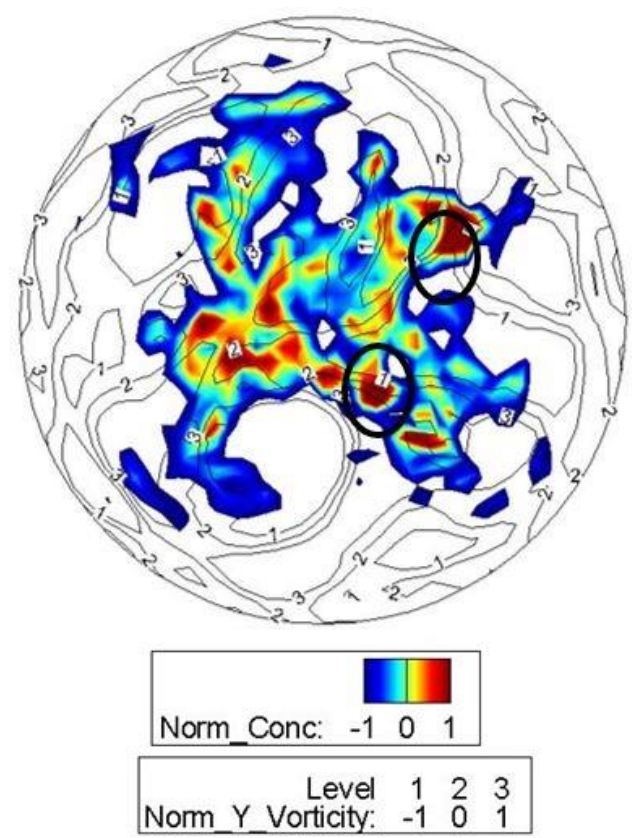

b)

Figure 5.2 Cross-sections at height a) $40 \mathrm{~cm}$ b) $50 \mathrm{~cm}$ showing normalized particle concentration $\mathbf{C n o r m}=\frac{\boldsymbol{C}-\mathbf{0 . 2}}{\mathbf{0 . 0 5}}$ overlapped by normalized Y-vorticity (w/s) in direction of flow 
It can be clearly seen from Figure 5.2 that the clusters are surrounded by vortices of opposite orientation and at the location of the cluster, the vorticity is nearly zero or very low.

Figure 5.3a shows the enlarged pictured of a cluster at a cross-section at a height of $50 \mathrm{~cm}$ from case 1 . Figure $5.3 \mathrm{~b}$ shows the plots of normalized concentration and normalized vorticity along the normalized length of the line. We can clearly see that where the peak concentration occurs, the vorticity is zero or very low. It can also be seen that the peak concentration has vortices of opposite direction on either side. At low concentration regions, the vorticity value is higher.

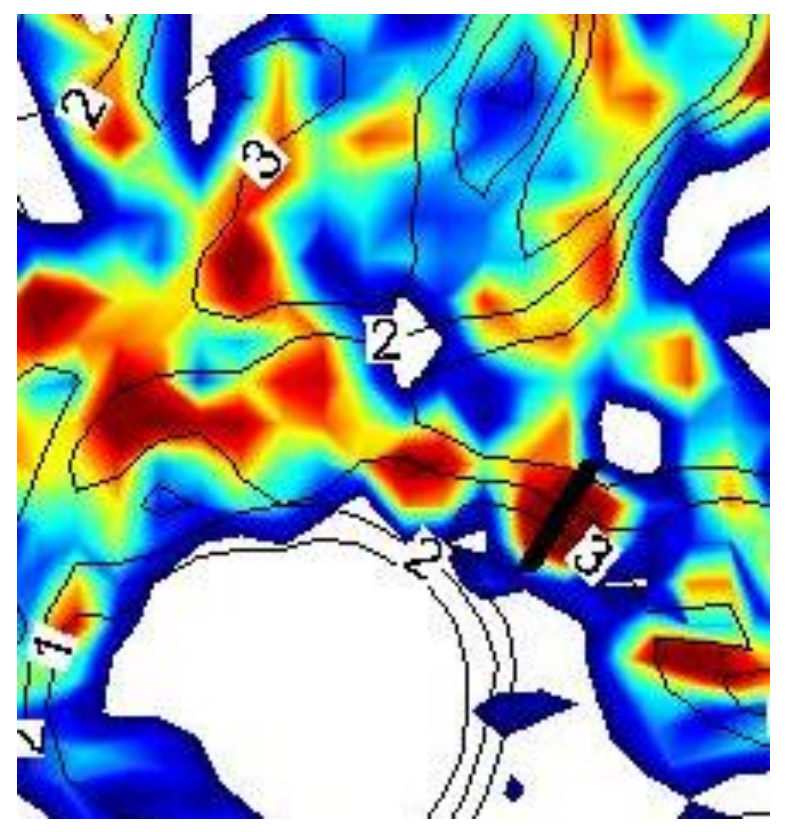

a) Line on a cluster 


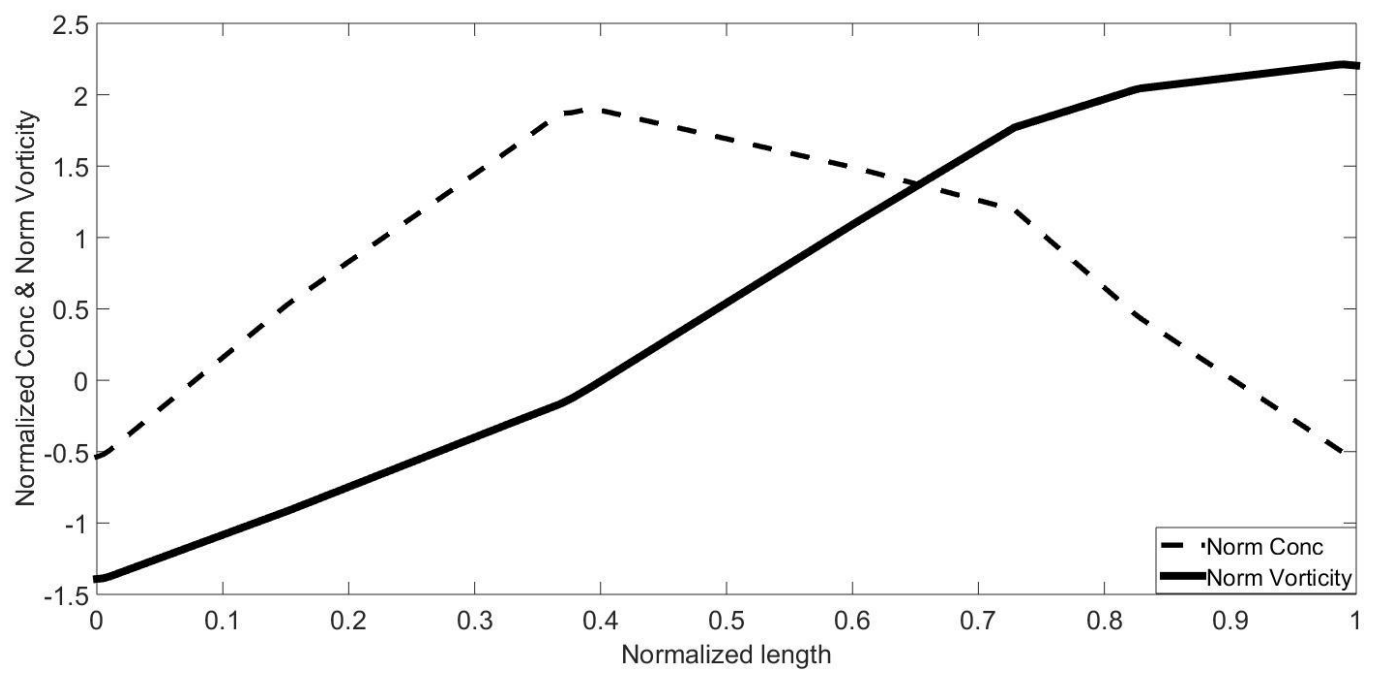

b) Normalized Concentration and Normalized Y-Vorticity along the normalized length of the line

Figure 5.3 Detailed analysis of a cluster location

Figure 5.4 shows the cluster concentration surrounded by $\mathrm{Z}$ vorticity along a vertical plane. It can be observed that the same phenomenon as discussed above occurs.
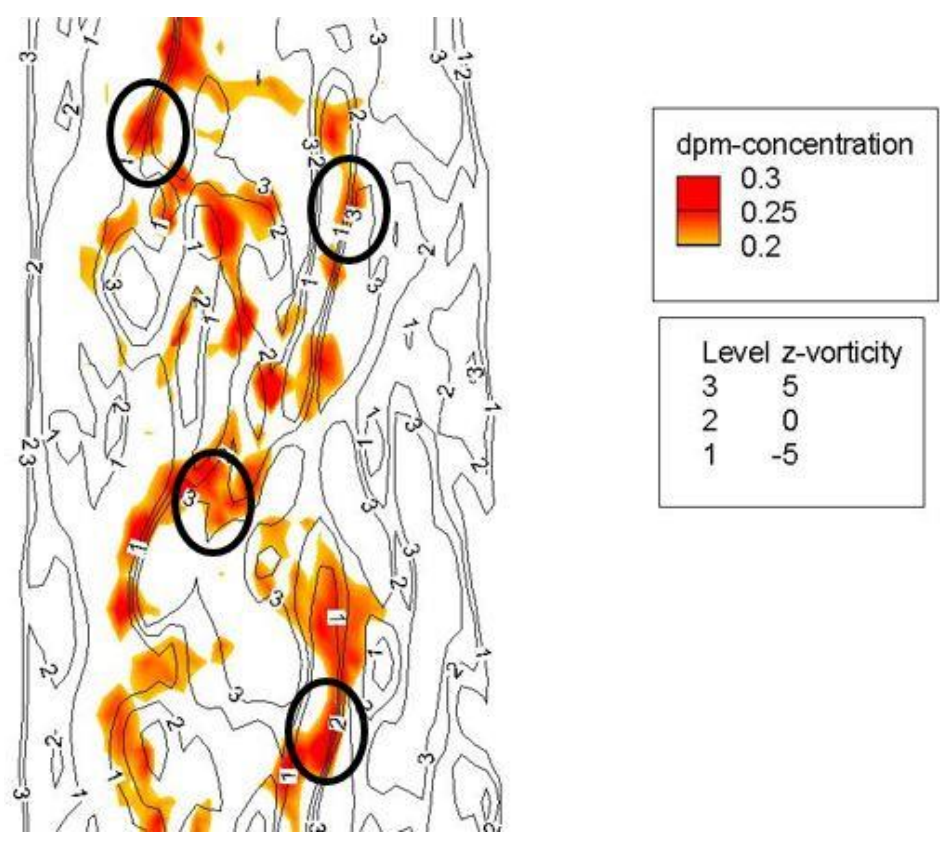

Figure 5.4 Vertical cross-section with actual concentration with overlapped Z-vorticity 
From the above figures, it is observed that clusters are surrounded by vortices of opposite orientation on all sides. Figure 5.5 shows clearly the mechanism of cluster or rope formation by a roller effect. Here concentration is overlapped by vectors. Vortices of opposite orientation drive particles into the space between them and sandwich the particles to form a region of high concentration forming a cluster or a rope. This may be referred to as the "roller effect" of vortices in formation of clusters.

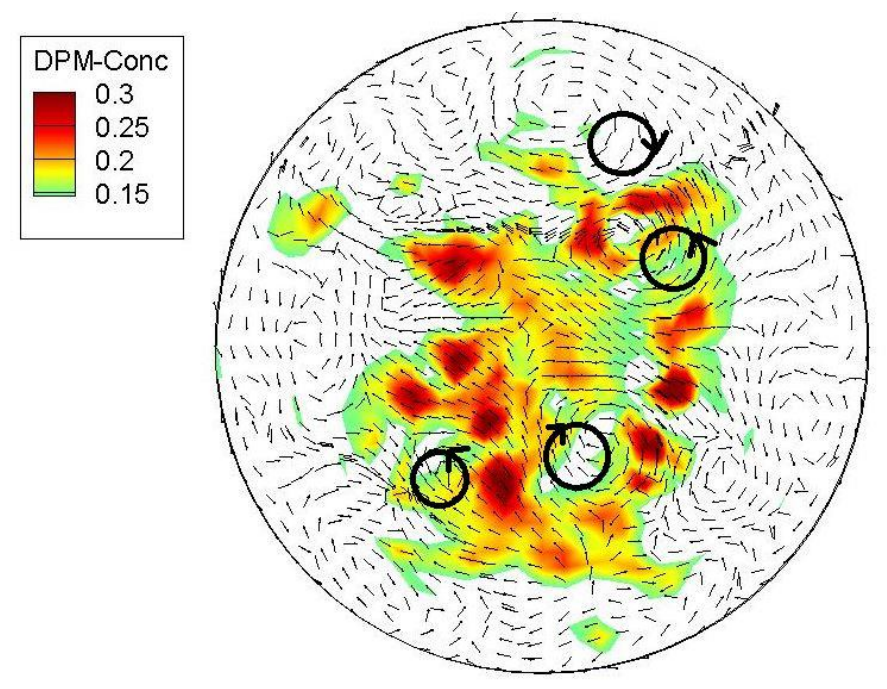

Figure 5.5 Roller effect of vortices in cluster or rope formation

Figure 5.6 shows the particle clustering phenomenon in cases 1,2 and 3 respectively. On comparison of three cases, it can be seen that in the case where 40 micron particles are injected with 0 velocity, clusters appear to be small islands of particles with thin branches. In case 2 where 200 micron particles are injected with 0 velocity, the concentration is atleast three times compared to case 1 and 3. Very thick clusters or ropes are formed with large particles. In case 3, where 40 micron particles are injected with a velocity $0.6 \mathrm{~m} / \mathrm{s}$, the cluster concentration is similar to case 1 but the branches are wide and spread out in the cross-section. 

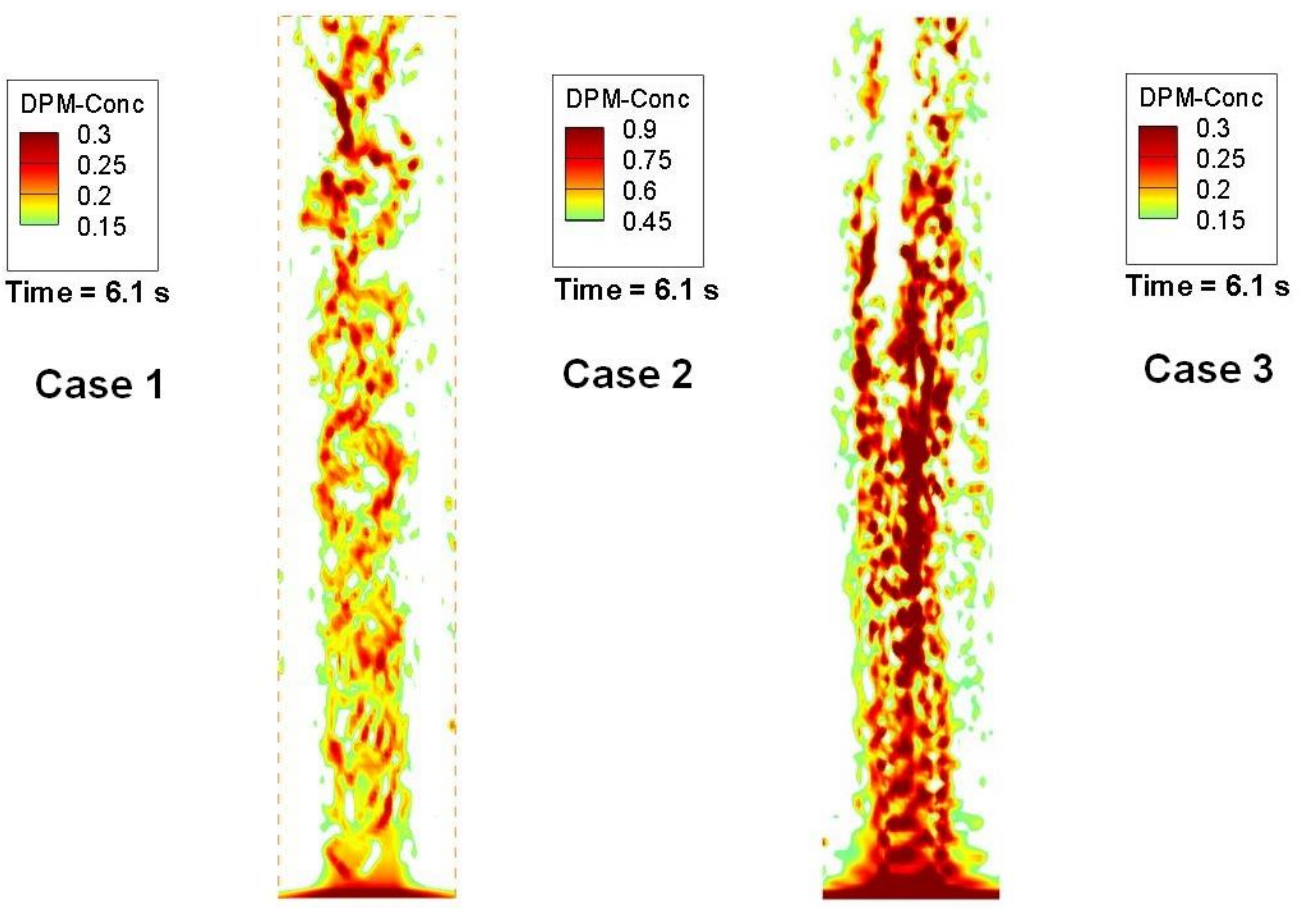

\section{Case 2}
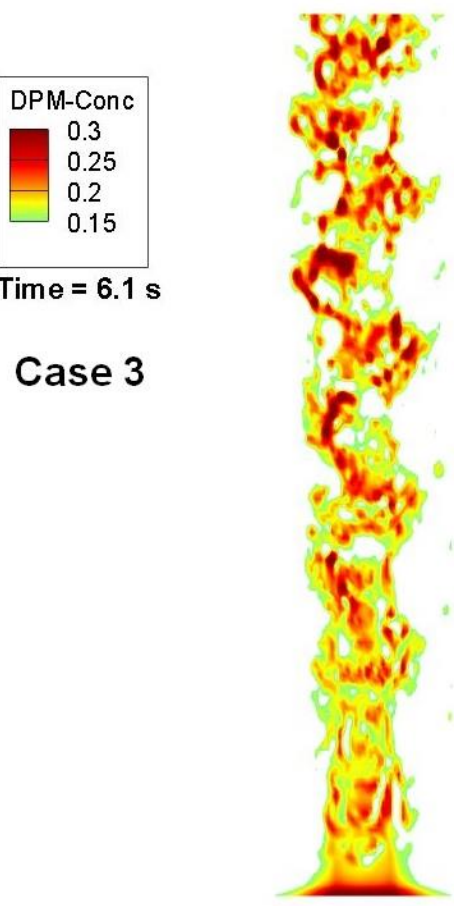

Figure 5.6 Comparison of clusters in cases 1, 2 and 3 respectively shown here are instantaneous

In order to analyze if the particles cause vorticity or the vorticity causes clustering, three cross sections have been taken along the height in Y direction at heights $20 \mathrm{~cm}, 40 \mathrm{~cm}$ and $60 \mathrm{~cm}$. Area weighted average plots of vorticity magnitude have been plotted over two flow through times for the gas flow and also for the gas flow with particles. These plots are shown in the Figure 5.7. The mean area weighted average vorticity magnitude for each cross-section over time has also been shown in the figure. It can be clearly seen in the Y-cross sections that the mean time averaged area weighted vorticity magnitude is nearly the same with and without particles with only a slight increase by a value of $2(1 / \mathrm{s})$ at only after the free board region. 

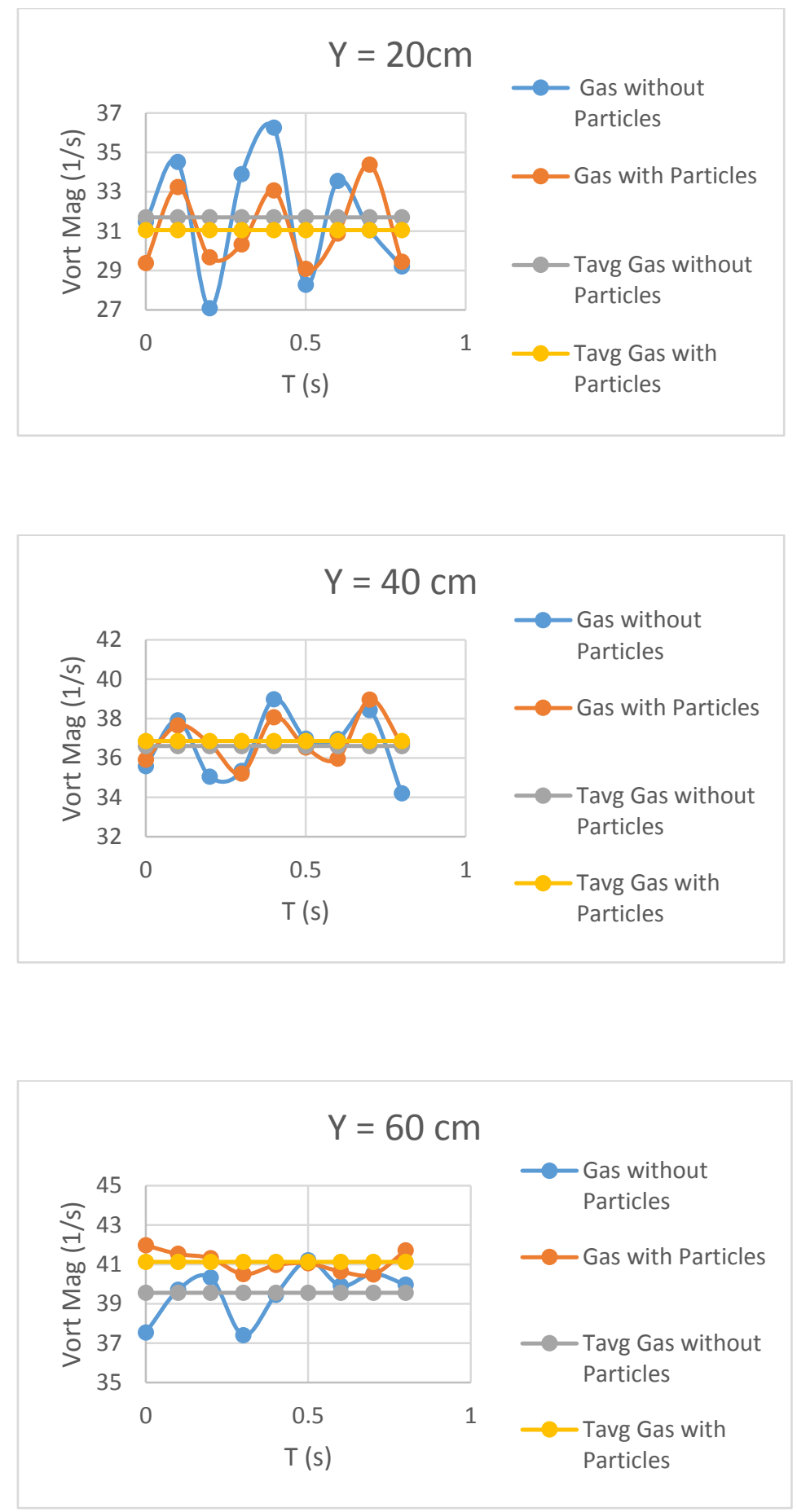

Figure 5.7 Plots of area-weighted average vorticity magnitude along time at various crosssections in height 
At lower Y cross-section, the average is slightly higher of pure gas flow than with the flow with particles. This becomes nearly equal at the central height cross-section and then there is a slight increase in the free board region. From the above plots, it can be observed that there is not a significant increase in the vorticity magnitude.

Figure 5.8 below shows the cluster formation process. The three cross-sections in height are shown with contours of particle concentration overlapped with vorticity magnitude in the first row and overlapped with Y-vorticity (flow direction) in the second row.

Since this is a dilute flow and the flow influences particles a lot, this decrease in vorticity from lower cross-section to higher cross-section can be attributed to the effect of vortices in bringing the particles together to form clusters. The transformation from islands of small particle clusters to branches of clusters occurs in this stage. This is clearly evident in the left column top and bottom contours of Figure 5.8. Nearing the central height of the riser the islands of particles form branches. The mean vorticity magnitude being nearly equal can be interpreted as the balance in the vortices forming branches and the clusters shedding small wakes of vortices. This is evident from the center column top and bottom figures in Figure 5.8. The small increase in vorticity from central region to above the freeboard region can be interpreted as small wake vortices released by the formed high concentration larger cluster. These wakes can further cause particles to add up to the cluster. The formation of a higher concentrated cluster in shown in the last column top and bottom figures of Figure 5.8. All the three cluster regions have low vorticity and are surrounded by vortices of clockwise and counter clockwise direction. 

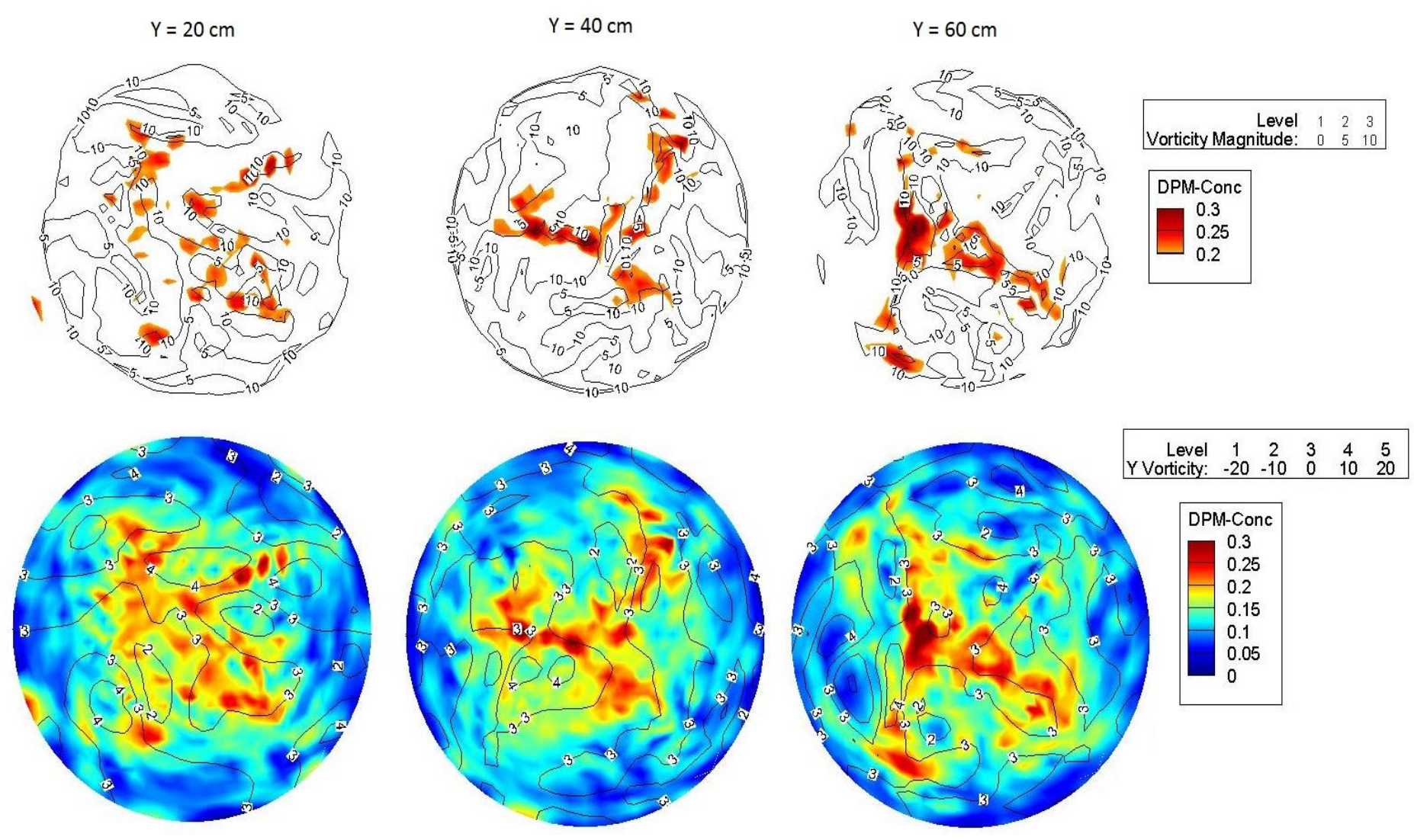

Figure 5.8 Contours of concentration overlapped by vorticity magnitude (top row) and Yvorticity (bottom row)

Mathematically by observing the marginal vorticity magnitude increase with particles, it can be said that the only weak vortices may be shed. With the cluster being surrounded by vortices of higher magnitude and opposite directions, and Y-vorticity being very low or zero, the magnitude can only be low with weak vortices. So this proves that, the presence of cluster in between two opposite direction higher vortices is due to its formation by the vortex roller mechanism which is indeed the cause and not the effect.

Summarizing this effect, the flow first creates small islands of particle clusters which then grow to branches and eventually form high concentration clusters, all of these occurring by the vortex 
roller effect. These large clusters produce weak vortices in wakes which aid in cluster growth too. The vortices surrounding the cluster deform these clusters to stretch into rope like structures.

\section{Riser simulation in MFIX}

A 2D Fluid bed riser simulation with the same geometry has been performed using MFIX code developed by NETL. The fluid bed has a bed height of $50 \mathrm{~cm}$ with a solid packing of 0.1.The inlet gas velocity is $1.8 \mathrm{~m} / \mathrm{s}$. Figure 5.9 shows the solids volume fraction overlapped with vorticity. This has been performed with the two fluid model, eulerian-eulerian approach.

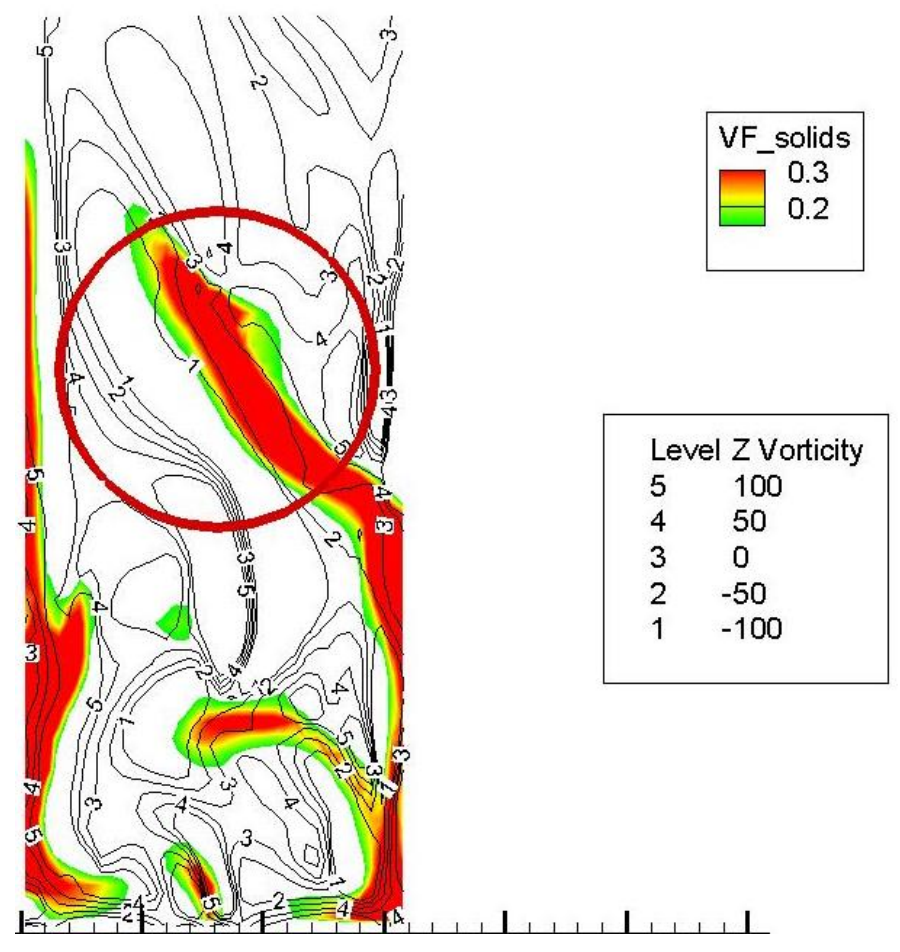

Figure 5.9 2D Fluid bed simulation with MFIX: Concentration vs Vorticity

It can be clearly seen that roping or clustering characteristics are observed. The clusters or rope is surrounded by vortices rotating in opposite direction while at the high concentration region, the vorticity is zero or very low. 
Figure 5.10 shows the contour plots of concentration overlapped by turbulent kinetic energy (T.K.E). This plot clearly shows that at high concentration regions, the T.K.E is low and at low concentration regions, T.K.E is relatively higher.

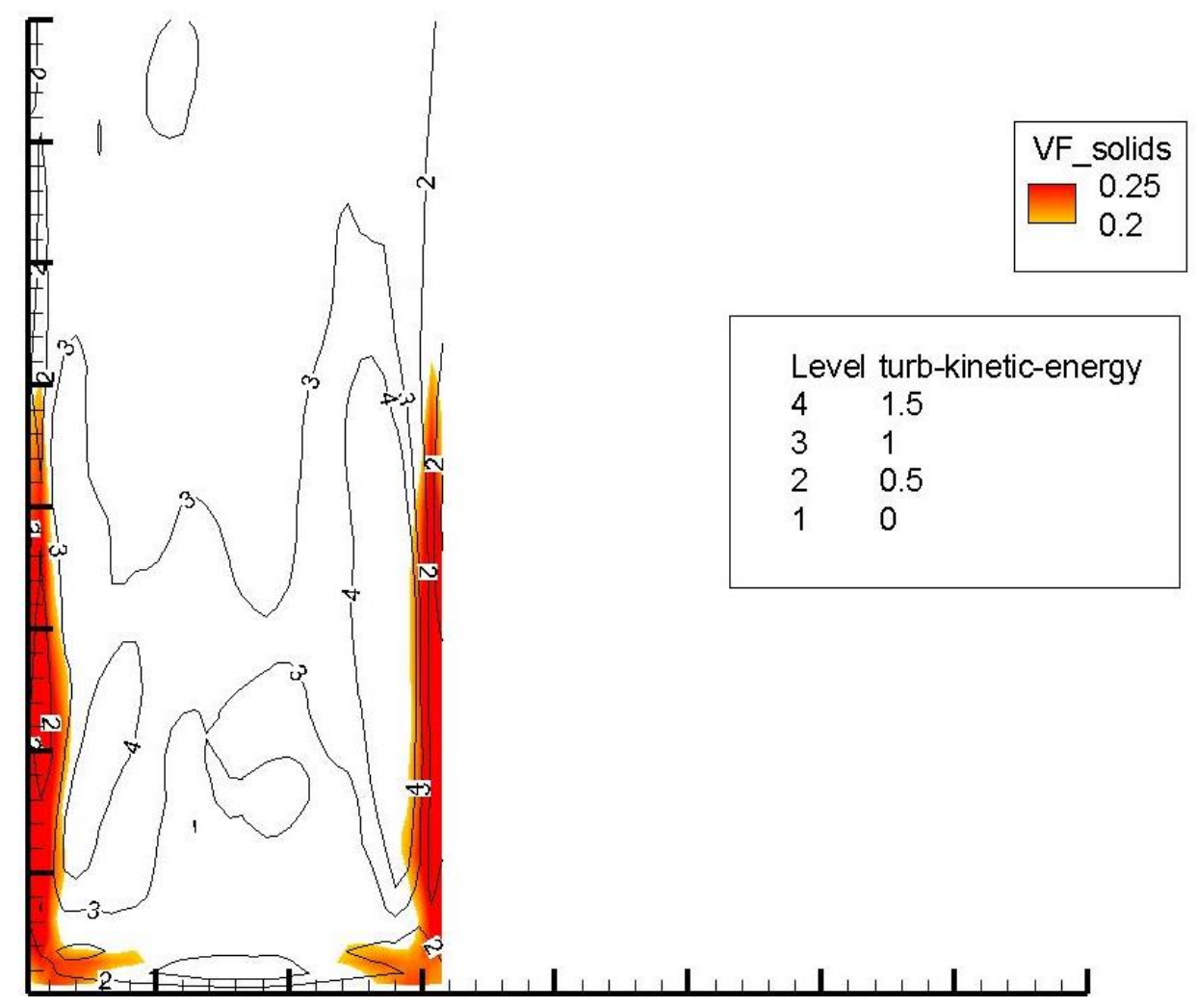

Figure 5.10 2D Fluid bed simulation with MFIX: Concentration vs T.K.E

\section{Pipe bend analysis}

Similar mapping analysis was performed on the earlier simulations for gas-solid flows at a loading of 42 percent in a vertical to horizontal 90 degree elbow. The concentration along a cross-section was plotted against vorticity, vorticity with T.K.E and T.K.E with concentration in Figure 5.11a, Figure 5.11b and Figure 5.11c respectively. It can be seen from Figure 5.11a, at location of high concentration or the rope, the vorticity is zero and the rope is surrounded by clockwise and counter- 
clockwise vortices. The vortex roller effect involved in rope formation is clearly seen in Figure 5.11b. Figure 5.11c shows that at high concentration region, T.K.E is low. While the larger vortices cause particles to cluster together, the weak vortices give the random shape to the clusters.

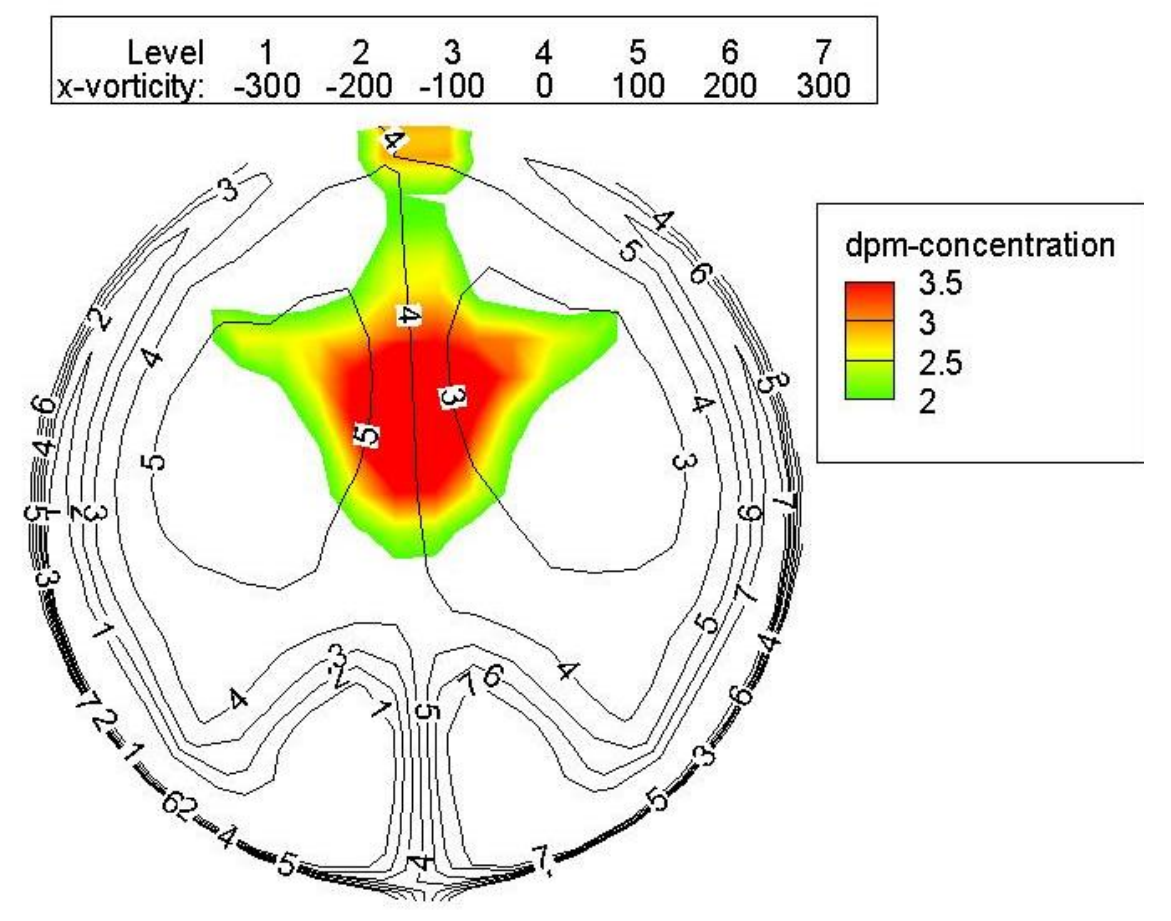

a) Concentration Vs Vorticity 


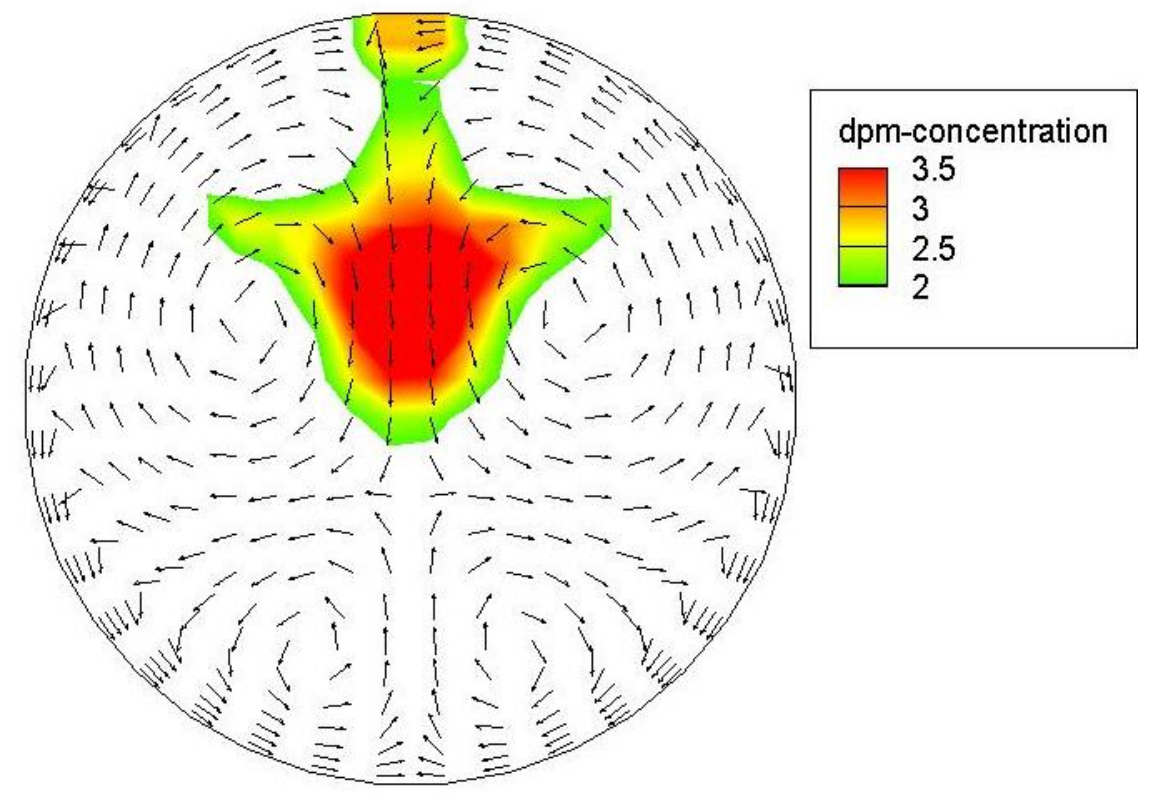

b) Vortex roller effect in rope formation

\begin{tabular}{rccccccccc|}
\hline Level & 1 & 2 & 3 & 4 & 5 & 6 & 7 & 8 & 9 \\
turb-kinetic-energy: & 0 & 0.2 & 0.4 & 0.45 & 0.5 & 0.6 & 0.8 & 1 & 1.2 \\
\hline
\end{tabular}

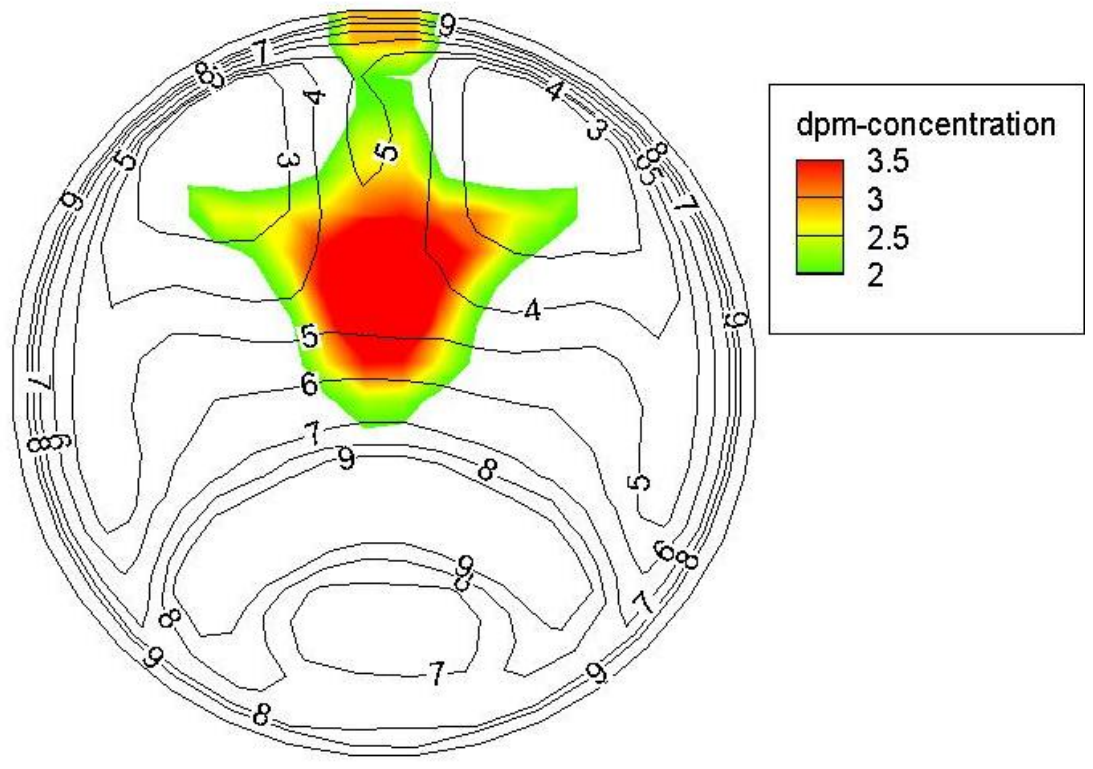

c) T.K.E Vs Concentration

Figure 5.11 Contours of variables at location downstream of pipe bend 


\subsection{Summary of the Vorticity and T.K.E Study}

Gas-solid flows in a vertical riser have been simulated for different flow conditions. It can be clearly observed from all the above simulations that rope or clusters of particles have formed in response to vorticity dynamics. This effect is basically a "roller effect" whereby vortices rotating in opposite directions gather/collect the particles in between them to form rope like structure. Multiple small clusters from different directions can come together to form a rope like structure with high particle concentration. In pipe bends, it is commonly stated that rope forms due to centrifugal effects of the bend. From the above simulations, it can be stated that it is not only the effect of geometry but also the flow by means of vortices plays a significant role in rope formation. Therefore rope formation can be redefined as clustering and deformation of particle structures between vortices rotating in opposite directions having similar magnitude. This is also a result of effect of bend geometry. It is further observed that at regions of high particles concentration T.K.E is low and at regions of low particle concentration T.K.E is high. The Figure 5.12 below summarizes the development of high particle concentration clusters or ropes. 

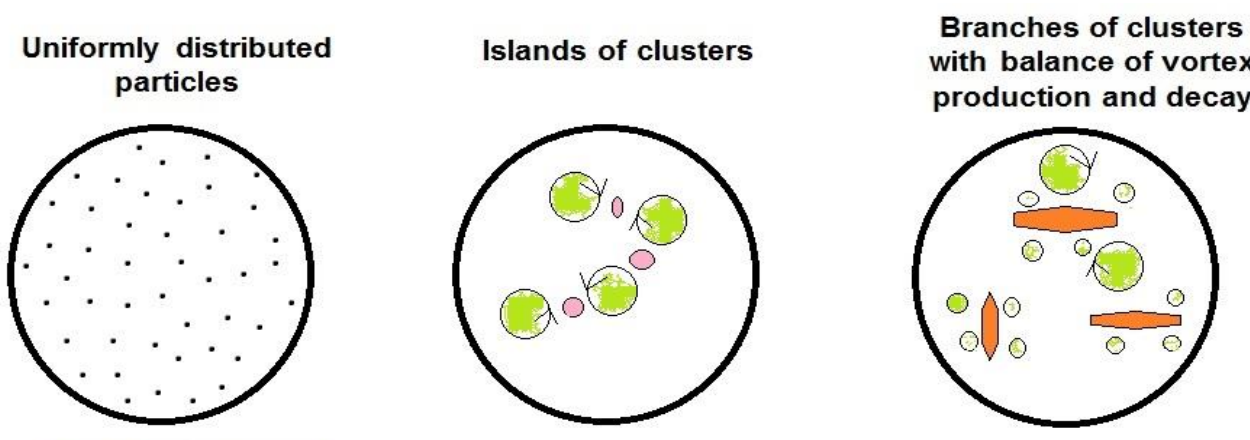

High Concentration Clusters with weak vortex production
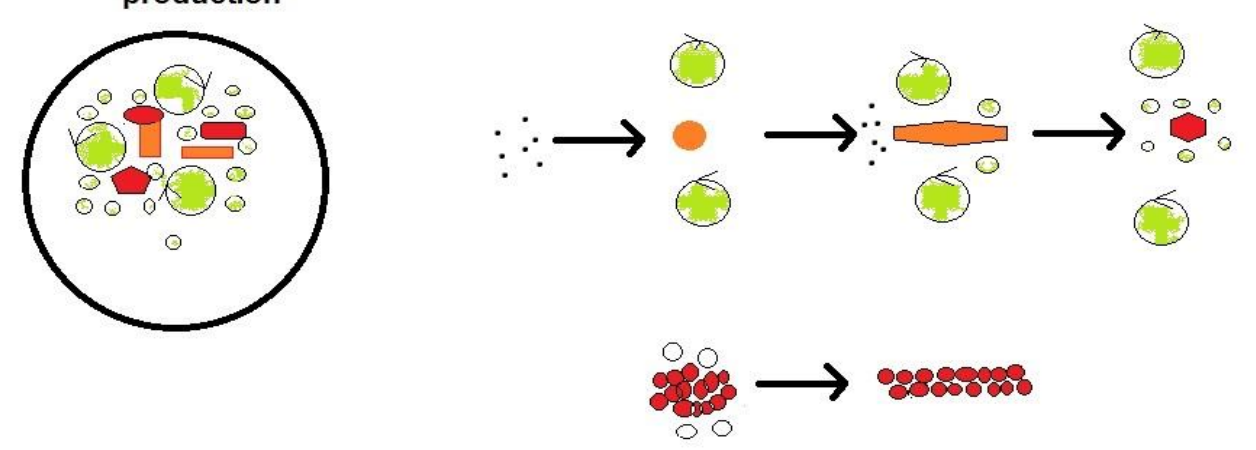

Stretching of cluster to rope

Figure 5.12 Map summarizing formation of ropes 


\section{Chapter 6 Conclusions}

Gas-solid flow simulations with a purpose of understanding the physics behind roping phenomenon have been performed for pipe bends and fluid bed risers. The particle clustering behavior in both these geometries has been studied using different multiphase flow models. The results of simulations in pipe bends with Ansys FLUENT have been supported by experiments and simulations of fluid bed risers with simulations using MFIX code.

- Numerical simulations of Cyclone classifiers showed discrepancies in experimental and simulation results. Several turbulence models, injection conditions and several other factors have been studied which however failed to improve the predictions. The reason behind these problems was found to be models not accounting for the roping phenomenon.

- Experiments have been performed to capture roping and analysis of the flow behavior was performed with the help of high speed imaging. The experiments and simulations with correct coefficient of restitution captured roping very well and results matched well with each other.

- Coefficient of restitution was found to be a major factor which influences prediction of roping in simulations especially ratio of tangential component to normal component.

- Multiphase flow simulations were performed on a fluid bed riser to capture and study the clustering phenomenon. From this study, it was found that particle clusters are formed due to action of opposite rotating vortices which bring particles together to form a rope- known as the "vortex roller effect". This roping behavior was predicted in risers with Ansys FLUENT and also by MFIX software.

- Particle rope can be fundamentally defined as a "region of high particle concentration brought about as a result of clustering of particles between vortices of opposite 
orientation" and can also be formed due to the centrifugal effects of certain geometries such as bends.

- It was also observed that at high concentration regions such as ropes, the vorticity is very low or nearly zero. Moreover the turbulent kinetic energy value is also low in such regions. In order to predict the cyclone efficiency better, models should incorporate methods that can handle these high concentrations areas better. The models have to take into account the clustering behavior of particles. Turbulence kinetic energy can be utilized as a parameter for model development as high T.K.E regions have low concentration.

- It is proposed that the production and destruction terms which appear in two-equation turbulence models should be made a function of particle concentration even for seemingly dilute flows on the average. This modification to turbulence models is necessary to predict the gas-solid flows more realistically in industrial applications such as cyclone separators with abrupt turns in flow direction causing regions of high particle concentration. 


\section{References}

[1] R. Siddique. Utilization of coal combustion by-products in sustainable construction materials. RECYCL Resources, Conservation \& Recycling 54(12), pp. 1060-1066. 2010.

[2] Blissett, R.S.,Rowson, N.A.,. A review of the multi-component utilization of coal fly ash. JFUE Fuel 97pp. 1-23. 2012.

[3] G. Li, Q. Liu, Z. Liu, Z.C. Zhang, C. Li and W. Wu,. Production of calcium carbide from fine biochars. Angewandte Chemie International Edition 49(45), pp. 8480-8483. 2010. DOI: 10.1002/anie.201004169.

[4] B. K. Bose. Global warming: Energy, environmental pollution, and the impact of power electronics. IEEE Industrial Electronics Magazine 4(1), pp. 6-17. 2010. DOI: 10.1109/MIE.2010.935860.

[5] R. B. Finkelman. Health impacts of coal: Facts and fallacies. Ambi AMBIO: A Journal of the Human Environment 36(1), pp. 103-106. 2007.

[6] L. Fan and C. Zhu, Principles of Gas-Solid Flows. Cambridge University Press, 1998.

[7] G. Gronald and J. J. Derksen. Simulating turbulent swirling flow in a gas cyclone: A comparison of various modeling approaches. Powder Technology 205(1-3), pp. 160-171. 2011. DOI: http://dx.doi.org/10.1016/j.powtec.2010.09.007.

[8] B. Wang, D.L. Xu, K.W. Chu and A.B. Yu,. Numerical study of gas-solid flow in a cyclone separator. Appl. Math. Model. 30(11), pp. 1326-1342. 2006. DOI:

http://dx.doi.org/10.1016/j.apm.2006.03.011.

[9] A. Yilmaz and E. K. Levy. Formation and dispersion of ropes in pneumatic conveying. Powder Technology 114(1-3), pp. 168-185. 2001. Available: http://dx.doi.org/10.1016/s00325910 (00)00319-3. DOI: 10.1016/s0032-5910(00)00319-3.

[10] J. Mathieu and J. Scott, An Introduction to Turbulent Flow. Cambridge University Press, 2000.

[11] Rodi, Wolfgang,. DNS and LES of some engineering flows, Proceedings of the Numerical Simulation of Turbulent Flow Research Commemorative Symposium 35th Seiken Symposium Tokyo Japan 20030623-20030624, FLUDYN Fluid Dynamics Research 38(2-3), pp. 145-173. 2006.

[12] J. Fröhlich and D. von Terzi. Hybrid LES/RANS methods for the simulation of turbulent flows. Prog. Aerospace Sci. 44(5), pp. 349-377. 2008. DOI:

http://dx.doi.org/10.1016/j.paerosci.2008.05.001. 
[13] Y. Zhiyin. Large-eddy simulation: Past, present and the future. Chinese Journal of Aeronautics 28(1), pp. 11-24. 2015. DOI: http://dx.doi.org/10.1016/j.cja.2014.12.007.

[14] V. Yakhot, S.A. Orszag, S. Thangam, T.B. Gatski and C.G. Speziale,. Development of turbulence models for shear flows by a double expansion technique. Physics of Fluids A: Fluid Dynamics 4(7), pp. 1510-1520. 1992. Available: http://dx.doi.org/10.1063/1.858424. DOI: $10.1063 / 1.858424$.

[15] K. A. Ibrahim, M.A. El-Kadi, M.H. Hamed and S.M. El-Behery,. Numerical simulation of gas-solid two-phase flow in curved duct, in Energy: Production, Distribution and Conservation, Milan, 2006.

[16] J. Smagorinsky. General circulation experiments with the primitive equations. Mon. Weather Rev. 91(3), pp. 99-164. 1963. Available: http://dx.doi.org/10.1175/1520-0493 (1963)0912.3.co; 2. DOI: 10.1175/1520-0493(1963)0912.3.co; 2.

[17] Ansys Inc., Ansys Fluent Theory Guide. Ansys Inc., 2009.

[18] A. Yilmaz and E. K. Levy. Roping phenomena in pulverized coal conveying lines. Powder Technology 95(1), pp. 43-48. 1998. Available: http://dx.doi.org/10.1016/s0032-5910 (97)033147. DOI: $10.1016 / \mathrm{s} 0032-5910(97) 03314-7$.

[19] H. Akilli, E. K. Levy and B. Sahin. Gas-solid flow behavior in a horizontal pipe after a $90 \hat{\mathrm{A}}^{\circ}$ vertical-to-horizontal elbow. Powder Technology 116(1), pp. 43-52. 2001. Available: http://dx.doi.org/10.1016/s0032-5910 (00)00360-0. DOI: 10.1016/s0032-5910(00)00360-0.

[20] N. Huber and M. Sommerfeld. Characterization of the cross-sectional particle concentration distribution in pneumatic conveying systems. Powder Technology 79(3), pp. 191-210. 1994. Available: http://dx.doi.org/10.1016/0032-5910 (94)02823-0. DOI: 10.1016/00325910(94)02823-0.

[21] Y. Kliafas and M. Holt. LDV measurements of a turbulent air-solid two-phase flow in a 90ï ${ }^{1 / 2}$ bend. Exp. Fluids 5(2), pp. 73-85. 1987. Available: http://dx.doi.org/10.1007/bf00776177. DOI: $10.1007 / \mathrm{bf} 00776177$.

[22] W. Yang and B. Kuan. Experimental investigation of dilute turbulent particulate flow inside a curved bend. Chemical Engineering Science 61(11), pp. 3593-3601. 2006. Available: http://dx.doi.org/10.1016/j.ces.2006.01.013. DOI: 10.1016/j.ces.2006.01.013.

[23] D. O. Njobuenwu, M. Fairweather and J. Yao. Prediction of turbulent gas-solid flow in a duct with a $90 \hat{\mathrm{A}}^{\circ}$ bend using an eulerian-lagrangian approach. AIChE J. 58(1), pp. 14-30. 2011. Available: http://dx.doi.org/10.1002/aic.12572. DOI: 10.1002/aic.12572.

[24] H. Kruggel-Emden and T. Oschmann. Numerical study of rope formation and dispersion of non-spherical particles during pneumatic conveying in a pipe bend. Powder Technology 268pp. 219-236. 2014. Available: http://dx.doi.org/10.1016/j.powtec.2014.08.033. DOI: 10.1016/j.powtec.2014.08.033. 
[25] S. S. Guda, S. L. Rowan and I. Celik, "Rope formation for gas solid flow in a 90 degree bend with varying particle size distributions," in 10th International Conference on Heat Transfer, Fluid Mechanics and Thermodynamics, Orlando, Florida, 2015.

[26] J. McMillan, F. Shaffer, B.Gopalan, J.W. Chew, C. Hrenya, R. Hays, S.B. Reddy Karri and R. Cocco,. Particle cluster dynamics during fluidization. Chemical Engineering Science 100pp. 39-51. 2013. Available: http://dx.doi.org/10.1016/j.ces.2013.02.047. DOI: 10.1016/j.ces.2013.02.047.

[27] R. Cocco, F. Shaffer, R. Hays, S.B. Reddy karri and T. Knowlton,. Particle clusters in and above fluidized beds. Powder Technology 203(1), pp. 3-11. 2010. Available: http://dx.doi.org/10.1016/j.powtec.2010.03.023. DOI: 10.1016/j.powtec.2010.03.023.

[28] S. Gokaltun, J. Varona, A. Awwad and D. Roelant,. Detection of particle clustering in gas solid systems using shadow-sizing technique. Powder Technology 220, pp. 98-103. 2012. Available: http://dx.doi.org/10.1016/j.powtec.2011.09.022. DOI: 10.1016/j.powtec.2011.09.022.

[29] X. An, M. Liu and Y. Fu. Clustering behavior of solid particles in two-dimensional liquid solid fluidized-beds. China Particuology 5(5), pp. 305-311. 2007. Available: http://dx.doi.org/10.1016/j.cpart.2007.07.001. DOI: 10.1016/j.cpart.2007.07.001.

[30] L. C. Gomez and F. E. Milioli, "Numerical Simulation of Fluid Flow in CFB Risers - a Turbulence Analysis Approach Turbulence parameters," Journal of the Braz. Soc. of Mech. Sci \& Eng., vol. XXVII, pp. 141-149, 2005.

[31] S. Vaishali and R. Shantanu. Numerical simulation of gas-solid dynamics in a circulating fluidized-bed riser with geldart group B particles. Industrial Engineering Chemistry Research 46(25), pp. 8620-8628. 2007.

[32] S. Benyahia, H. Arastoopour and T. M. Knowlton. Two-dimensional transient numerical simulation of solids and gas flow in the riser section of a circulating fluidized bed. Chem. Eng. Commun. 189(4), pp. 510-527. 2002. Available: http://dx.doi.org/10.1080/00986440212093. DOI: $10.1080 / 00986440212093$.

[33] Y. Tsuji, T. Tanaka and S. Yonemura. Cluster patterns in circulating fluidized beds predicted by numerical simulation (discrete particle model versus two-fluid model). Powder Technol 95(3), pp. 254-264. 1998. Available: http://dx.doi.org/10.1016/s0032-5910 (97)03349-4. DOI: $10.1016 / \mathrm{s} 0032-5910(97) 03349-4$.

[34] H. Zhou, G. Flamant, D. Gauthier and J. Lu,. Numerical simulation of the turbulent GasParticle flow in a fluidized bed by an LES-DPM model. Chem. Eng. Res. Design 82(7), pp. 918926. 2004. Available: http://dx.doi.org/10.1205/0263876041596788. DOI: $10.1205 / 0263876041596788$.

[35] T. Li, S. Pannala and M. Shahnam. CFD simulations of circulating fluidized bed risers, part II, evaluation of differences between 2D and 3D simulations. Powder Technology 254pp. 115- 
124. 2014. Available: http://dx.doi.org/10.1016/j.powtec.2014.01.022. DOI:

10.1016/j.powtec.2014.01.022.

[36] J. Capecelatro, P. Pepiot and O. Desjardins. Numerical characterization and modeling of particle clustering in wall-bounded vertical risers. Chem. Eng. J. 245pp. 295-310. 2014. Available: http://dx.doi.org/10.1016/j.cej.2014.02.040. DOI: 10.1016/j.cej.2014.02.040.

[37] S.S. Guda, S. L. Rowan, T. Yang, F. Shaffer and I.B. Celik, Investigation of Rope Formation in Gas-Solid Flows through a $90^{\circ}$ Pipe Bend Using High Speed Videos and CFD Simulations, (accepted and being published in the Journal of Computational Multiphase Flows) .

[38] Vester,A.K, Sattarzadeh, S.S and Ramis,O. Combined hot-wire and PIV measurements of a swirling turbulent flow at the exit of a $90 \hat{\mathrm{A}}^{\circ}$ pipe bend. Journal of Visualization 19(2), pp. 261273. 2016. Available: http://dx.doi.org/10.1007/s12650-015-0310-1. DOI: 10.1007/s12650-015$0310-1$.

[39] Chen, X. and Wang, J., "A Comparison of two-fluid model, dense discrete particle model and CFD-DEM method for modeling impinging gas-solid flows," Powder Technology, vol. 254, pp. 94-102, 2014.

[40] F. Rutten, M. Meinke and W. Schroder, "Large-eddy simulations of $90^{\circ}$ pipe bend flows," Journal of Turbulence, vol. 2, pp. 1-14, 2001.

[41] M. J. Tunstall and J. K. Harvey, "On the effect of a sharp bend in a fully developed turbulent pipe flow," Journal of Fluid Mechanics, vol. 34, pp. 595-608, 1968.

[42] F. Shaffer, H. Massah, J. Sinclair and M. Shahnam,. Measurement of time-averaged particle-wall collision properties using particle tracking velocimetry, CRADA PC93-006, final report. Office of Scientific and Technical Information (OSTI). 1995 Available:

http://dx.doi.org/10.2172/3715. DOI: 10.2172/3715.

[43] L. Cabezas-Gómez, RC. da Silva, H.A. Navarro and F. Eduardo,. Cluster identification and characterization in the riser of a circulating fluidized bed from numerical simulation results. Appl. Math. Model. 32(3), pp. 327-340. 2008. DOI:

http://dx.doi.org/10.1016/j.apm.2006.12.005.

[44] M. Syamlal, W. Rogers and T. J. O'Brien, "MFIX documentation: Theory guide," U.S. Department of Energy, Office of Fossil Energy, Morgantown Energy Technology Center, 1993.

[45] F. Mathey, D. Cokljat, J. P. Bertoglio and E. Sergent,. "Specification of LES inlet boundary condition using vortex method," in 4th International Symposium on Turbulence, Heat and Mass Transfer, Antalya, Turkey, 2003.

[46] M.Target, LP AMINA, Private Communication, China, 2014-2016. 


\section{Appendix A}

\section{Numerical Error Propagation}

MFIX simulations have been performed to study the behavior of numerical errors that might get introduced on account of differences in time averaging period. A two-dimensional fluidized bed has been simulated and the effect of individual variation in several parameters has been studied.

\section{Geometry and Boundary Conditions}

A 2D rectangular fluidized bed with a width of $16 \mathrm{~cm}$ and length of $120 \mathrm{~cm}$ has been used as shown in Figure A.1. The bed height is $50 \mathrm{~cm}$ with an initial void fraction value of 0.9 . The cases considered have 36 cells in the X-direction and 135 cells in the Y-direction.

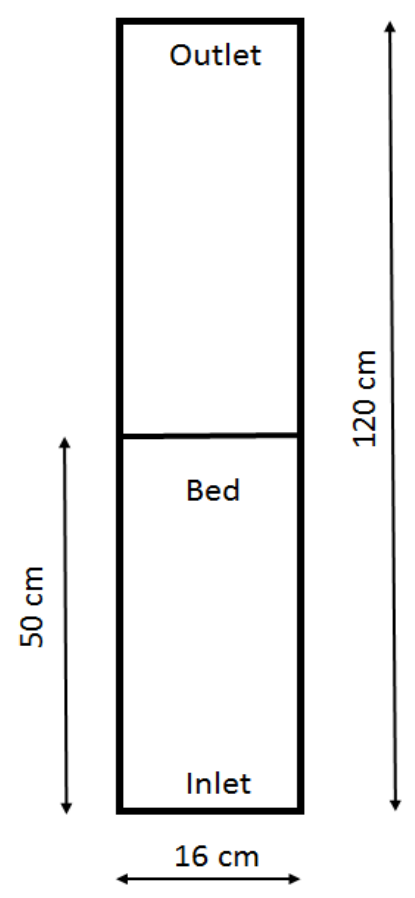

Figure A.1 Geometry of the fluidized bed 
Gas is introduced uniformly at the inlet with a velocity of $180 \mathrm{~cm} / \mathrm{s}$. The outlet is a pressure outlet. The density of the solids in the bed is $2 \mathrm{~g} / \mathrm{cc}$ and the particles have a uniform diameter of $0.04 \mathrm{~cm}$. The walls on both the sides of the inlet are no-slip boundaries. The time step used is 1.0e-04.

\section{Results}

The residual used is $1.0 \mathrm{e}-03$. The number of iterations per time step is 1000 . The total simulation duration is $120 \mathrm{~s}$. Time averaging has been performed for different periods starting from simulation time of $40 \mathrm{~s}$ to avoid the effect of initial transients. Time averaging has been performed for periods of $20 \mathrm{~s}, 40 \mathrm{~s}, 60 \mathrm{~s}$ and $80 \mathrm{~s}$. The time averaged mean gas-velocity profiles at various heights are shown in Figure A.2. It can be clearly seen that there are appreciable differences in velocity profiles at all the locations indicating the occurrence of error due to different time averaging periods.
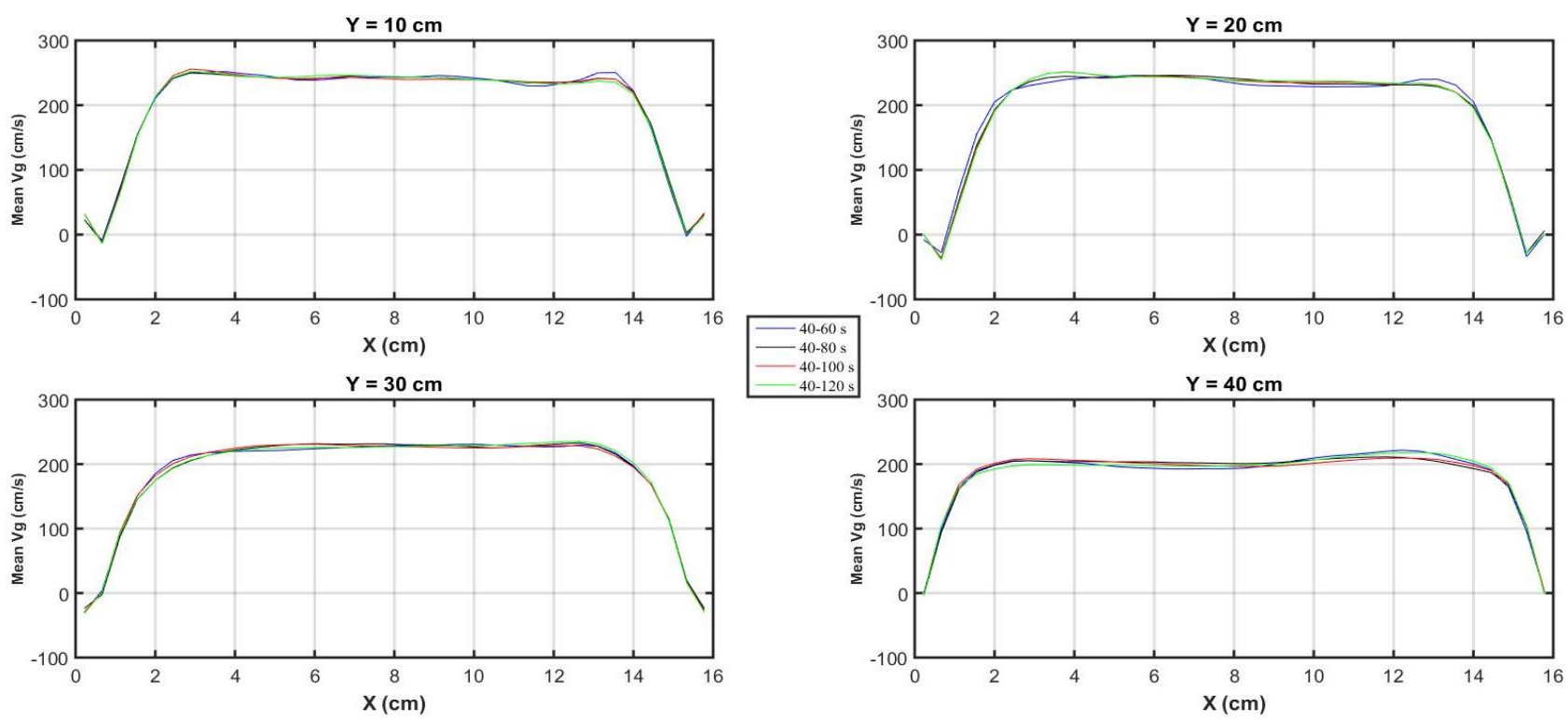

Figure A.2: Time averaged mean gas velocity profiles for different time averaging periods at various heights of the fluidized bed. 
In order to evaluate the error, the case with time averaging period of $80 \mathrm{~s}$ has been taken as the reference case and the absolute error has been calculated for the other time averaging period cases as the difference from the results of 80 s. The time averaged mean absolute error for the gas velocity profiles at all the earlier locations is shown in Figure A.3.
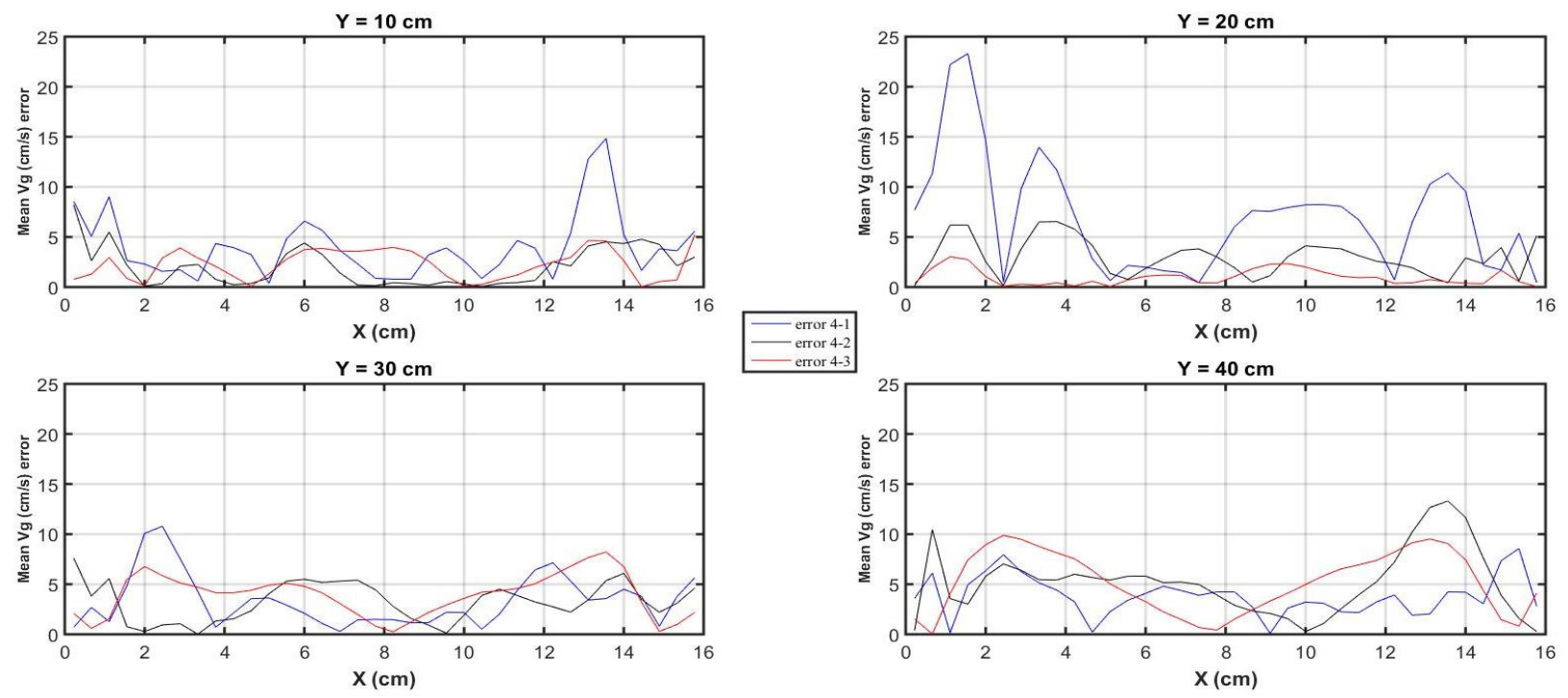

Figure A.3 Estimated time averaged mean gas velocity error profiles for different time averaging periods at various heights of the fluidized bed.

It is seen that the overall error is largest for the least time averaged period and decreases as the time averaging period increases at almost all the locations. Figure A.4 shows the time averaged mean absolute error for the solids velocity profiles at all the earlier locations. The error pattern is similar to that of the gas velocity error profiles. The clusters move from wall to wall in time. Based on the duration of averaging, it is likely that the clusters can be oriented to either side of the wall. Hence the asymmetry in the profiles for shorter time averaging periods. 

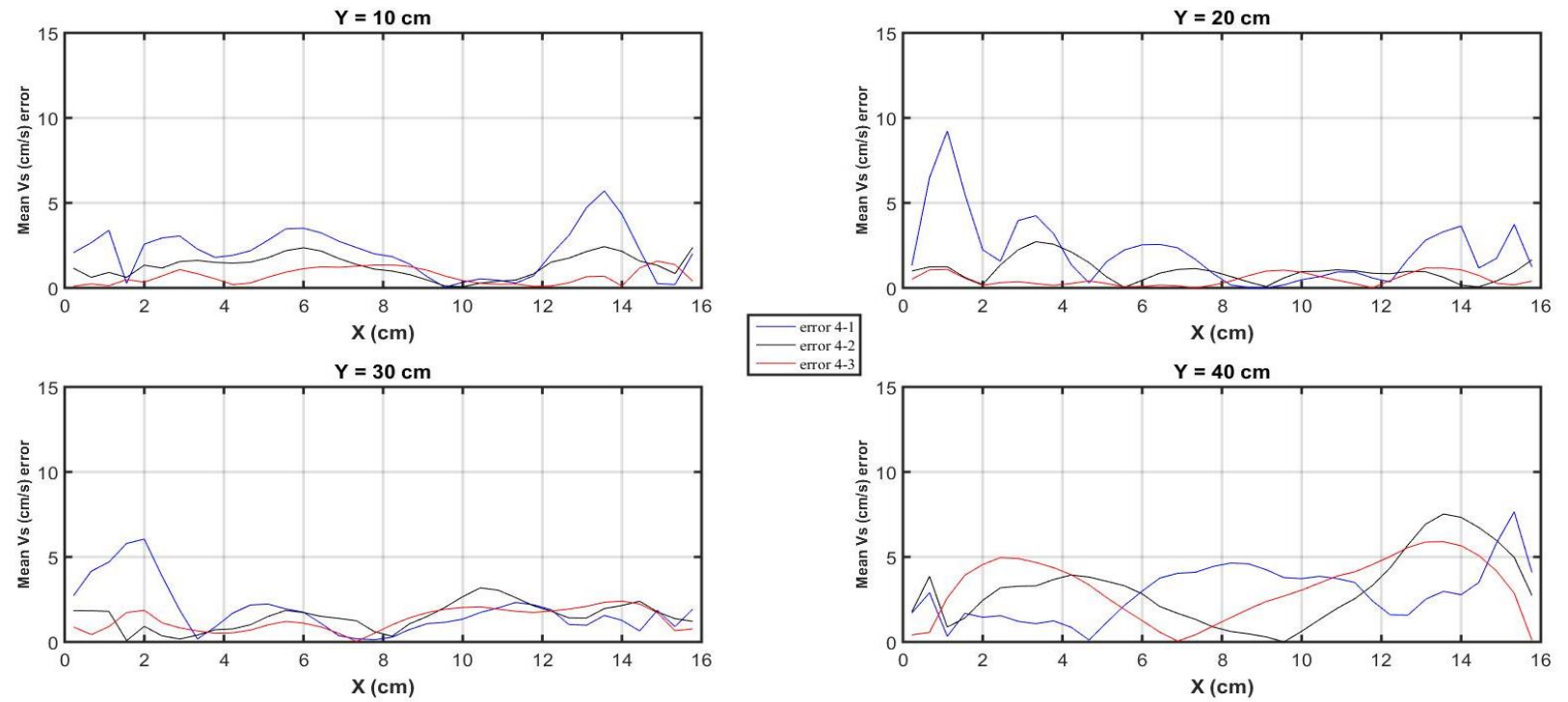

Figure A.4 Estimated time averaged mean solid velocity error profiles for different time averaging periods at various heights of the fluidized bed. 